\title{
Studying the Evolution of Bitcoin-related Topics Extracted from an Online Forum
}

\author{
by
}

\section{Davoud Saljoughi Badlou}

\author{
A Thesis submitted to \\ the Faculty of Graduate Studies and Research \\ in partial fulfilment of \\ the requirements for the degree of \\ Master of Computer Science
}

Department of Computer Science

Carleton University

Ottawa, Ontario, Canada

January 2021

Copyright (c)

2021 - Davoud Saljoughi Badlou 
The undersigned recommend to

the Faculty of Graduate Studies and Research

acceptance of the Thesis

\title{
Studying the Evolution of Bitcoin-related Topics Extracted from an Online Forum
}

\author{
Submitted by Davoud Saljoughi Badlou \\ in partial fulfilment of the requirements for the degree of \\ Master of Computer Science
}

Dr. Olga Baysal, Thesis Supervisor

Dr. Ahmed El-Roby, Internal Examiner

Dr. Mohamed Al Guindy, External Examiner

Dr. Matthew Holden, Chair of Defence

Dr. Michel Barbeau, Department Chair

Carleton University

2021 


\section{Abstract}

Besides all uncommon events of 2020, Bitcoin finally passed 20,000 USD in this year, and grabbed more attention about what is going on cryptocurrency and what will happen next? So far, several researchers used social media data in their works and the role of public opinion especially in the specialized forums on Bitcoin price was proved. To have a better understanding about the discussions in a Bitcoin specialized forums, in this thesis we studied the evolution of discussions in bitcointalk.org, as the main references of discussions related to Bitcoin over the past 10 years. We found 31 different topics in the forum by applying LDA topic modeling and categorized them into 6 categories: Future, General, Mining, Monetary, Regulations, and Technical. Our investigation on this work finds relation between Bitcoin price and the sentiment polarity of selected categories of discussions in the forum. 


\section{Acknowledgments}

I, Davoud Saljoughi Badlou, would like to thank my supervisor, Professor Olga Baysal, this work could not be finished during this special time in 2020 without her complete support. She was always present, from day one to the end of this project, and I will never forget the way she helped me during this thesis, and I hope to have the chance in the future to work with her.

I also thank Professor Mohamed Al Guindy for his advice and guidance on finding the research questions for this research project and also suggesting to work on Bitcointalk as the data source.

I also thank CUFS RTA for providing an award for this work as the funding has allowed me to concentrate my efforts in completing this work.

Further, I would like to acknowledge the help of Hema Sri Kambhampati, my classmate from COMP5117, for participating in the manual labeling process of this work, without her help and time the results would not be as reliable as they are.

I am also grateful for Mahnaz Kamran's input, she assisted me by proofreading this thesis, and her work has improved the quality of this thesis.

Last but not least, I would like to thank my parents, Hayedeh and Ahmad, my siblings Leila and Pourya for their endless love and support in my entire life. 


\section{Table of Contents}

Abstract

Acknowledgments $\quad$ iv

$\begin{array}{ll}\text { Table of Contents } & \text { v }\end{array}$

List of Tables $\quad$ ix

List of Figures $\quad$ xi

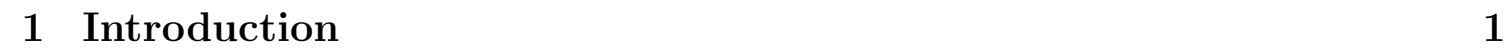

1.1 Public Opinion in Cryptocurrency Studies . . . . . . . . . . . . . . . 1

1.2 Motivation . . . . . . . . . . . . . . . . . 2

1.3 Research Questions . . . . . . . . . . . . . . . . . . 3

1.4 Contributions . . . . . . . . . . . . . . . . . . . . . . . 6

1.5 Organization of the Thesis $\ldots \ldots \ldots$. . . . . . . . . . . . . . . . 7

\begin{tabular}{|lll|}
\hline 2 & Background and Related Work & 8 \\
\hline
\end{tabular}

2.1 Cryptocurrency and Bitcoin . . . . . . . . . . . . . . . . . . . 8

$2.1 .1 \quad$ Story of Bitcoin . . . . . . . . . . . . . . . . 8

2.1 .2 Blockchain Technology . . . . . . . . . . . . . . . . . . 9

$2.1 .3 \quad$ Cryptocurrencies Applications . . . . . . . . . . . . . 11

$2.1 .4 \quad$ Bitcoin Price and Cryptocurrencies Valuation . . . . . . . . . 12 
2.1 .5 Public Opinion effects on Cryptocurrencies . . . . . . . . . . . 13

2.2 Topic Modeling . . . . . . . . . . . . . . . . . . . . . . . 14

2.2 .1 Latent Dirichlet Allocation . . . . . . . . . . . . . . . . . . . 15

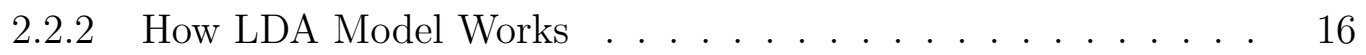

2.2 .3 How many topics a good LDA model should have? . . . . . . 17

2.2 .4 Validation of LDA models . . . . . . . . . . . . . . . . . . . . 19

2.2 .5 Text Preprocessing for LDA . . . . . . . . . . . . . . . . . 20

2.2 .6 Topic modeling vs. self-organizing map . . . . . . . . . . . . . 22

2.3 Related Work . . . . . . . . . . . . . . . . . . . . . . . . . . . . . . 24

$2.3 .1 \quad$ Application of Topic Modeling . . . . . . . . . . . . . . . . . . 24

2.3 .2 Cryptocurrency Analysis in Social Media . . . . . . . . . . . . 26

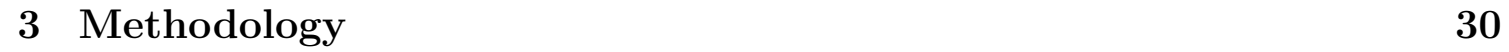

3.1 Datasets . . . . . . . . . . . . . . . . . . . . . . . 30

3.1 .1 Bitcointalk Data . . . . . . . . . . . . . . . . . . 31

3.1 .2 Bitcoin Price

3.2 Data Modelling and Processing Techniques . . . . . . . . . . . . . . . 32

3.2 .1 Data Preprocessing . . . . . . . . . . . . . . . . . . 32

3.2 .2 Evaluating LDA models . . . . . . . . . . . . . . . . . . . . . 34

3.2 .3 Training Models . . . . . . . . . . . . . . . . . . . . . . . 37

3.2 .4 Manual topic labeling. . . . . . . . . . . . . . . . . . . 40

3.2 .5 Sentiment Analysis . . . . . . . . . . . . . . . . . . . . . . 41

3.2 .6 Data Analysis . . . . . . . . . . . . . . . . . . . . . . 42

3.3 Tools and Libraries Used . . . . . . . . . . . . . . . . . . . . . . . . . 44

4 Analysis and Results 45

4.1 RQ1: What are the discussions in a Bitcoin specialized forum about? 45

4.2 RQ2: How do the discussion topics on the forum evolve over the time? 51 
4.2 .1 Future . . . . . . . . . . . . . . . . . . . . . . . . . . . . 53

4.2 .2 General . . . . . . . . . . . . . . . . . . . . . . 53

4.2 .3 Mining . . . . . . . . . . . . . . . . . . . . . 54

4.2 .4 Monetary . . . . . . . . . . . . . . . . . . 55

4.2 .5 Regulations $\ldots \ldots \ldots \ldots \ldots \ldots \ldots$

4.2 .6 Technical $\ldots \ldots \ldots \ldots \ldots$

4.3 RQ3: How do the number of posts in each topic change over the time? 60

4.3 .1 Future . . . . . . . . . . . . . . . . . . . . . . . . . . 61

4.3 .2 General $\ldots \ldots \ldots \ldots$. . . . . . . . . . . . . . . . . . 61

4.3 .3 Mining . . . . . . . . . . . . . . . . . . . . 65

4.3 .4 Monetary . . . . . . . . . . . . . . . . . . . 65

4.3 .5 Regulations . . . . . . . . . . . . . . . . . . . . 74

4.3 .6 Technical . . . . . . . . . . . . . . . . 76

4.4 RQ4: What is the sentiment polarity of discussions in each topic? . . 81

4.4 .1 Future . . . . . . . . . . . . . . . . . . . . . . . . . . 82

4.4 .2 General $\ldots \ldots \ldots \ldots$

$4.4 .3 \quad$ Mining $\ldots \ldots \ldots \ldots$

4.4 .4 Monetary . . . . . . . . . . . . . . . . . . 86

4.4 .5 Regulations $\ldots \ldots \ldots \ldots$

4.4 .6 Technical $\ldots \ldots \ldots \ldots$

$4.5 \quad$ RQ5: Is there any correlation between the evolution of discussion topics related to Bitcoin and the change in Bitcoin prices? . . . . . . . . . 92

\begin{tabular}{|lll}
\hline 5 Discussion & 95 \\
\hline
\end{tabular}

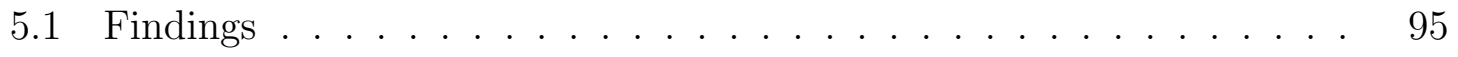

$5.2 \quad$ Implications $\ldots \ldots \ldots \ldots \ldots$

$5.2 .1 \quad$ Implications for Bitcoin pricing analysts $\ldots \ldots \ldots . \ldots 99$ 
5.2 .2 Implications for Bitcoin community . . . . . . . . . . 100

5.3 Threats to Validity $\ldots \ldots \ldots \ldots \ldots$

\begin{tabular}{lll}
\hline 6 & Conclusions & 103
\end{tabular}

6.1 Summary of Contributions . . . . . . . . . . . . . . . . . 103

6.2 Future Work . . . . . . . . . . . . . . . . . . . . . . . . 104

\begin{tabular}{ll}
\hline List of References & 106
\end{tabular}

\begin{tabular}{|l|l|}
\hline Appendix A Sentiment Polarity Analysis Results & 115
\end{tabular} 


\section{List of Tables}

$1 \quad$ Coherence score of topic modeling with different K. . . . . . . . . . . 35

2 Coherence score of Mallet implementation of topic modeling with different K. . . . . . . . . . . . . . . . . . . . . . . . . . . . 35

$3 \quad$ Number of posts and coherence score for each set . . . . . . . . . . . 38

4 Sentiment compound score threshold. . . . . . . . . . . . . . . . . . . 42

5 Map of topics. . . . . . . . . . . . . . . . . . . 46

$6 \quad$ Frequent words in each topic. . . . . . . . . . . . . . . . . . . . . 47

$7 \quad$ Number of posts and frequency of topics in each category. . . . . . . 52

$8 \quad$ Datasets used in previous research. . . . . . . . . . . . . . . . 98

$9 \quad$ Distribution of positive/negative posts in categories/subcategories over the time. . . . . . . . . . . . . . . . . . . . . . 116

10 Distribution of positive/negative posts in Future category topics over the time. . . . . . . . . . . . . . . . . . . . . . 116

11 Distribution of positive/negative posts in General category topics over the time. . . . . . . . . . . . . . . . . . . 117

12 Distribution of positive/negative posts in Mining category topics over the time. . . . . . . . . . . . . . . . . . . . . . 117

13 Distribution of positive/negative posts in Monetary category topics over the time. . . . . . . . . . . . . . . . . . . . . . . . . . . . . . 118 
14 Distribution of positive/negative posts in Regulations category topics over the time. . . . . . . . . . . . . . . . . . . . . . . . . . . . . 118

15 Distribution of positive/negative posts in Technical category topics over the time . . . . . . . . . . . . . . . . . . . 119 


\section{List of Figures}

1 Research roadmap. . . . . . . . . . . . . . . . . . . . . 4

$2 \quad$ A graphical representation of the distribution of topics in a document

in an LDA model $[1] . . \ldots \ldots$. . . . . . . . . . . . . 16

$3 \quad$ An overall scheme of parameter assignment in a LDA model. . . . . . . 18

4 pyLDAvis visual with 10 topics. . . . . . . . . . . . . . . . . 36

5 pyLDAvis visual with 15 topics. . . . . . . . . . . . . . . . . 37

$6 \quad$ Sample summary table for 2017 a dataset. . . . . . . . . . . . . . . . . 39

7 Number of posts in categories. . . . . . . . . . . . . . . . . . . . . . . 50

$8 \quad$ Presence of the topics in each categories for each dataset. . . . . . . . 53

$9 \quad$ Topics over the time in Future category. . . . . . . . . . . . . . . . . 53

10 Topics over the time in General category. . . . . . . . . . . . . . . . . 54

11 Topics over the time in Mining category. . . . . . . . . . . . . . . . . 55

12 Topics in Monetary category. . . . . . . . . . . . . . . . . . . . . . . 55

13 Topics over the time in Currency subcategory. . . . . . . . . . . . . . 56

14 Topics over the time in Financial subcategory. . . . . . . . . . . . . . 57

15 Topics over the time in Price subcategory. . . . . . . . . . . . . . . . 57

$16 \quad$ Topics over the time in Regulations category. . . . . . . . . . . . . . . 58

17 Topics in Technical category. . . . . . . . . . . . . . . . . . . . . . . . 58

18 Topics over the time in Wallet subcategory. . . . . . . . . . . . . . . 59

$19 \quad$ Topics over the time in Others subcategory. . . . . . . . . . . . . . . 59 
20 Total number of posts over the time. . . . . . . . . . . . . . . 60

$21 \quad$ Number of posts in categories over the time. . . . . . . . . . . . . . 62

22 Percentage of categories for discussions over the time. . . . . . . . . . 63

23 Discussion topics over the time in Future category. . . . . . . . . . . . 64

24 Distribution of topics over the time in Future category. . . . . . . . . 64

25 Discussion topics over the time in General category. . . . . . . . . . . 66

26 Distribution of topics over the time in General category. . . . . . . . 67

27 Discussion topics over the time in Mining category. . . . . . . . . . . 68

28 Distribution of topics over the time in Mining category. . . . . . . . . 68

$29 \quad$ Discussion topics over the time in Monetary subcategory. . . . . . . . 69

$30 \quad$ Distribution of topics over the time in Monetary subcategory. . . . . 70

$31 \quad$ Discussion topics over the time in Currency subcategory. . . . . . . . 71

32 Distribution of topics over the time in Currency subcategory. . . . . . 71

33 Discussion topics over the time in Financial subcategory. . . . . . . . 72

34 Distribution of topics over the time in Financial subcategory. . . . . 72

35 Discussion topics over the time in Price subcategory. . . . . . . . . . 73

$36 \quad$ Distribution of topics over the time in Price subcategory. . . . . . . . 74

37 Discussion topics over the time in Regulations category. . . . . . . . . 75

38 Distribution of topics over the time in Regulations category. . . . . . 75

39 Discussion topics over the time in Technical subcategories. . . . . . . 76

$40 \quad$ Distribution of topics over the time in Technical subcategories. . . . . $\quad 77$

$41 \quad$ Discussion topics over the time in Wallet subcategory. . . . . . . . . 78

$42 \quad$ Distribution of topics over the time in Wallet subcategory. . . . . . . 78

43 Discussion topics over the time in Others subcategory. . . . . . . . . 79

44 Distribution of topics over the time in Others subcategory. . . . . . . 80

45 Average sentiment compound score of the total posts over the time. . 81

$46 \quad$ Distribution of positive/negative posts over the time. . . . . . . . . 82 
47 Compound score of posts in categories over the time. . . . . . . . . 83

48 Compound score of topics in Future category over the time. . . . . . 84

49 Distribution of positive/negative posts in Future category over the time. 84

$50 \quad$ Compound score of topics in General category over the time. . . . . . 85

51 Distribution of positive/negative posts in General category over the time. . . . . . . . . . . . . . . . . . . . 85

52 Compound score of topics in Mining category over the time. . . . . . 86

53 Distribution of positive/negative posts in Mining category over the time. 87

54 Compound score of subcategories in Monetary category over the time. 87

55 Distribution of positive/negative posts in Monetary category over the time. . . . . . . . . . . . . . . . . . . . . . 88

56 Compound score of topics in Monetary category over the time. . . . . 88

57 Compound score of topics in Regulations category over the time. . . . 89

58 Distribution of positive/negative posts in Regulations category over the time. . . . . . . . . . . . . . . . . . . . . . . . . . . . . . 89

59 Compound score of subcategories in Technical category over the time. 90

60 Compound score of topics in Technical category over the time. . . . . 91

61 Distribution of positive/negative posts in Technical category over the time. . . . . . . . . . . . . . . . . . . . . 91

62 Overall correlation of Bitcoin price with parameters. . . . . . . . . . . 93

63 Correlation of Bitcoin price with topic category discussion volume, sentiment and percent of discussions per topic category. . . . . . . . . 93 


\section{Chapter 1}

\section{Introduction}

In this chapter, Section 1.1 opens describing the idea of this thesis and highlighting the role of public opinion in cryptocurrency studies. Section 1.2 presents our motivation for this work. Section 1.3 outlines our research questions, while Section 1.4 highlights the key contributions. Section 1.5 describes the organization of the thesis.

\subsection{Public Opinion in Cryptocurrency Studies}

Cryptocurrencies have emerged in the past decade as alternate forms of exchanging value [2]. The demand for cryptocurrencies has been increasing rapidly in the world, as they are accepted and used for many different types of payment and trading [3]. Cryptocurrencies, from the earliest years of their birth, were different from Fiat Currencies and have some distinct differences with traditional currencies. They are decentralized, and their distributed nature has resulted in the fact that up until now, almost none of these cryptocurrencies has been supported by a central government or international institution. Their ownership is anonymous as it is not possible to track the real ownership of an asset, and they are mainly traded online.

Based on the state-of-the-art research literature, the price of cryptocurrencies is volatile and fluctuates more than the traditional monetary items [3, 4]. The price of 
Bitcoin, the largest cryptocurrency by market capitalization [5], has been fluctuating from less than 1,000 USD at the beginning of 2017 to more than 19,000 USD in less than nine months. But it has dropped to around 6,000 USD in the middle of 2018 and continues to fluctuate since [6]. Even, at the time of writing this thesis, in December 2020, it has reached again 19,000 USD and is highly likely to hit 20,000 USD before the end of this year. As we describe in Section 2.1.4, cryptocurrencies' valuation, analyzing their price volatility and analysing their prices is an open research question, and numerous research studies have been conducted in this area [7].

Social media data is one of the important sources of information for analyzing the cryptocurrencies' price [8]. Public opinion in the Bitcoin-specialized forums such as Bitcointalk.org or Reddit.com has been used for analyzing the impact of users' discussions on Bitcoin's price in recent papers [6] 8$]$. In this work, we will investigate the data extracted from social media within a long period of time (past 10 years) to gain a comprehensive view about this domain and the role social media plays in the world of cryptocurrency. In order to do that, first we determine discussion topics from the online forum and study the topic evolution over time. Then, we apply the sentiment analysis on the posts and determine how sentiment evolves over the time. Finally, we explore the correlation between the discussion topics and their sentiment and the price of Bitcoin.

\subsection{Motivation}

With the increasing prevalence of Bitcoin in trades, a growing number of people participate in online discussions on forums to share their knowledge. Bitcoin is usually traded online, and it is the common practice for the traders to make their trading decisions based on the information they gain on the online discussion forums [9]. Users of these forums and websites tend to share their opinions, reasoning and concerns 
which in a long term create a rich source of information.

The previous research in this area has already recognised the importance of public opinion in future price of Bitcoin and involved this factor in their price analysis models. However, to the best of our knowledge, virtually almost all of them have considered the effect of social media content as a static factor and because of the change in the public opinion about a topic, these models tend to lose their accuracy over time. The aim of this research is to study and analyze the changes in the discussion topics and see how these topics evolve over time. We are interested in highlighting the changes that happened in the past to show the effect of the changes in discussions on the traditional predictive models.

Cryptocurrency, in general, is a new term and it takes a lot of time and effort to be well understood by people. Considering this fact, in this research we study discussions and posts in online forums to provide a new insight about Bitcoin. We collect and categorize the online debates, interpret them and link them to the Bitcoin price fluctuation. The result of this work can be used in cryptocurrencies' price analysis. Moreover, the final findings of our work can be helpful for gaining a better perception about different aspects of this domain.

\subsection{Research Questions}

The goal of this thesis is to gain new insights about Bitcoin by mining and investigating bitcointalk.org. Figure 1 illustrates the road map that we followed to provide answers to the following research questions:

\section{RQ1: What topics are discussed in a Bitcoin specialized forum? On} bitcointalk.org, users who are interested in cryptocurrency share their ideas and seek feedback from others. Over the past decade, many different topics have been discussed in this forum. In RQ1, we detect and classify all the discussed 
Data Crawling from Bitcointalk.org

560 k posts between Dec 2009 - Oct 2020

\section{Data Pre Processing}

\section{Implementing LDA Topic Modeling}

\section{Sentiment Analysis}
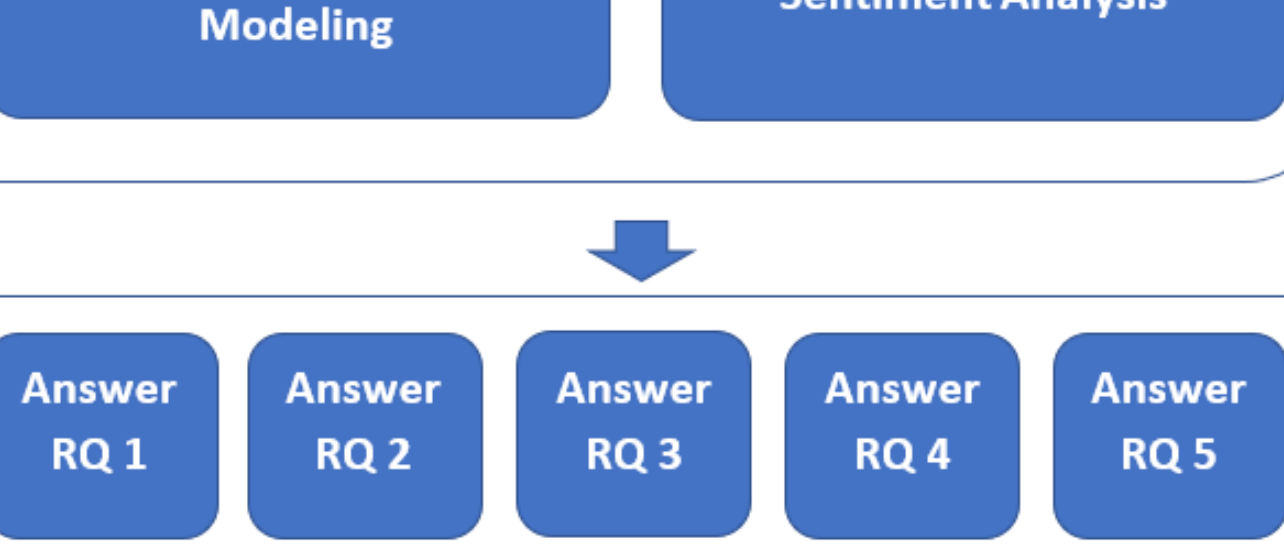

\section{Answer \\ RQ 5}

Figure 1: Research roadmap. 
topics in this forum. This classification provides comprehensive metadata about the discussions in the cryptocurrency domain and helps researchers to have a better understanding about the domain and its discussion topics.

\section{RQ2: How do the discussion topics on the forum evolve over the} time? The social media data affects cryptocurrency price, and it is used as an input factor in cryptocurrency's price analysis. In this research question, we study the patterns of the topics, when the new topic emerges and how long it lasts. These findings highlight the evolution patterns of the topics in the forum. The result of this research question can help the designer of current models to consider the evolution of topics in their models and reach higher performance.

3. RQ3: How does the topic's volume of posts change over time? As we mentioned in the previous RQ, some topics are used in the price analysis models. In this research question, we study the changes in the number of posts over time. The result of this RQ can offer new insights into the discussion trends of the topics over time. We can gain insights into what topics are heavily discussed during the past decade. The results can be also useful for tuning the price analysis models as the change in the discussion topics could be taken as an effective input parameter in the model.

4. RQ4: What sentiment is associated with each discussion topic? The sentiment is measured as sentiment polarity of the posts in the forum, and can be used as a feature to be added to the model for analyzing the future price of cryptocurrencies. Previous research has considered sentiment as a factor in their price analysis models. In this research question, we analyze the sentiment similarity between different discussion topics. We try to find how different discussion topics in the forum are similar in terms of their sentiment polarity, as well as the way these polarities change over the time. It's important to 
understand what topics carry a more positive or negative user opinion. The results of this RQ can highlight the difference in sentiment across various topics, and lays the foundation for the researchers to study sentiment in different topics. To the best of our knowledge, most works that use sentiment analysis only analyze the sentiment polarity of the full data set, yet presenting the difference of sentiment weights in different topics makes it possible to design more accurate models.

5. RQ5: Is there a correlation between the evolution of the Bitcoinrelated discussion topics and the Bitcoin price volatility? In previous research questions we try to determine factors related to the discussions in the

forum. For the final research question, we try to identify the correlation between Bitcoin's price and these factors. The goal of this RQ is to identify what factors have relation with Bitcoin price. This work explores and highlights the major criteria which can help experts design better price analysis models by focusing on determining factors. Furthermore, this study introduces a number of parameters that may affect Bitcoin price. This, in turn, helps different stakeholders within the Bitcoin community pay attention to these parameters and be informed on the determining factors for making better long-term goals and plans.

\subsection{Contributions}

The contributions of this research study are as follows:

1. A longitudinal analysis of the discussion topics extracted from the online forum during the 10-year time period. 
2. Extraction of 31 topics related to various discussions about cryptocurrency classified across 6 categories.

3. Analysis of the evolution of topics in the forum.

4. Sentiment analysis of the discussion and the study of sentiment changes over the past 10 years.

5. Analysis of the factors related to Bitcoin price.

6. A dataset of the discussions on the Bitcointalk forum.

\subsection{Organization of the Thesis}

The structure of the remaining chapters in this thesis is as follow: Chapter 2 offers background and presents key concepts related to Bitcoin. It also introduces topic modeling, as well as reviews relevant related work in this area. Chapter 3 presents methodology and describes data collection methods and data analysis techniques used in this work. Chapter 4 reports the results and answers to our research questions, while Chapter 5 discusses implications of our work. And finally, Chapter 6 concludes this thesis by offering the summary and discussion of future directions for this line of research. 


\section{Chapter 2}

\section{Background and Related Work}

In this chapter, we first provide background on cryptocurrency and Bitcoin in Section 2.1. We then present topic modelling in Section 2.2. Finally, in Section 2.3, we discuss the previous works related to our research and highlight the novelty of our work in this domain.

\subsection{Cryptocurrency and Bitcoin}

The goal of this section is to describe the role that our results can play in the cryptocurrency domain. We start the item with the "Story of Bitcoin" in Section 2.1.1 and then continue the text with a brief review on blockchain technology and its applications in Section 2.1.2 and Section 2.1.3. Then, we discuss the valuation of cryptocurrencies in Section 2.1.4 and discuss the effects of public opinion on cryptocurrencies in Section 2.1.5,

\subsubsection{Story of Bitcoin}

The story of Bitcoin began with a paper titled "Bitcoin: A Peer-To-Peer Electronic Cash System" published by a person or group named Satoushi Nakomoto, the identity is yet to be confirmed, in 2008. The paper described a method of direct online payment 
without the need for any financial institution to be involved in the process. Some months later on January 2009, an open source program which implemented this new protocol was released and the story of Bitcoin began. As the leading cryptocurrency, Bitcoin, has had its ups and downs over the past decade. Playing a major role in Silk road black market, rising its value by nearly 2000 percent in 2017 are among the most mentionable points in the history of this popular cryptocurrency. Right now with over 400 billion CAD, Bitcoin owns nearly 60 percent of the whole cryptocurrencies market values.

As the main focus of this study will be on the discussions on social media, we reviewed some of the previous works and prerequisites for it. We will start with a brief description of blockchain as the main technology behind Bitcoin and other cryptocurrencies and describe the way they work. In the later sections, we present applications of cryptocurrencies and continue with the valuation of Bitcoin and the effects of public opinion on it.

\subsubsection{Blockchain Technology}

Like all other digital currencies, Bitcoin is not saved on any specific database, and the records of all activities are stored on a blockchain [3]. A blockchain is a distributed database of public ledger/records that have been executed and shared among different participating parties [10]. Generally, users of a system are required to be authenticated in order to insert a new record into the database. Similarly, any new piece of information or transaction needs to be validated by the majority of the peer-topeer network to be saved in a blockchain. Blockchain uses public and private keys for verification of information and saves the data in an encrypted format. Like all other cryptocurrencies, Bitcoin uses "blockchain technology", we explain this technology on the example of Bitcoin transactions. Assume Alan wants to transfer one bitcoin to Bob in his "Wallet". Alan prepares a new block with the "public key" of 
Bob (receiver) and his own "private key" (sender) and broadcasts it to the network. The network validates the transactions by checking two criteria: first of all it checks whether the sender account actually belongs to Alan by checking his digital signature, and secondly, it verifies whether he has sufficient bitcoins in his account by reviewing his previous transitions. If these steps are passed, this transaction will be saved as a new chain in the ongoing blockchain, and from that time on, Bob will be considered as the owner of the transferred bitcoin.

We now provide the definitions of the terms used in the above example [11]:

- Wallet: A Bitcoin (cryptocurrency) wallet on the Bitcoin network is similar to the physical wallet in daily life. It involves "private key", which is the element that enables users to make a transaction in the blockchain. A wallet shows the total balance and enables users to pay some bitcoins to any other member in the network.

- Blockchain: is a record of all previous records for the cryptocurrency and is publicly available. Blockchain verifies the validity of a transaction, and also is used for preventing "double spending".

- Double-Spending: if one user tries to spend one bitcoin for two different payments simultaneously, it is called double-spending, and is stopped with the help of mining in a blockchain.

- Mining: each new transaction needs to be verified in the network, and cryptocurrencies use mathematical calculation to do it. That is, only the miner, who solves the latest problem, is allowed to add the block to the blockchain. For instance, in the above example, the first miner who solves the problem will be able to transfer the ownership information of the transferred bitcoin from Alan to Bob and will earn some bitcoins as a reward of its work. 
In conclusion, blockchain plays the role of a trustworthy intermediary party like banks in traditional transactions, and provides a reliable environment with a peer to peer (P2P) network. Given the fact that the data is decentralized and distributed in different nods on a network, it is safeguarded against being stolen or lost, like what it used to be in the traditional databases. Furthermore, since the responsibility of storing and processing data is distributed among some parties, it is highly unlikely to be dependent on any central authority or government. Having said that, there is only one special and hypothetical case that might threaten a cryptocurrency which is known as 51 percent attack. This attack happens when a number of miners gain the control of more than 51 percent of the network, thereby being able to apply any change they want in the blockchain. The blockchain technology has revolutionized the world of finance and opened new doors to state-of-the-art applications without the need for any governmental or central unit to be involved in the process. We present some of its most important applications next.

\subsubsection{Cryptocurrencies Applications}

Blockchain has laid the foundation for many services to be delivered in a decentralized manner. The range of these services is so wide that many specialists such as professor Tapscott called the technology as "blockchain revolution" [3]. Cryptocurrencies have numerous applications and in this section we review six distinct categories of them suggested by McKinsey \& Company [12].

1. Static registry: it is a distributed database for storing reference data which are the information that enable users to have an estimation of ownership's properties.

2. Identity: the next is related to storing personally identifiable information which can be used in elections or identification processes. 
3. Smart contracts: smart contracts are self-executing and automatic transactions which occur between buyer and seller based on a decentralized consensus record 13$]$.

4. Dynamic registry: for keeping records of the current owner of an asset, i.e., a shipping material in supply chain or stocks.

5. Payment infrastructure: the way people can transfer money without the need for any third party to transfer the money, either locally or internationally.

6. Others: there are many other applications. For example, by using smart contracts, many IoT devices are able to directly trade between themselves. As an illustration, a traffic camera can earn the cost of its electricity bills by selling some information to traffic monitoring apps.

\subsubsection{Bitcoin Price and Cryptocurrencies Valuation}

One of the most controversial questions in the world of cryptocurrencies has been about the intrinsic value of Bitcoin and the possible ways to calculate it. Finding the ways to predict the future value of Bitcoin and the determining factors has been quite interesting and even crucial for different stakeholders [9]. Investors, for instance, require predictive models to plan for their investments and calculate the expected return. Policymakers, on the other hand, need some means by which they can take proper measures to minimize financial risks and increase stability in the world of cryptocurrencies [14]. The price of Bitcoin at the time of its launch was less than 1 USD, and about one decade after its launch time, this value reached 20,000 USD. The price of this currency has experienced many fluctuations, and doubling or losing half of its value during only one season has been quite common. Only at the time of writing this thesis, the price of Bitcoin fluctuated between 7,500 and 23,000 USD [15]. 
There must be far more explanations for Bitcoin price fluctuation and this phenomena cannot be sufficiently explained by standard macroeconomic theories $7,16,19$

Finding a proper answer to this question along with analysing Bitcoin price and predicting its future value is a challenge in the world of economics and many papers have targeted this domain. Given the wide range of these researches, we only mention [20], [21], [22], and [23] as few examples among hundreds of works. One of the matters about evaluation of Bitcoin price that has attracted attention in many papers is the effect of social media on Bitcoin price. Not only the change in price causes many debates in social media, but some discussions result in change in the price of Bitcoin. We will discuss this matter in Subsection 2.1.5 with more details.

\subsubsection{Public Opinion effects on Cryptocurrencies}

A variety of stakeholders are involved in cryptocurrency communities such as traders, miners, users, developers and so on. There are a number of platforms that provide the possibility of discussing cryptocurrencies. The most familiar forums for these discussions are official forums such as bitcointalk.org and reddit.com, as well as social media such as Facebook and Twitter [24]. From the past, a number of research studies have been carried out in this domain to determine whether social media can affect the price of Bitcoin and reveal their relation [9]. Some studies have adopted Wikipedia and Google Trend in public opinion analysis, for instance, Kristoufek et al. 25] and [18] surveyed the correlation between Bitcoin price and the information on the above mentioned examples, or Jin et al. [26], worked in the Google Trends data to predict $\mathrm{ICO}^{1}$. Several other researchers have adopted Wikipedia and Google Trend in their works [25], 27], 6].

The other source of data that has been used in public opinion analysis widely is Twitter. Twitter posts have been the source of data for many stock analysis papers

\footnotetext{
${ }^{1}$ Initial Coin Offering
} 
such as 28 and 29. Given the similarity of Bitcoin and stocks as an asset, Twitter has been widely adopted in the Bitcoin price analysis. For instance, the sentiment weight of tweets has been used to analyze Bitcoin price [30], and [31], [32].

Another adoption in the Bitcoin price analysis is the use of the information on specialized forums, and the two major sources in these works are Reddit and Bitcointalk [8], [6], [7].

Existing state-of-the-art research highlights the key role of social media in Bitcoin price prediction. Moreover, posts in forums usually affect Bitcoin price more strongly compared to tweets [33]. Based on this evidence, in our research we focus on specialized forums that are dedicated to the Bitcoin discussions and try to answer the questions related to the Bitcoin discussion and social sentiment (as presented in Section 1.3). In order to become more familiar with the existing research literature, we study the state-of-the-art and discuss previous works in Section 2.3.2.

\subsection{Topic Modeling}

Topic models are a modern class of text analysis methods that have been embraced by a range of scholars in the social sciences, humanities and beyond. The most peculiar feature of topic models is that they remove the need for a supervisor to provide initial insights for the model to be trained. In other words, topic models facilitate an automated procedure for coding the content of a corpus of texts into a set of substantively meaningful coding categories called "topics" 34.

What made topics models unique among previous models is that they minimize the need for an intelligent agent (like humans) to be involved in the process which caused a revolution in a number of branches of science [35]. Unlike traditional approaches in text processing such as BOW [36], topic models take into account the semantic context of words in their unsupervised training steps [37,38]. 
In this section, we describe Latent Dirichlet Allocation (LDA) in Section 2.2.1 and explain how it works in Section 2.2.2. Section 2.2.3 highlights the good number of topics in an LDA model, while Section 2.2.4 explains its validation. Section 2.2.5 describes key text pre-processing techniques for LDA, and finally, Section 2.2.6 discusses the reason why we selected topic modeling rather than self-organizing map technique.

\subsubsection{Latent Dirichlet Allocation}

The origin of LDA dates back to the 1980's when there was a need for a means to retrieve text information more accurately. The oldest member of the topic modeling family is called Latent Semantic Analysis (LSA) which was introduced by Deerwester, Dumais, Furnas, Landauer, and Harshman [39]. LSA enabled researchers to cluster documents with similar words considering the theory that words with similar semantic ranges are highly likely to be seen together. Several years later, in 2003, Blei, Ng, Jordan, and Lafferty adopted the main idea of LSA and developed another algorithm for topic modeling called Latent Dirichlet Allocation (LDA) [40]. LDA is a generative probabilistic model for a collection of discrete data, for instance, a collection of documents. The basic idea behind this model is that documents of a corpus are represented as random mixtures over latent topics [40]. A topic in this definition is considered to be a distribution over words. This gained a lot of attention and inspired many researchers and academics in their works. LDA represents topics by word probabilities. The words with the highest probabilities in each topic usually illustrate the main concept of the topic. For example, a topic consisting of the words such as "Bitcoin", "digital currencies", "blockchain", and "wallet" that have the highest probability is more likely be labeled as "cryptocurrencies". 

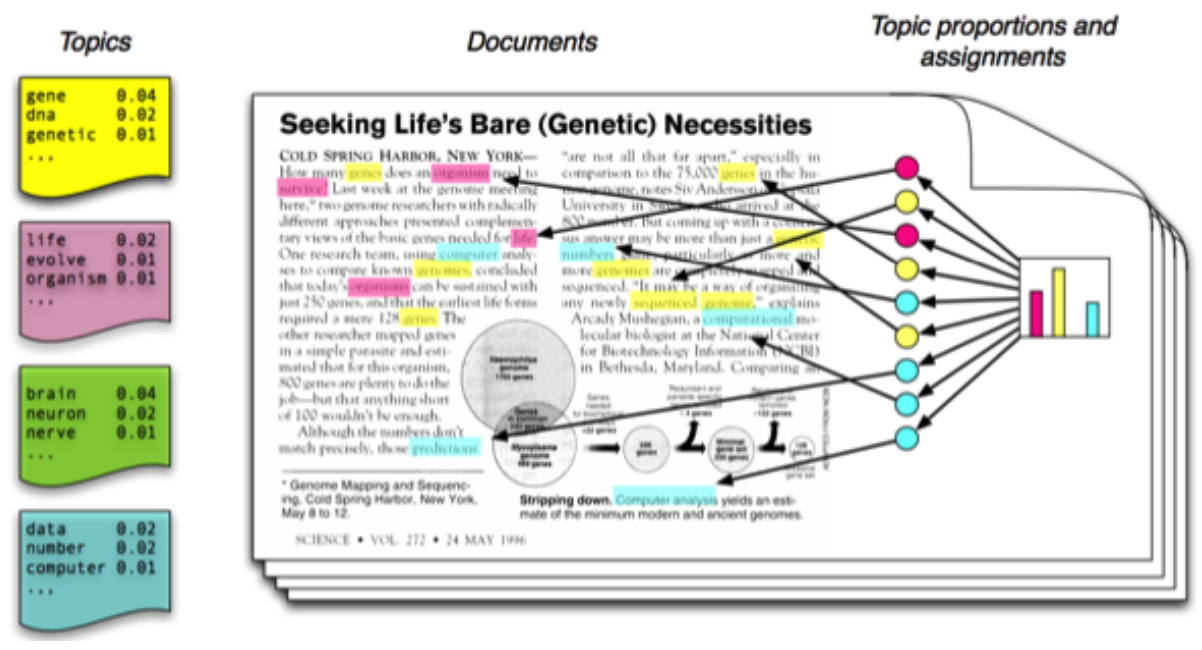

Figure 2: A graphical representation of the distribution of topics in a document in an LDA model [1].

\subsubsection{How LDA Model Works}

LDA follows a generative approach to find the parameters which are used in the model. The primary assumption is that there are $\mathrm{k}$ latent topics according to which documents are generated, and that every topic is represented as a multinomial distribution over the $|\mathrm{v}|$ words in the vocabulary. A random document is created by sampling a mixture of the above-mentioned topics and then sampling words from that mixture [40]. After training a LDA model on a set of documents, every document could be represented as a distribution over topics, but the point is that the probability of topics varies in different documents based on their main semantic context, Figure 2 represents a sample graphical distribution of topics in a document. In order to be trained on a collection of data, LDA models require four fundamental parameters:

1. $\alpha$ : indicates the document's prior distribution. This parameter is generated from a Dirichlet allocation distribution on a random base and with $\alpha$ hyper-parameter.

2. $\beta$ : indicates the topic's prior word distribution. This parameter is generated from a Dirichlet distribution on a random base and with $\beta$ hyper-parameter.

3. $\mathrm{k}$ : indicates the number of topics that we expect the model give us after 
training phase.

4. The number of iterations which specifies the maximum number of runs that the model is allowed to pass to converge.

In a LDA model a document which consists of $\mathrm{N}$ words $\mathrm{w}=\left(w_{1}, \ldots, w_{n}\right)$ is generated according to the following steps:

First, the model makes a sample for $\theta$ from a Dirichlet $\left(\alpha_{1}, \ldots, \alpha_{k}\right)$ distribution. Thus, we can say that $\theta$ lies in the $(\mathrm{k}-1)$ dimensional simplex: $\theta_{i} \geq 0, \sum_{i} \theta_{i}=1$.

In the next step, for every single word among $\mathrm{N}$ words in the corpus, a topic $\mathbf{z}_{n} \in\{1, \ldots, k\}$ is sampled from a $\operatorname{Mult}(\theta)$ distribution $p\left(\mathbf{z}_{n}=i \mid \theta\right)=\theta_{i}$.

Finally, each word $w_{n}$ is sampled, conditioned on the $\mathbf{z}_{n}$ th topic, from the multinomial distribution $P\left(w \mid z_{n}\right)$

According to the above definitions we can write the equation for a document's probability in Formula 1:

$$
p(w)=\int_{\theta}\left(\prod_{n=1}^{N} \sum_{z_{n}=1}^{k} p\left(w_{n} \mid \mathbf{z}_{n} ; \beta\right) p\left(\mathbf{z}_{n} \mid \theta\right) p(\theta ; \alpha) d \theta\right.
$$

In the above equation $P(\theta ; \alpha)$ indicates Dirichlet, $p\left(z_{n} \mid \theta\right)$ is a multinomial parameterized by $\theta$, and $P\left(w_{n} \mid z_{n} ; \beta\right)$ is a multinomial over the vocabulary of the corpus [41. Figure 3 shows an overall schematic of these parameter assignment.

\subsubsection{How many topics a good LDA model should have?}

In order to have concrete topics in a topic model, it is of great importance to choose the right number of topics according to the nature of the corpus. Some academics take this as one of the shortcomings of topic modeling as determining the number of topics is usually a challenging task [43]. While other researchers believe that the right number of topics should be guessed by evaluating the real performance of the model [44]. That is why most of the researchers empirically try several numbers of 


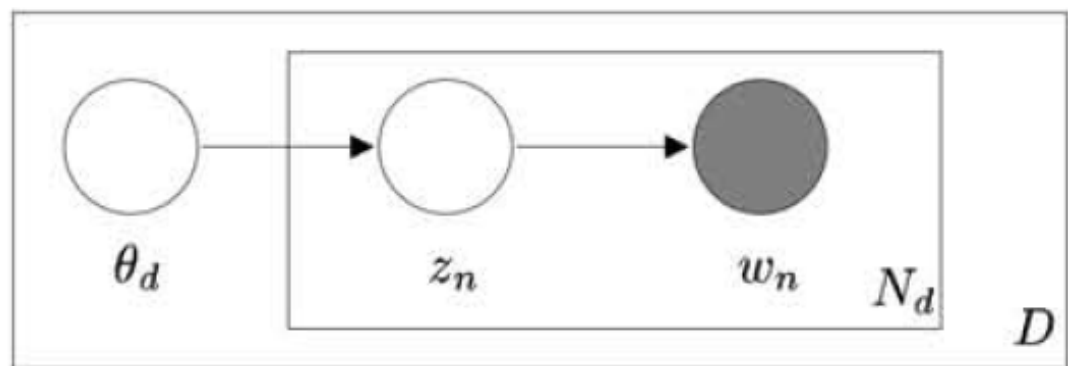

Figure 3: An overall scheme of parameter assignment in a LDA model. The boxes are plates indicating replicates. The outer plate indicates documents in the corpus, while the inner plate is an indicator for the repeated choice of topics and words in a document 42$]$.

topics to determine the best number of topics that suits their data. Carron-Arthur et al [45] examined the performance of the topic model by trying different number of topics known as k. The number of topics varied between 10 and 100 as they monitored the performance of the model by investigating the top words in every topic. They concluded that models containing more than 30 topics tended toward duplication. On the other hand, having less than 20 topics did not lead to satisfactory results as some independent and distinct topics merged together. Yau et al. [43] tested a number of different values for $\mathrm{k}$ ranging from 50 to 200 but ended up using only 50 since interpreting the results can be handled easier and more effectively. These studies do not explicitly indicate how one should choose the number of topics when training a topic model. However, it can be concluded that several tries and errors are required to find the optimum number of the topics. Furthermore, we can say that the right number of topics for a model should strike a balance between topics which are too general (choosing too few topics) and topics which are too specific (choosing too many topics). 


\subsubsection{Validation of LDA models}

One of the most important questions that should be answered after building a topic model on a text corpora is how good the model is? The minimal expectation of an acceptable topic model is that it must have topics which are semantically related. In order to measure coherence level among topics, there are both qualitative and quantitative measures, however, most of the researchers prefer to use qualitative evaluations of the coherence of topics in a topic model [8,46]. For qualitative evaluation, researchers can manually investigate the topics and check if the top words in a topic actually are related and belong to the same context semantically. Martin and Johnson [47] comment that the top words in a topic must not be ambiguous nor be without an interpretable theme. Chang, Boyd-Garber, Gerrish, Wang, and Blei [48] propose a "word intrusion test" to measure the consistency of words in topics. In

their experiment, they selected some topics and replaced one of the top 10 words of these topics with another word calling it "intruder" word. Their judgement about the quality of the model depended on the participant's ability to find this replaced word. If the participant distinguished the intruder word without trouble, the model was considered to be coherent. Carron-Arthur et al. [45] mention the significance of "domain knowledge" in this domain. That is some words might be taken as intruders to a topic by the in-charge participant, while they are actually related to the topic from the specialists in the subject. Researchers have already adopted other methods to validate how precise the topics of a topic model are. Agrawal et al. [49] measured the quality of topics using the words that overlapped in different topics. The overlap metric used was Jaccard similarity. To sum up, we can say that a good topic model must be practical and applicable. A topic model which is useful for gaining an overall insight over a huge corpus of text documents may not be ideal for one which is going to be used for classifying documents precisely. That is why defining strict measures to 
examine the performance of a topic model is not easy nor helpful, and the researchers should decide on some aspect of the model based on their own requirements and expectations.

\subsubsection{Text Preprocessing for LDA}

Pre-processing is a vital step in almost every text processing task including building a topic model. In order to achieve good results and coherent topics, documents should be prepared in a way that the most influential features of the corpus could be extracted and interpreted by the computer [50]. This pre-processing step might include a number of similar tasks such as normalizing the data, tokenizing the documents, removing stop words, stemming, lemmatization, etc.

In normalizing step characters with different written forms will be converted to the same form. For instance "is not" and "isn't" should be considered one word. Furthermore it is of great importance to correct spelling errors in a corpus as much as possible since a machine considers miss-spelled words as nonsense which reduce the quality of the model [51]. Researchers could place a premium on text normalization, especially when the data comes from noisy sources such as social media, text messages and comments on blog posts and web pages, since the use of informal language, abbreviations and misspellings are common in these cases. In a social media corpus removing hashtags, punctuations, users, and other unimportant signs and words forms a great part of the work.

The lemmatization and stemming are two common ways two reduce the number of words in a corpus which belong to the exact same semantic family. For example after lemmatizing a document "see", "saw" and "seeing" would be converted to the same form (i.e., "see" which is the root of the verb). The same rule is adopted in finding the stem of plural words. That is, the word "books" will be converted to "book" after lemmatization [52]. Not only does this reduce the number of words 
which should be processed in a corpus thereby reducing processing time and resource, but the performance of the model is highly likely to increase as the words with the same context are already introduced to be the same. Lemmatization and stemming each normalize the text in helpful ways, but more work may need to be done in this regard. Removing stop words is a standard practice in preparing a corpora for the further processing tasks. The main idea behind this step is that words which are so repetitive and do not have much information could be removed to give the model the opportunity to only concentrate on real informative vocabulary. Furthermore, according to the nature of the corpus, other words may need to be removed as well. For instance, the corpus of scientific journals processed by Suominen and Toivanen 44 tended to have acknowledgment sections which were not informative for their project and they decided to omit these sections in their work. Some of the words that are usually taken as stop words in English documents are "that", "is", "are", etc. In an LDA model, for instance, the existence of such words in a document does not help the model to link the document to the correct topic as they do not have a special meaning.

Apart from removing stop-words which are usually quite repetitive in a corpora, one might decide to remove very rare words in their work as they distract the topic model. In fact, there is not a standard guideline for removing words and researchers usually do apply this based on their experience. Székely and vom Brocke [50] deleted words that appear in fewer than two documents, Carter et al. [53 removed words that exist in fewer than 50 documents or that exist in more than 50 percent of the corpus. Mimno [54] suggests that there could be called a 5-10 rule: remove words that appear fewer than 5-10 times and remove words that appear in more than 5-10 percent of the corpora.

Although these recommendations and experience can give us a good insight, they cannot be applied without paying attention to the objective of the final model. Some 
researchers such as Székely and vom Brocke [50] suggest that in order to make sure that nothing has slipped in the pre-processing step it might be a good idea to check the corpus manually in the final step.

Researchers already have adopted some other techniques to reduce the number of words in a collection of text documents. For example, Martin and Johnson ignored all words except for nouns in their work [4]. They developed three topic models each one based on three different versions of the same news corpus. The first one is the original version of the corpus without any pre-processing, the second is a lemmatized version, and in the third copy of the corpus they only include the lemmatized version which had only noun words. The final result of the experiments revealed that the topic model trained on the third version of the corpus was the most coherent one. In other words, according to their experiment lemmatized nouns are the most informative members of a corpora and omitting other words (verbs, prepositions and adjectives) can help the model to find the semantic relations far easier.

\subsubsection{Topic modeling vs. self-organizing map}

Self-organizing map (SOM) is a type of artificial neural network (ANN) that is trained using unsupervised learning to produce a low-dimensional (typically twodimensional), discretized representation of the input space of the training samples, called a map, and is therefore a method to do dimensionality reduction [55]. A SOM can parse the entire dataset in different groups according to the similarity of the features of input data. SOM is useful for visualization of complex or high dimensional data in a low dimensional map typically with two dimensions [56]. Since the goal of our research is studying the evolution of topics, SOM cannot elaborate on the topics of discussions like LDA. When applying SOM, we would need to find the topic of each cluster. 
In our work, for finding the evolution of topics, we split our dataset into 22 sixmonth periods and ran the LDA model for each period separately. According to Rzeszutek et al. [57], it is possible to apply LDA-SOM for finding the evolution of topics. In this method, researchers initially run topic modeling over the entire dataset and then use SOM (clustering technique) for studying the evolution of topics over the time.

We provide the following justification for the choice of leveraging topic modelling over SOM in this work:

- Since previous works have used topic modeling, we decided to be consistent with the selection of our methods to be able to compare our results and findings with the state-of-the-art research.

- Both methods are simply different machine learning techniques. Our choice of LDA vs. LDA-SOM is subject to the following pros and cons:

\section{- Pros:}

* LDA models are trained for each of the 22 datasets, thus, reducing the time to train each model on a smaller data compared to the entire dataset that SOM is using for training.

* Topic modeling finds similar sets of words across different discussion posts. For example, both CPU and GPU are frequent words in the same "Mining" topic. Since SOM is trained on the entire data, different words would be treated as different topics. However, training LDA on smaller datasets would allow us to put these two words into one topic.

* Parameter optimization is essential for LDA, as well as manual inspection of the topic numbers since some topics only exists in very limited series. It is possible to miss such topics with SOM. 
- Cons:

* Our method includes manual topic labeling. SOM is more automated and can reduce human errors.

* A new LDA-based topic model needs a new customized preprocessing prior to training, while SOM can handle live data for training a model.

According to Section 2.3.2, previous works in this domain used topic modeling and thus, to be consistent with the previous research, we apply topic modeling in our work.

\subsection{Related Work}

We review previous research that adopted topic modeling in Section 2.3.1 and then discuss previous works on social media analysis related to Bitcoin in Section 2.3.2.

\subsubsection{Application of Topic Modeling}

Topic modeling has been a hot topic over the past decade and it has won over many researchers who work on natural language processing tasks. Many articles have been published based on topic modeling approaches in a wide range of subjects such as linguistic science, social network, software engineering, etc. Chen at el. [58 have studied the possible ways by which topic models can play a constructive role in software repositories. They provided a collection of articles which were published between Dec 1999 and Dec 2014. Aiming at finding the works related to software engineering that applied topic models, they identified and demonstrated the research trends in mining unstructured repositories using topic models. They found that many software engineering tasks were neglected and only basic topic models were adopted in these works [34]. 
An important challenge in the natural language processing world is text summarization which takes the most important parts of a document and creates an informative summary from a document automatically. Arora and Ravindran [59] used LDA to find latent variables in word level and SLDA (sentence-based latent Dirichlet allocation) to do the same thing in sentence level. They used Document Understanding Conference (DUC) which is a news corpus in their work and managed to achieve higher precision and recall and F-measure compared to other methods. Zhai et al. 60] aimed at adopting topic modeling in opinion mining with a focus on product reviews. The problem that this work tries to resolve is the wide range of words used in reviews to describe the same feeling. In other words, reviewers express the same opinion with diverse phrases and words which makes the further processing tasks challenging. This work used topic modeling to group semantically related words together. Furthemore, it suggests that pre-existing information can help the topic model to gain better results. Nolasco et al. 61 designed a modular algorithm which works based on topic modeling and introduced a new term called "sub-event". In order to address the problem in previous works (existing models assumed an event to be a single entity ignoring that it can be composed of other events over time), they suggested a model that identifies sub-events and creates labels to represent them more accurately. They evaluated their proposed method using two large-scale Twitter collections. Their approach detected several sub-events, most of them were related to real sub-events. Naskar et al. 62 tried to focus on discussions on social networking websites to find the sentiment behind sentences in online conversations. Their goal was to identify users' feelings in the social network through their conversations. They conducted a study to determine whether users of social networks (Twitter, in particular) have a tendency to form gatherings based on their common interests. Jin et al. [63] introduced Targeted Aspects Oriented Topic Modeling (TATM) to find better-oriented topics in documents where short length of text is a concern. The benefit of their 
proposed strategy is that that the topics which are generated using their model are more focused and homogeneous as each topic has its focus on a specific aspect. After training their model on a real-word database they ended up producing more concrete and complete topics.

\subsubsection{Cryptocurrency Analysis in Social Media}

Social media messages are one of the predictor parameters in Bitcoin valuation 9 and the majority of investors develop their investment strategies on cryptocurrencies considering the resources they find on the Internet 7,64 .

This area has gained increased attention in many research works related to cryptocurrencies. Many researchers focus on quantitative data and search engine information by studying Google trends, Wikipedia, or quantitative statistics of social media, mainly Twitter, in their recent research $9,16,18,31,65,66]$.

Although the content of popular social media such as Twitter has been the main source of analysis in many research works, there are some concerns about the quality of these data [8]. First of all, many researchers put forward the argument that many tweets might be spam thus worthless. And secondly, people talking about cryptocurrencies usually do not discuss other topics, thus, analyzing their opinion on the specialized forum related to Bitcoin may provide better results.

To the best of our knowledge, there are only a limited number of research works that have performed some explorations of the social media that focus on cryptocurrencies such as Www.bitcointalk.org and www.reddit.com. In this section, we review these works in more detail and discuss their findings.

Kim et al. in 2016 [64], tried to provide a predictive model for cryptocurrency using the related discussions about Bitcoin, Ethereum, and Ripple, three cryptocurrencies with big market size and dedicated public discussion forums on that time. They developed a web crawler to collect comments from web forums and tagged 
them using a sentiment analysis tool called VADER. This tool proposed by Hutto et al. can be used for classifying comments in online communities [67]. It assigns all comments with a score in the range -1 to 1 for negative and positive ones.

For the next step, they applied the Granger causality test to find the correlation between the fluctuation in cryptocurrencies' prices and comments in forums. This test has been already adopted in many researches related to market shares and currencies 68. The input data are online comments, the price of cryptocurrencies and the number of transactions of cryptocurrencies. The findings of this paper suggest that user comments and replies can be a source of data for price prediction, there is a relation between positive vs. negative comments on price change but the pattern is different for each cryptocurrency, and there is a positive relationship between the number of transactions and replies in the forums. All in all, this work was one of the earliest researches on the role of public opinion on cryptocurrencies, but it studied a limited history of cryptocurrencies at that time.

Lee et al. [6] have tried to build predictive model with deep learning using the comments in an online forum. They used the comments on www.bitcointalk.org as their main source of input data and scrapped the content of this website between December 2013 and September 2016. They also used Google daily Trends and Wikipedia daily usage for Bitcoin for training of their learning model. For their analysis, they first used Nonnegative matrix factorization (NMF) algorithm of topic modeling for creating an initial lexicon. For the next step, they expanded the corpus using kernel density estimation (KDE). They had selected 10 concepts among items and provided scores for all of them per topic. Finally, they applied a deep learning model based on the above mentioned KDE-based topic scoring and other quantitative data such as the number of topics, the sum of replies, the sum of views, Google Trend Value, and Wikipedia Pageviews. The best prediction result was achieved when they used a three-layer neural network which had an accuracy of more than 80 percent. 
Kang et al. [7] have tried to recognize the influencer groups in social media who mainly discussed Bitcoin. They used the online discussions on www.bitcointalk.org as the input dataset for their paper. They crawled all discussions from November 22, 2009 to February 2, 2018 and categorized the users by applying a hypertext-induced topic selection (HITS) algorithm into two groups: opinion leaders and majority users. The authors used Pareto's principle (proposed by Koch et al. [69]) and considered the top 80 percent of authority scores for classifying these two groups. Opinion leaders formed around 11 percent of the members with an overall 49 percent of the total posts and comments in the dataset, and the remaining 51 percent posts and comments were from the 89 percent remaining members. For analyzing the role of influencer, they analyzed the comments in three different groups such as opinion leaders, majority users, and total users. The paper applied the topic modeling technique (NMF) to analyze the comments between June 1, 2015, to February 1, 2018. The findings of the research group are as follows:

- The majority group mainly focused on price, transaction, and investment while the opinion leaders discussed technical terms in addition to their discussion on financial matters.

- There is a similarity between the topics in the opinion leaders and the total members. So it is possible to evaluate only the topics from the opinion leaders group to analyze the entire community.

Philips et al. 8] have focused on determining the relation between the change in the price of cryptocurrency and the topic of discussions in social networks. This research employed the discussions about Bitcoin and Ethereum in a Reddit forum, in technical and trading subreddit forums for Bitcoin and Ethereum. They first applied a dynamic topic modeling approach (an extension of LDA) to retrieve information from social networking websites and then adopted the Hawks model to find the relation between 
the retrieved topics and cryptocurrency prices. The results of their work introduce a direct relationship between the discussions in social media and the change in the price of cryptocurrencies. They claim that some items could be taken as an indicator for increase or decrease in the price. For instance, items such as risk, investment, or trading can indicate price reduction. Conversely, items such as "substantial price movement" indicates price fluctuation, and "fundamental cryptocurrency value" is a signal for a rise in prices. One main limitation of this research is they used four different subreddit forums, and some of the results were not consistent. Therefore, it is essential to investigate the process using a better dataset to enhance the reliability of the results. 


\section{Chapter 3}

\section{Methodology}

This chapter describes the approach we adopted to extract and analyze the evolution

of topics on social media discussions. In Section 3.1 we describe the datasets we collected and used in this thesis, while Section 3.2 presents the data preprocessing, modelling, and analysis techniques. Section 3.3 reports on the software and tools used in this work.

\subsection{Datasets}

In this work, we extracted and collected the discussion data from bitcointalk.org and gathered the historical price data from coindesk.com and bitcoincharts.com. Since researchers have used Twitter, Reddit and/or Bitcointalk data in their works, we offer several reasons justifying our choice of the data source.

- The goal of this research is to study the evolution of discussions related to Bitcoin over time. Thus, we opted for mining the data from a publicly available specialized forum, rather than Twitter data since historical data for Twitter (i.e., past 10 years) is not freely available.

- Twitter data is typically less formal, contains hashtags, slang, and informal 
words. Since topic modelling is sensitive to the quality of the input data, training a reliable topic model is essential for our work.

- Comparing Bitcointalk and Reddit, researchers have worked with Bitcointalk in the past. To be able to compare our findings with previous research, we have decided to mine Bitcointalk for our longitudinal analysis of the discussions.

\subsubsection{Bitcointalk Data}

Bitcointalk.org is a forum website in which users share their opinions on different topics related to the cryptocurrency. The forum is divided into five different sections: Bitcoin, Economy, Local, Alternate cryptocurrencies, and Other. Each section has its own subsections. In this work, we collected all the discussions of the "Bitcoin" section. We used the BeautifulSoup library of Python and crawled all the 48,819 discussion subject posts and 511,233 replies which were posted between December 16th, 2009 and October 1st, 2020. Apart from the main content of the posts, our dataset includes information about their authors, the date and time of the post, and all the replies which were related to the post. This dataset is made available on GitHub1 for future studies.

\subsubsection{Bitcoin Price}

In this work, we collected the daily price of Bitcoin by downloading the data from coindesk.com This website only contains the data from October 1st, 2013. Since we were analyzing the evolution of discussion topic for a longer period of time, we collected the older daily price data from bitcoincharts.com this website offers a range of exchange prices, we used Mt. Gox Bitcoin Exchange as the base for Bitcoin

\footnotetext{
${ }^{1}$ https://github.com/saljoughi/Evolution-of-topics-of-discussion-on-Bitcointalk.org-

${ }^{2}$ https://www.coindesk.com/price/bitcoin

${ }^{3}$ https://bitcoincharts.com/charts/mtgoxSEK\#tgSzm1g10zm2g25zv
} 
price. Mt. Gox Bitcoin Exchange was one of the most important Bitcoin exchanges which became obsolete in 2014 .

\subsection{Data Modelling and Processing Techniques}

We now describe the step-by-step procedure we followed in this work for answering the research questions. Section 3.2.1 presents our preprocessing, followed by our approach for determining the suitable LDA model in Section 3.2.2. Then, we describe our models and their evaluation in Section 3.2.3. We explain the process of our manual topic labeling and semantic analysis in Section 3.2.4 and Section 3.2.5, respectively. Finally, Section 3.2.6 describes the data analysis techniques used for interpreting the results. The resources and documentations of this work can be found in a GitHub repository ${ }^{4}$ for the future works.

\subsubsection{Data Preprocessing}

Bitcointalk.org offers a wide range of topics on the main page and users can choose any topic to explore, different subjects, as well as the replies to the original subject. For keeping the role of the main title in our analysis, we added the title of each subject to the main post, and for the replies we only used the body of their post to avoid entering noise in our input data with some fixed repeating phrases. We stored all the posts along with the time on which they were published sorted by their publishing date; we excluded the posts prior to 2010. Then, we separated the posts into 6 month long periods, resulting in 22 different datasets.

As mentioned in Section 2.2.5, we took the following steps to preprocess our data:

\section{Data cleaning and tokenization}

\footnotetext{
${ }^{4}$ https://github.com/saljoughi/Evolution-of-topics-of-discussion-on-Bitcointalk.org-
} 
We removed URLs and emails as they provide little information about the context, then we deleted all non-English characters and numbers, and removed all the posts that were shorter than 6 words. Then, we split the text into the sentences and sentences to the words and converted all characters to lowercase form and removed all the punctuation. Finally, we tokenized the corpus using gensim library's simple_preprocess 70$]$.

\section{Building bigram and trigram models}

The main idea behind using n-grams is that they can help text processing models gain an insight about the language structure. Bigram and trigram are the sequence of two and three words that are frequent in use at the same time 71 . We built and incorporated bigrams and trigrams to our input data to improve the model's performance.

\section{Lemmatization and stop word removal}

For removing the stop words, we used the standard stop-word library of Python and added some additional words from our own corpus to it, e.g., "bitcoin", "quote", "subject", "edu" or names of week days and months. Then, we lem-

matized the comments using spaCy [72. As suggested by prior work [8, 47], we only kept nouns and adjectives as input to our LDA model.

\section{Removing short words}

It is common practice to delete short words with a length of three or less characters or repetitive words that appear in more than 50 percent of data points, as well as those with very low frequency. In this work, we only delete the words with a length of less than three characters, because there are many common abbreviations with a length of three characters which are related to cryptocurrencies. In addition, we did not delete any word because of its low frequency. 
Since we were building 22 different corpuses, if we set any limitation for the number of appearance of any word, we would miss the track of evolution of some words in different times.

\subsubsection{Evaluating LDA models}

In order to determine the quality of the model and measure its performance, we used the topic coherence score. Topic coherence itself refers to a set of statements or facts that support each other. In the LDA topic modeling domain, topic coherence measures the semantic interpretability of the topics by considering the similarity of the top $\mathrm{N}$ most repeated words, i.e., 10 frequent words in each topic [73]. Based on the study of Röder et al. [74], a number of coherence metrics can be used for assessing the quality of LDA models such as $C_{V}, C_{P}, C_{U C I}$, and $C_{N P M I}$, with $C_{V}$ performing better than others. Therefore, we have used $C_{V}$ metric as the topic coherence measure in this work.

$C_{V}$ coherence is based on applying four steps [75]: 1) segmentation of data in the pair of words (top $\mathrm{N}$ words for each topic), 2) calculating the probability of occurrence of the word and pair of words in the whole documents, 3) calculating a confirmation measure about how every two words in every topic support each other based on the similarity of all words in that topic, 4) and finally, the $C_{V}$ coherence score is equal to the mean of all values measured in step 3 .

According to Röder et al. [74], $C_{V}$ score is in the range of 0 to 1 , where higher $C_{V}$ score illustrates the better implementation of topic modeling. To the best of our knowledge, no minimum is set for the acceptable coherence score, while previous works report the coherence scores of around 0.5 or higher [75 77].

To determine whether all the words (regardless of their part of speech) should be included in training, we run the model using two different sets of words for training the LDA model: 1) all the words in the corpus, and 2) only nouns and adjectives. 
Table 1: Coherence score of topic modeling with different K.

\begin{tabular}{|c|c|c|}
\hline Number of Topics (K) & All POS Tags & Nouns and Adjective Tags \\
\hline 5 & 0.47 & 0.50 \\
\hline 10 & 0.38 & 0.38 \\
\hline 15 & 0.40 & 0.40 \\
\hline 20 & 0.31 & 0.35 \\
\hline 25 & 0.34 & 0.35 \\
\hline 30 & 0.40 & 0.40 \\
\hline 40 & 0.38 & 0.38 \\
\hline 50 & 0.42 & 0.40 \\
\hline 100 & 0.47 & 0.37 \\
\hline
\end{tabular}

Table 2: Coherence score of Mallet implementation of topic modeling with different K.

\begin{tabular}{|c|c|c|}
\hline Number of Topics (K) & All POS Tags & Nouns and Adjective Tags \\
\hline 5 & 0.60 & 0.59 \\
\hline 10 & 0.60 & 0.60 \\
\hline 15 & 0.58 & 0.60 \\
\hline 20 & 0.55 & 0.57 \\
\hline 25 & 0.55 & 0.54 \\
\hline 30 & 0.53 & 0.52 \\
\hline 40 & 0.48 & 0.50 \\
\hline 50 & 0.47 & 0.48 \\
\hline 100 & 0.40 & 0.40 \\
\hline
\end{tabular}



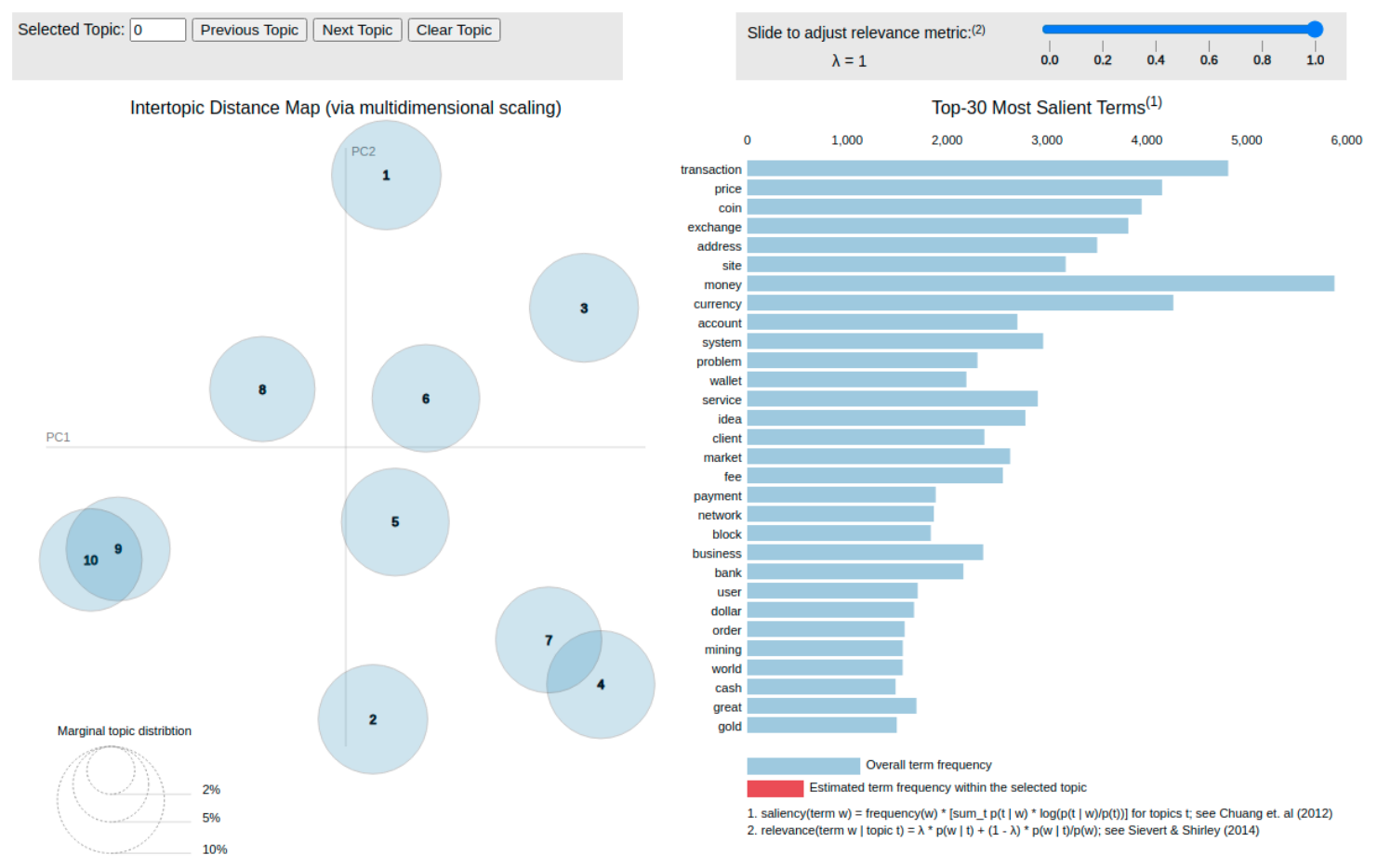

Figure 4: pyLDAvis visual with 10 topics.

As we can see in Table 1, the LDA model produced a better result when we only used nouns and adjectives as the principal components of the posts. In order to investigate an improvement, we applied Mallet implementation to identify whether it can increase the performance of the LDA model. The results are presented in Table2. By comparing Table 1 and Table 2, we can say that using the Mallet implementation can have a positive effect on the coherence score of the generated topics. So we decided to use this implementation in our work.

To determine the right number of topics for our experiments, we trained two LDA Models using Mallet implementation, one with 10 and the other with 15 topics. Moreover, we compared their pyLDAvis visuals to analyze the distribution of topics and the number of occasions on which topics overlap with each other. As shown in Figure 4 and Figure 5, the LDA model produced a better result when we set the number of topics to 15 . 


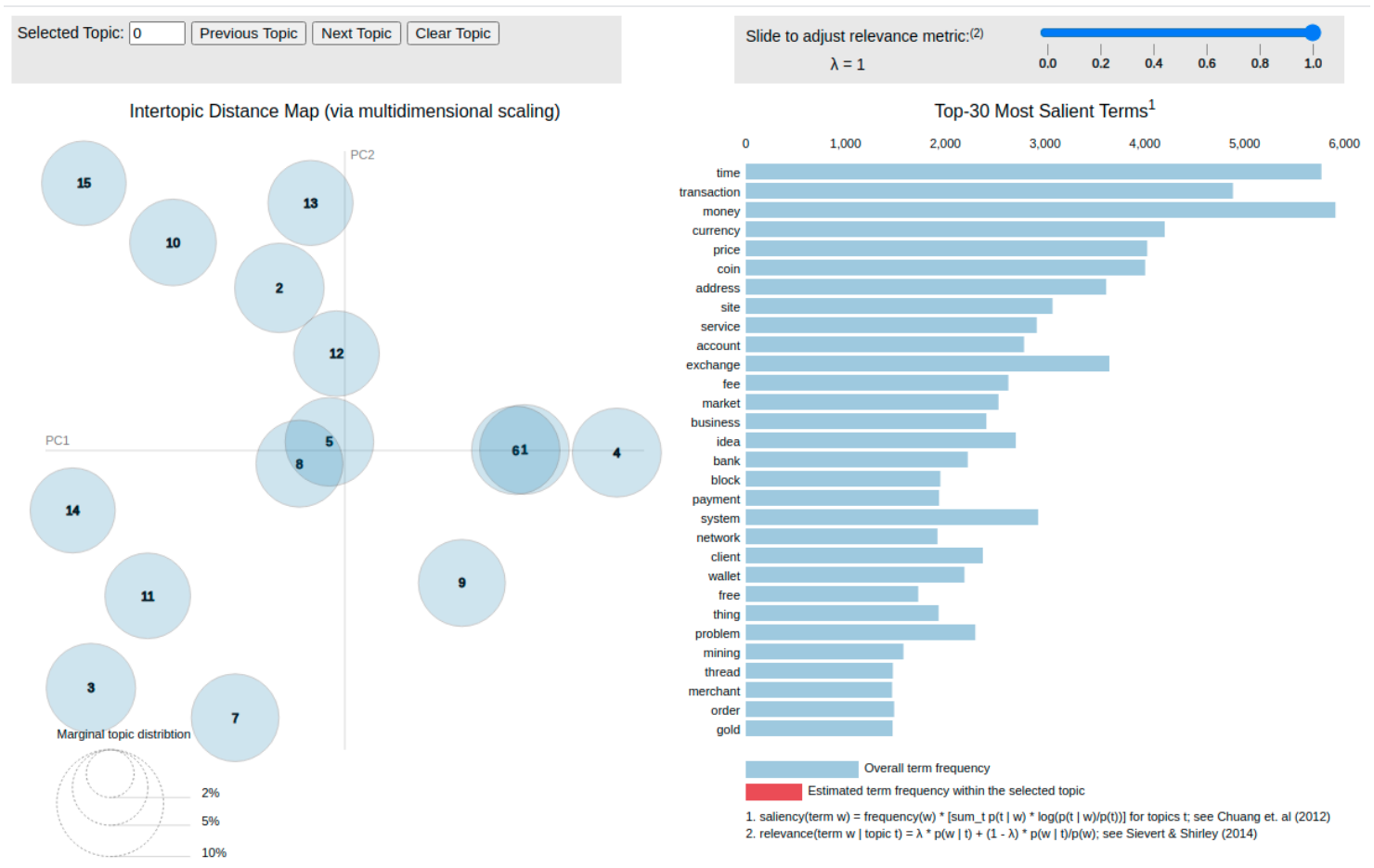

Figure 5: pyLDAvis visual with 15 topics.

\subsubsection{Training Models}

After setting the initialization, we worked with 22 datasets that we had generated for each 6 month period. We trained a separate LDA model for each one of these 22 datasets. Table 3 presents the number of posts in each set and the coherence score of our model on each set.

After training each model, we collected some analytical information about the corpus including the summary table, Pyldavis chart and table of all posts for each dataset.

- Summary table provides valuable information about the distribution of topics in the corpus (the number of posts that are assigned to a topic, the probability of seeing this topic in the whole corpus), as well as the 10 most frequent words and their probability for each topic. A sample of this table is presented in Figure 6. 
Table 3: Number of posts and coherence score for each set

\begin{tabular}{|c|c|c|c|}
\hline Serie & All Posts & Posts (after cleaning) & Coherence Score \\
\hline 2010a & 515 & 495 & 0.45 \\
\hline 2010b & 5,402 & 5,213 & 0.42 \\
\hline 2011a & 32,660 & 30,744 & 0.59 \\
\hline 2011b & 28,848 & 27,341 & 0.59 \\
\hline 2012a & 14,902 & 14,036 & 0.53 \\
\hline 2012b & 12,855 & 12,132 & 0.49 \\
\hline 2013a & 25,327 & 23,744 & 0.54 \\
\hline 2013b & 25,384 & 23,809 & 0.55 \\
\hline 2014a & 46,018 & 43,636 & 0.56 \\
\hline 2014b & 33,100 & 31,624 & 0.56 \\
\hline 2015a & 29,197 & 28,401 & 0.54 \\
\hline 2015b & 27,818 & 27,240 & 0.56 \\
\hline 2016a & 25,700 & 25,252 & 0.53 \\
\hline 2016b & 17,416 & 17,283 & 0.49 \\
\hline 2017a & 30,375 & 30,105 & 0.53 \\
\hline 2017b & 64,354 & 63,683 & 0.61 \\
\hline 2018a & 55,390 & 54,906 & 0.60 \\
\hline 2018b & 24,205 & 24,094 & 0.56 \\
\hline $2019 \mathrm{a}$ & 18,277 & 18,211 & 0.51 \\
\hline 2019b & 18,570 & 18,515 & 0.48 \\
\hline 2020a & 15,507 & 15,459 & 0.50 \\
\hline 2020b & 7,949 & 7,935 & \\
\hline
\end{tabular}




\begin{tabular}{|c|c|c|c|}
\hline Topic_Num & Count_of_Comments & Perc_Contrib & Topic_Keywords \\
\hline 0 & 2419 & 0.0804 & 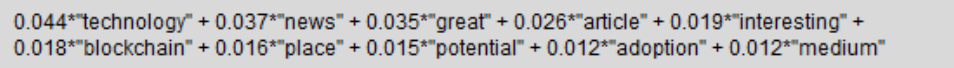 \\
\hline 1 & 1940 & 0.0644 & 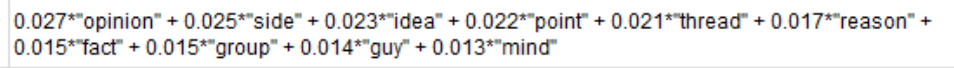 \\
\hline 2 & 1940 & 0.0644 & 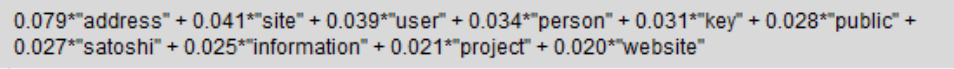 \\
\hline 3 & 2356 & 0.0783 & 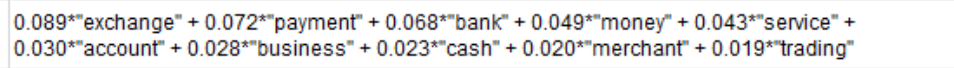 \\
\hline 4 & 952 & 0.0316 & 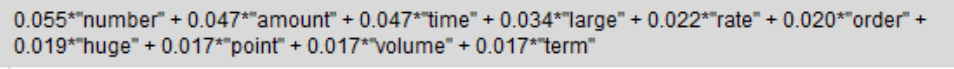 \\
\hline 5 & 3068 & 0.1019 & 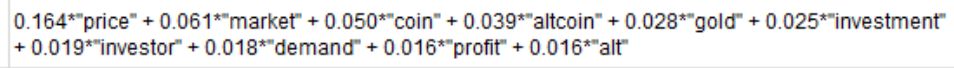 \\
\hline 6 & 1806 & 0.06 & 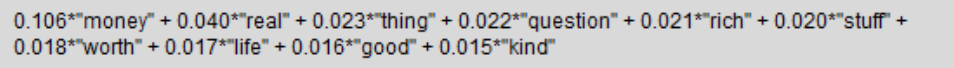 \\
\hline 7 & 2881 & 0.0957 & 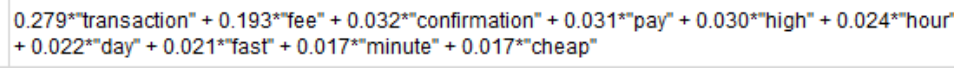 \\
\hline 8 & 1984 & 0.0659 & 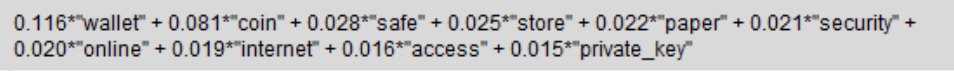 \\
\hline 9 & 1949 & 0.0647 & 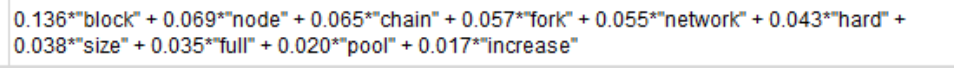 \\
\hline 10 & 1561 & 0.0519 & 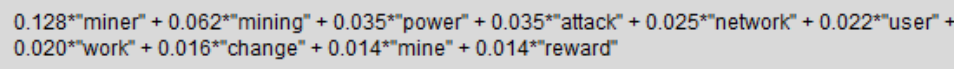 \\
\hline 11 & 1347 & 0.0447 & 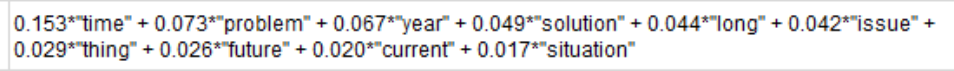 \\
\hline 12 & 2247 & 0.0746 & 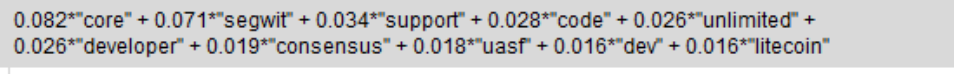 \\
\hline 13 & 1062 & 0.0353 & 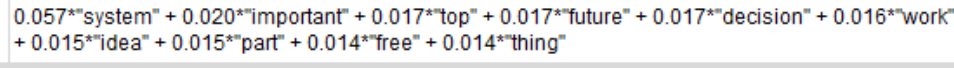 \\
\hline 14 & 2593 & 0.0861 & 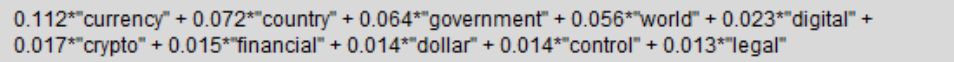 \\
\hline
\end{tabular}

Figure 6: Sample summary table for 2017a dataset. 
- Pyldavis chart This chart shows the 30 most frequent words in each topic and their presence in the rest of corpus.

- Table of all posts In this table we can see all the posts of the corpus and the major topic that could be assigned to each one of them, as well as the probability of this major topic for this specific post.

\subsubsection{Manual topic labeling}

We performed the topic labeling for all the 330 different topics (15 different sets of words in 22 different datasets) we had. The manual process was conducted by two coders who followed these steps:

- Step 1: Reading several documents about the cryptocurrency domain and reviewing them together to gain a similar understanding about the domain.

- Step 2: Reviewing list of the most frequent words of topics in two datasets independently and labeling the topics accordingly.

- Step 3: Discussing their justifications to come to an agreement for choosing a label for the topics in these two datasets.

- Step 4: Repeating Step 2 and Step 3 for two other datasets.

- Step 5: Having discussion meetings and summarizing the list of topics that coders had faced while labeling the first four datasets and building a map for the topics.

- Step 6: Performing the initial manual topic labeling for another six datasets separately to finalize them in the meeting.

- Step 7: Based on the mutual agreement and common point of view in the Step 6 , the coders have decided to continue the process together and labeled the rest 
of datasets and completed the map of topics by adding new topics when they found new ones.

- Step 8: In order to be consist with our labelling, we re-labeled the first four datasets based on the final map of topics.

After the manual topic labeling, we decided to ignore the first dataset(2010a), since there were only about 500 posts in the dataset and we were not able to detect accurate labels for it. We also compared the performance of our topic labeling in different datasets and reviewed the list of frequent words in each topic and revised our topics to make sure that the labels best suited the topics. We checked and finalized the topics for around 40 topic labels that we thought needed a revision.

\subsubsection{Sentiment Analysis}

Sentiment analysis provides high-level categorization of text in terms of polarity (positive vs. negative) or subjectivity (the level of emotion or neutrality of the text). With the high demand for this technique in industry like analyzing customers emails, comments on social media and websites, many ready to use tools are developed and commercialized [78]. Similar to previous work [64], we applied the Valence Aware Dictionary and Sentiment Reasoner (VADER) sentiment analysis on our dataset and calculated the sentiment polarity score for each post. VADER is a lexicon and rulebased tool which is specially designed for social media data [67]. This algorithm assigns each post a compound score from the range of $[-1,1]$, for highly negative to highly positive posts. For our analysis, we also divided all posts into three groups, i.e., negative, neutral, and positive. For classifying the posts based on their sentiment weight we needed a threshold to place them into three categories (negative, positive, neutral). According to the tutorial of this tool on GitHub ${ }^{5}$ the thresholds are defined

\footnotetext{
${ }^{5}$ https://github.com/cjhutto/vaderSentiment
} 
Table 4: Sentiment compound score threshold.

\begin{tabular}{|c|c|}
\hline Sentiment & Scoring \\
\hline Positive & compound score $>0.20$ \\
\hline Neutral & (compound score $>=-0.20)$ and $($ compound score $<=0.20)$ \\
\hline Negative & compound score $<-0.20$ \\
\hline
\end{tabular}

as follows:

- Positive sentiment: compound score $>=0.05$

- Neutral sentiment: compound score $>-0.05$ and compound score $<0.05$

- Negative sentiment: compound score $<=-0.05$

Some researchers $[79,80]$ have already adopted these thresholds. However, considering that using these thresholds for our work, only 8.7 percent of posts were placed in neutral class and this matter made it challenging for us to interpret the results accurately. Therefore, similar to Kim et al. 64, a previous research on bitcointalk.org dataset in 2016, we decided to consider 0.2 as the border between neural and positive posts and -0.2 as the border between negative and neutral posts. Table 4 shows the thresholds we used for classifying posts.

\subsubsection{Data Analysis}

In order to answer our research questions RQ1 to RQ4, we imported all the findings of previous steps 3.2.3, 3.2.4, and 3.2.5 into a PowerBI so that we can build the connection between different output tables and analyze the results. We first assigned the labels (that we had identified in Section 3.2.4 to each topic. Furthermore, we integrated the sentiment information of the posts with the information of all the topics' tables to lay the foundation for our final analysis and evaluation. 
We used Pearson correlation to identify potential relations between topics/sentiment and Bitcoin price. The Pearson correlation coefficient is a statistical measure that quantifies the extent to which two or more variables fluctuate together 81. This metric is sensitive only to a linear relationship between two or more variables. Pearson correlation is usually denoted with $\rho$ or $r$ and it ranges from -1 to +1 . Therefore, we have three interpretations:

(a) a correlation close to zero indicates little or no relationship between the two variables;

(b) a correlation close to one implies that the variables have a tight dependency;

(c) a correlation close to -1 emphasizes that when one variable decreases,other the one also increases and vise versa

The Pearson correlation coefficient is defined as follows. Consider that there are two variables $X$ and $Y$, with $X_{1}, X_{2}, \ldots, X_{n}$ and $Y_{1}, Y_{2}, \ldots, Y_{n}$. Let the mean of $X$ be $\bar{X}$, the standard deviation of $X$ be $S_{x}$, the mean of $Y$ be $\bar{Y}$ and, the standard deviation of $Y$ be $S_{y}$. Pearson's $r$ is:

$$
r=\frac{\sum\left(\frac{x_{i}-\bar{x}}{S_{x}}\right)\left(\frac{y_{i}-\bar{y}}{S_{y}}\right)}{n-1}
$$

In order to calculate the correlation ratio between the input parameters and Bitcoin price, we considered the following inputs to our model: the number of posts, the percentage of posts in its dataset, the average and percentage of positive, neutral, and negative sentiment polarity and, the average Bitcoin price within the time period of the dataset. 


\subsection{Tools and Libraries Used}

All the implementation described in Section 3.2 was done with Python 3.8.3 64bit in a Jupyter Notebook [82] in Visual Studio Code 1.49.0 [83. We used the following open source libraries in our implementation in Python: re [84, nltk [85], and spaCy [86] for data preprocessing; gensim [87] and Mallet LDA 2.0.8 [88] for topic modeling; pyLDAvis [89] for topic model visualisation; numpy [90] for Numpy arrays; pandas [91] for dataframe, and Vader Sentiment 3.3.2 [92] for sentiment analysis.

For generating the results we combined and processed all the data with PowerBI version 2.87.923.0 [93] and built the visuals in its environment or in Tableau [94]. For the correlation model we used R programming language version 3.6.1 [95], ggplot2 [96], ggthemes [97], dplyr [98], corrgram [99], and corrplot [100] libraries. 


\section{Chapter 4}

\section{Analysis and Results}

In this chapter, we present the results for the research questions described in Section 1.3. In each section, the results provide the answer for each research questions of this work.

\subsection{RQ1: What are the discussions in a Bitcoin specialized forum about?}

One of the key results of this work was identifying a variety of distinct topics in the discussions on the bitcointalk org forum. During the process of manual topic labeling as described in Section 3.2.4, we gradually built a map of discussion topics. The definition of these topics is presented in Table 5, and the list of the frequent words for each topic can be found in Table 6 .

While we found 33 distinct topics, we eliminated two unrelated topics and classify 31 topics into six categories containing two or three subcategories:

1. Future: this category refers to the topics related to the predictions about the future of Bitcoin, the way it might change the finance domain, and the role that this cryptocurrency will play in the world in the future. Three topics belong 
Table 5: Map of topics.

\begin{tabular}{|c|c|}
\hline Topic & Description \\
\hline Bitcoin Transaction & How Bitcoin is transferred with Bitcoin wallet in the block chain network. \\
\hline Bitcoin Alternatives & $\begin{array}{l}\text { Discussion related to the other alternative cryto currencies to Bitcoin like } \\
\text { Altcoins or Etherium. }\end{array}$ \\
\hline Bitcoin Exchange Rate & $\begin{array}{l}\text { What is the value of bitcoin for the purpose of exchange with other assets like } \\
\text { gold. }\end{array}$ \\
\hline Bitcoin Foundation & The Bitcoin Foundation is an American nonprofit corporation founded in 2012. \\
\hline Bitcoin Future Price Discussion & Discussion related to the prediction of Bitcoin price in future. \\
\hline Bitcoin in Illegal Activity & How can Bitcoin used by the criminals in the illegal activity. \\
\hline Bitcoin Price Discussion & Discussion related to Bitcoin Price in short term. \\
\hline Bitcoin Price Discussion (Covid) & How Bitcoin price has been effected during the Pandamic. \\
\hline Bitcoin Regulatory & $\begin{array}{l}\text { The law and the rules imposed by the government on the exchange and trade } \\
\text { on Bitcoin. }\end{array}$ \\
\hline Bitcoin Scam & Discussion related to the exposing the fraud that puts Bitcoin users to theft. \\
\hline Bitcoin Trading & Discussion related to the risks in trading Bitcoin. \\
\hline Bitcoin Transaction Cost/Time & The cost/time of sending the bitcoin over the network. \\
\hline Bitcoin User experience & Discussion of the experiences of each person with using and trading the Bitcoin. \\
\hline Blockchain Technology & Discussion related how the block chain technology and its applications. \\
\hline Cryptocurrency Adoption & Acceptance and use of cryto currencies by the public. \\
\hline Discussion of an Event & Discussion of special/unknown events in forum. \\
\hline Effect of Bitcoin on Banking & $\begin{array}{l}\text { Impact of Bitcoin on banking system, How financial instituate act related to } \\
\text { Bitcoin. }\end{array}$ \\
\hline Effects of Covid on Bitcoin & What are the effects of COVID in the Bitcoin economy. \\
\hline Financial Return & $\begin{array}{l}\text { Discussion related to how much money made or lost on Bitcoin investment } \\
\text { over some period of time. }\end{array}$ \\
\hline Fork & $\begin{array}{l}\text { Discussion related to changes to the blockchain protocol or when a blockchain } \\
\text { diverges into two potential paths forward. }\end{array}$ \\
\hline Future of Bitcoin & what happens to the value of Bitcoin in future? \\
\hline Future Role of Bitcoin & The role of Bitcoin in the world, its role in the economy and other fields. \\
\hline General Bitcoin Discussion & Discussion about the origin of Bitcoin/ Satoshi Nakamoto. \\
\hline General Network Discussion & Discussion related to the general queries or issues related to network. \\
\hline Mining: Requirement & $\begin{array}{l}\text { What are the hardware and other requierments requiered for mining. And the } \\
\text { financial return of mining. }\end{array}$ \\
\hline Mining: transactions & $\begin{array}{l}\text { How mining secures the network of block chain and confirms the Bitcoin trans- } \\
\text { action. The } 51 \% \text { attack in the network. }\end{array}$ \\
\hline Network Security & Discussion related to security of network. \\
\hline Off Topic & Discussion related to the topics that are not relevant to \&\& Bitcoin. \\
\hline Payment with Bitcoin & $\begin{array}{l}\text { How to use Bitcoin for payment and ultimately move that amount to bank } \\
\text { account. }\end{array}$ \\
\hline Project Development & Organization of Bitcoin and related projects, campaigns, advertising etc. \\
\hline Technical Issue & Discussion related to the technical issue. \\
\hline User Security & This topic is about the safety of users wallet, it's security and the way it works. \\
\hline Wallet Apps & Discussion related to the Software that stores Bitcoin. \\
\hline
\end{tabular}


Table 6: Frequent words in each topic.

\begin{tabular}{|c|c|}
\hline Topic & Frequent words \\
\hline Bitcoin Alternatives & coin problem price number gold market internet datum \\
\hline Bitcoin Exchange Rate & money currency price system payment bank dollar \\
\hline Bitcoin Foundation & work project price source market support idea \\
\hline Bitcoin Future Price Discussion & price time year long problem number investment profit \\
\hline Bitcoin in Illegal Activity & user illegal criminal crime drug information issue scam \\
\hline Bitcoin Price Discussion & price coin currency market increase profit worth crypto \\
\hline Bitcoin Price Discussion (Covid) & price market time pandemic increase rate stock high \\
\hline Bitcoin Regulatory & government law country regulation state world legal currency \\
\hline Bitcoin Scam & scam project website site hack money worth account \\
\hline Bitcoin Trading & exchange cryptocurrency trading user trade amount Investment profit \\
\hline Bitcoin Transaction & exchange address payment system site account cryptocurrency money \\
\hline Bitcoin Transaction Cost/Time & transaction fee payment amount user block service network \\
\hline Bitcoin User Experience & service person user good trust idea reason part product \\
\hline Blockchain Technology & technology project blockchain internet indusatry development datum \\
\hline Cryptocurrency Adoption & adoption future crypto cryptocurrency gold altcoin time news \\
\hline Discussion of an Event & idea site thread business free question video thread \\
\hline Effect of Bitcoin on Banking & money currency bank system world financial payment service \\
\hline Effects of Covid on Bitcoin & news crypto trading situation due altcoin virus pendamic \\
\hline Financial Return & worth price exchange coin currency market time risk \\
\hline Fork & block node chain fork core network segwit change \\
\hline Future of Bitcoin & coin idea time number risk person trust bit \\
\hline Future Role of Bitcoin & currency crypto money world future time idea \\
\hline General Bitcoin Discussion & system currency idea coin real work satoshi public \\
\hline General Network Discussion & internet user client source blockchain software change information \\
\hline Mining: Transactions & transaction fee mining miner block network cost attack \\
\hline Mining: Requirement & miner block mining network cost power coin world \\
\hline Network Security & network time client number node point amount attack \\
\hline off topic & idea thing thread question video great question world \\
\hline Payment with Bitcoin & currency service account fee payment service exchange money \\
\hline Project Development & news site article project website page \\
\hline Technical Issue & system problem bit time person work issue secuity \\
\hline User Security & wallet address key public security safe paper information \\
\hline Wallet Apps & address account password email site website exchange service \\
\hline
\end{tabular}


to this category such as "Cryptocurrency Adoption", "Future of Bitcoin" and "Future Role of Bitcoin".

2. General: Some of the general discussions such as introducing blockchain technology, defining Bitcoin or introducing payment and transaction methods are placed to this category. Moreover, this category concerns the topics that discuss the measures that should be taken to increase public awareness about Bitcoin and establishing specific institutions. Some of the topics that could be found in this category are "Bitcoin Transaction Cost/Time", "Blockchain Technology" and "Effects of COVID on Bitcoin".

3. Mining: This category contains two different topics, one of them is the requirement for mining and the feasibility studies. The other one is related to its role and description during transactions, as well as discussions about the possibility of $51 \%$ attacks from miner sides.

4. Monetary: A large number of topics are related to this category and we further classify them into three subcategories which are Currency, Financial, and Price. In the Currency subcategory, there are some discussions related to the currency feature of Bitcoin (two topics) and one for other cryptocurrencies posts. Financial subcategory contains three topics related to the trading and investment of Bitcoin, its risk and financial returns. Finally, the Price subcategory is for all topics related to Bitcoin price.

5. Regulations: This category contains all the discussions related to the governments, adopting Bitcoin in illegal activities or scamming. The posts in this category of topics are usually related to making new regulations for Bitcoin, concerns and ideas about Bitcoin tax, the situation in different countries and some political events related to Bitcoin. 
6. Technical: This category contains six different topics, and we classified them in two subcategories, Wallet and Others. Wallet subcategory has two topics, the first one is related to the applications that can be used for managing personal accounts. The other topic is related to the wallet and users' security. The rest of topics are placed in the Others subcategory. Here, one topic is related to the technical problems that occurred and the discussions about some technical issues. Two other topics are related to general and security aspects of the network. Finally, the last topic is about the fork conditions in mining and the related discussions.

Figure 7 represents distribution of topics among the above mentioned six categories. We processed 543,363 posts, and as we can see, Monetary with around 30 percent of posts is the largest category. The number of posts in this category $(162,335)$ virtually doubles the number of posts in the next major categories which are General and Technical and posts of these two categories contain around 17 percent and 15 percent of the corpus respectively. Furthermore, Future with around only 5 percent of posts is the smallest category and the number of posts in this category is almost half of the posts of the next small categories which are Regulations and Mining with 9 percent and 10 percent of posts. As Figure 7 presents, there is a un-labeled category with around 15 percent of posts which contains comments that we could not assign to any specific topic and for simplicity we considered them as Off-topic.

Table 7 depicts the categorization of topics along with the number of their occurrences and detailed information about the number of posts for each topic. As we can observe, some of the categories such as Monetary and Technical have subcategories, while categories such as General and Future do not have any subcategory. We assign each one of the 31 topics to one of these categories. According to this table, we can see that some topics such as "User Security" and "Bitcoin Regulatory" and 


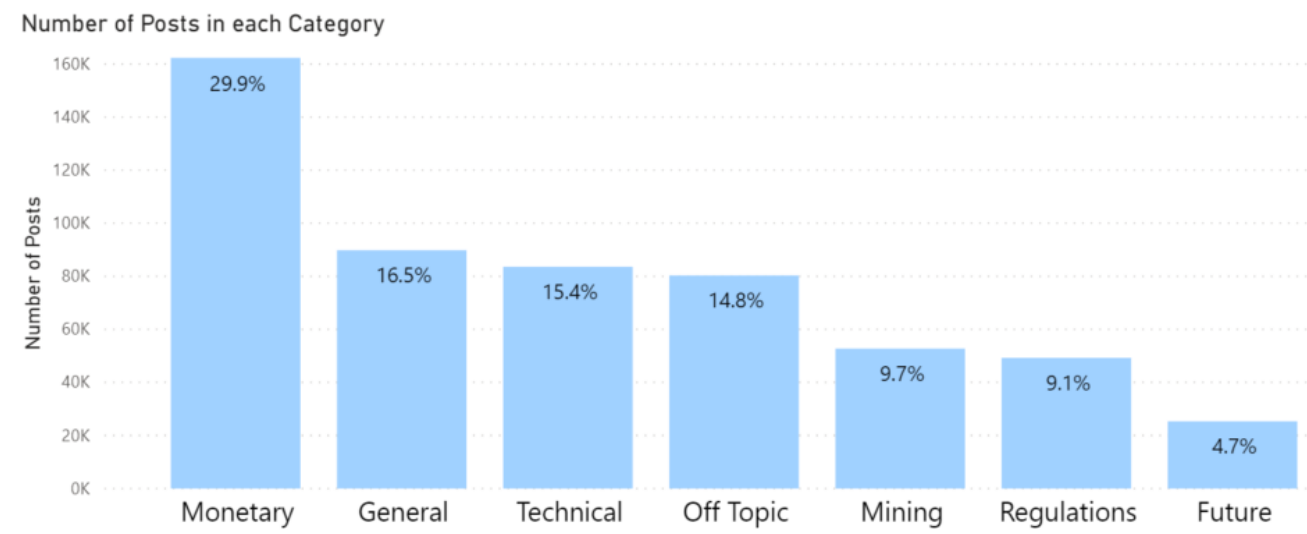

Figure 7: Number of posts in categories.

"Bitcoin Exchange Rate" have been quite repetitive in discussions and users of the forum had been continuously talking about them. Some other topics have shown up in more than half of datasets such as "Future Role of Bitcoin" and "Mining: Requirement". Furthermore, we can see that a number of topics have appeared only over some periods and have a frequency of 6 to 11 times such as "Effect of Bitcoin on Banking", "Blockchain Technology", and "Bitcoin Alternatives". Finally, as Table 7 shows, some discussion topics have been quite uncommon and repeated in only fewer than 5 sets. Our justification is that these topics are related to a specific event or phenomenon that attracted public opinion only for a short period of time such as "Bitcoin Price Discussion (COVID)". 


\section{Answer to RQ1:}

In this work, we identified 31 distinct topics in www.bitcoing.org that people talk about and categorized these 31 topics to 6 categories. While some topic categories such as Monetary are more common having a considerable percentage of posts (around $30 \%$ ), several topics are repetitive which means they persist in different time periods and people always discuss these matters, such as Monetary or Technical. On the other hand, some other topics are not major, consistent and unlikely to appear in many sets and over a long period of time.

\subsection{RQ2: How do the discussion topics on the fo- rum evolve over the time?}

In the second research question, we study and analyze the evolution of discussion topics over the time. In order to reach this goal, we first present the overall change trend of the six categories which we introduced in the previous section. And then, we investigate the evolution patterns of each category in detail.

Figure 8 illustrates the number of existing discussion topics in each category for each dataset. As we can observe, the most frequent category of the topics is Monetary as it forms more than $33 \%$ of the debates. General category, on the other hand, appears with various frequencies within different time spans. However, it usually appears in at least one of the discussion topics. Mining category which has two topics usually can be found in discussions. Future and Regulations appear in discussions occasionally and sometimes they show up in debates and sometimes they disappear.

In order to explore the evolution of discussion topics more closely, we study the change of discussion topics in each category. 
Table 7: Number of posts and frequency of topics in each category.

\begin{tabular}{|c|c|c|c|c|}
\hline Category & Subcategory & Selected label & Frequency & \# Posts \\
\hline \multirow{3}{*}{ Future } & \multirow{3}{*}{-} & Cryptocurrency Adoption & 3 & 2,299 \\
\hline & & Future of Bitcoin & 5 & 4,869 \\
\hline & & Future Role of Bitcoin & 13 & 18,157 \\
\hline \multirow{8}{*}{ General } & \multirow{8}{*}{-} & Bitcoin Transaction & 11 & 16,246 \\
\hline & & Bitcoin Foundation & 3 & 5,224 \\
\hline & & Bitcoin Transaction Cost/Time & 7 & 9,183 \\
\hline & & Bitcoin User Experience & 1 & 2,030 \\
\hline & & Blockchain Technology & 8 & 13,521 \\
\hline & & Effects of Covid on Bitcoin & 1 & 847 \\
\hline & & General Bitcoin Discussion & 11 & 17,368 \\
\hline & & Project Development & 13 & 25,411 \\
\hline \multirow{2}{*}{ Mining } & \multirow{2}{*}{-} & Mining : Transactions & 17 & 36,008 \\
\hline & & Mining: Requirement & 12 & 16,730 \\
\hline \multirow{9}{*}{ Monetary } & \multirow{3}{*}{ Currency } & Bitcoin Alternatives & 8 & 15,323 \\
\hline & & Bitcoin Exchange Rate & 16 & 28,166 \\
\hline & & Payment with Bitcoin & 14 & 30,681 \\
\hline & \multirow{3}{*}{ Financial } & Bitcoin Trading & 3 & 3,177 \\
\hline & & Effect of Bitcoin on Banking & 8 & 13,989 \\
\hline & & Financial Return & 10 & 23,517 \\
\hline & \multirow{3}{*}{ Price } & Bitcoin Future Price Discussion & 9 & 13,249 \\
\hline & & Bitcoin Price Discussion & 10 & 33,497 \\
\hline & & Bitcoin Price Discussion (COVID) & 1 & 736 \\
\hline \multirow{3}{*}{ Regulations } & \multirow{3}{*}{-} & Bitcoin in Illegal Activity & 4 & 8,836 \\
\hline & & Bitcoin Regulatory & 18 & 37,415 \\
\hline & & Bitcoin Scam & 3 & 2,986 \\
\hline \multirow{6}{*}{ Technical } & \multirow{4}{*}{ Others } & Fork & 7 & 17,637 \\
\hline & & General Network Discussion & 2 & 2,689 \\
\hline & & Network Security & 2 & 812 \\
\hline & & Technical Issue & 7 & 7,080 \\
\hline & \multirow{2}{*}{ Wallet } & User Security & 20 & 42,759 \\
\hline & & Wallet Apps & 7 & 12,601 \\
\hline
\end{tabular}




\begin{tabular}{|c|c|c|c|c|c|c|c|c|c|c|c|c|c|c|c|c|c|c|c|c|c|c|}
\hline Category & $\begin{array}{l}\text { No } \\
\text { 。्̊ }\end{array}$ & 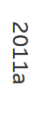 & 莽 & 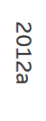 & $\begin{array}{l}\text { N } \\
\stackrel{0}{N} \\
\text { V. }\end{array}$ & 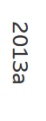 & 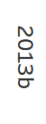 & 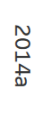 & 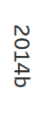 & \begin{tabular}{l}
$N$ \\
$\stackrel{O}{0}$ \\
\multirow{N}{N}{}
\end{tabular} & $\begin{array}{l}\text { N } \\
\stackrel{0}{U} \\
\sigma\end{array}$ & $\begin{array}{l}\text { OO } \\
\stackrel{0}{0}\end{array}$ & $\begin{array}{l}\stackrel{N}{\circ} \\
\stackrel{\circ}{\sigma}\end{array}$ & $\underset{\sim}{\stackrel{O}{0}}$ & \begin{tabular}{l}
$\stackrel{N}{0}$ \\
\multirow{\sigma}{\sigma}{}
\end{tabular} & 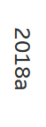 & $\begin{array}{l}\text { N } \\
\stackrel{0}{0} \\
\text { O) }\end{array}$ & $\begin{array}{l}\text { N } \\
\stackrel{0}{0} \\
\stackrel{2}{2}\end{array}$ & $\begin{array}{l}\tilde{N} \\
\stackrel{0}{\omega} \\
\sigma\end{array}$ & $\begin{array}{l}\text { O } \\
\text { O } \\
\text { D }\end{array}$ & $\begin{array}{l}\tilde{N} \\
\text { O } \\
\text { V }\end{array}$ & 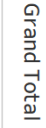 \\
\hline Future & & 2 & 1 & 1 & 2 & & 1 & 1 & 1 & & 1 & & 1 & 2 & & 1 & 1 & 1 & 1 & 1 & 3 & 21 \\
\hline General & 2 & 2 & 1 & 1 & 2 & 3 & 3 & 2 & 3 & 2 & 3 & 3 & 3 & 3 & 2 & 3 & 2 & 4 & 4 & 4 & 3 & 55 \\
\hline Mining & 2 & 1 & 1 & 2 & 2 & 2 & 1 & 1 & 1 & 1 & 2 & 2 & 2 & 1 & 1 & 1 & 1 & 1 & 1 & 2 & 1 & 29 \\
\hline Monetary & 3 & 4 & 3 & 2 & 2 & 3 & 3 & 4 & 4 & 5 & 3 & 4 & 3 & 3 & 5 & 7 & 5 & 5 & 4 & 5 & 2 & 79 \\
\hline Regulations & 1 & 1 & 2 & 1 & 1 & 1 & 1 & 2 & 1 & 2 & & & & 1 & 1 & 1 & 1 & 1 & 2 & 2 & 3 & 25 \\
\hline Technical & 3 & 2 & 2 & 3 & 2 & 1 & 3 & 1 & 2 & 3 & 3 & 3 & 2 & 2 & 3 & 1 & 3 & 2 & 1 & 1 & 2 & 45 \\
\hline Grand Total & 11 & 12 & 10 & 10 & 11 & 10 & 12 & 11 & 12 & 13 & 12 & 12 & 11 & 12 & 12 & 14 & 13 & 14 & 13 & 15 & 14 & 254 \\
\hline
\end{tabular}

Figure 8: Presence of the topics in each categories for each dataset.

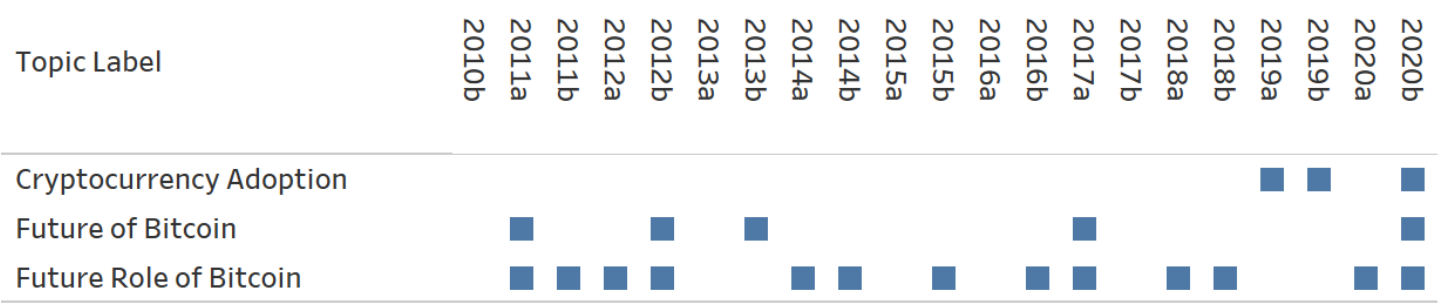

Figure 9: Topics over the time in Future category.

\subsubsection{Future}

In the Future category, we can find three topics and the most repetitive one is "Future Role of Bitcoin". This topic has been repeated in 13 datasets and could be taken as an ongoing and continuing topic. The next topic is "Future of Bitcoin" which has been repeated in 5 datasets and mostly has appeared in the first years after Bitcoin's birth during which people discussed the future of Bitcoin. This topic has been replaced by a third topic called "Bitcoin Adoption" in the last 2 years, and we can see that the topics of debates in the forum have changed and instead of discussing the existence or extinction of Bitcoin, people talk about its stability and adoption. The trend of these changes has been depicted in Figure 9.

\subsubsection{General}

During the earliest years, "Project Development" was the most frequent topic of this category. Although we still can see this topic in the General category, its frequency 


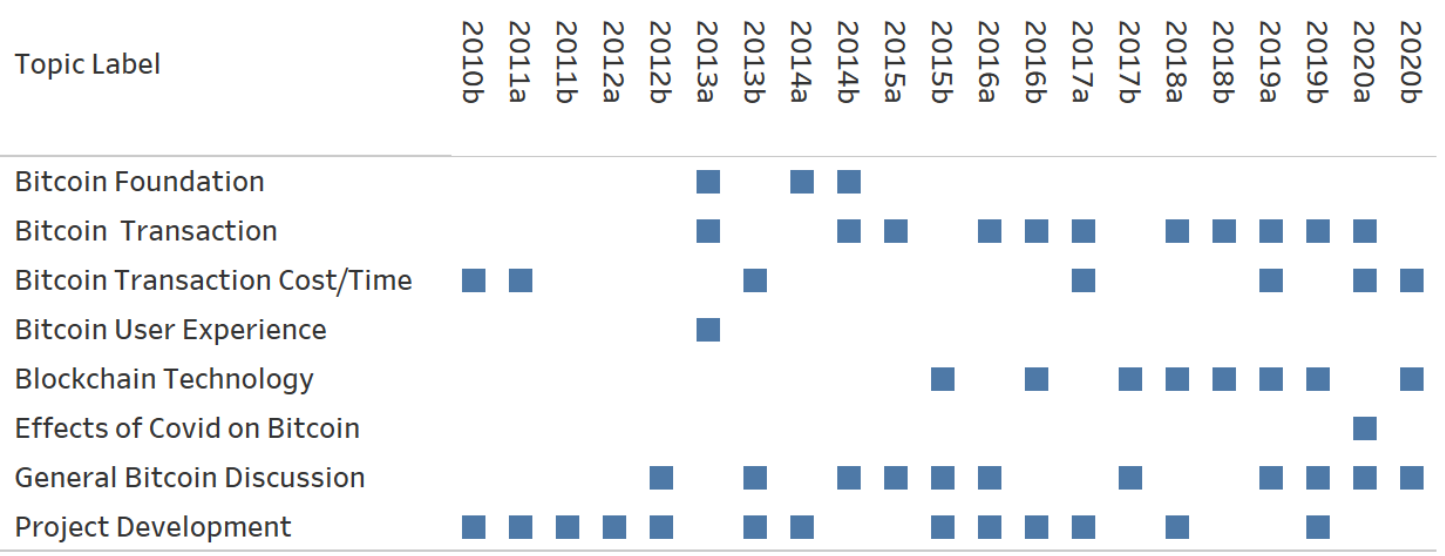

Figure 10: Topics over the time in General category.

has decreased over the time. From 2012, two other topics such as "Bitcoin Transaction" and "General Bitcoin Discussion" have been created and they still remain popular discussion topics. "Bitcoin Transaction Cost/Time" is a topic that has appeared occasionally in some datasets, and we still can find some traces of this topic in the forum discussions occasionally. In 2015 a topic that we labeled as "Blockchain Technology" showed up, and it is still an alive and ongoing topic. As we can see in Figure 10, there is a topic called "Bitcoin Foundation" among the topics of this category. This topic was created following the establishment of a NGO with the same name in the USA in 2013 and 2014. Finally, "Bitcoin User Experience" and "Effects of COVID on Bitcoin" are two topics that have been appeared only once among all the 22 datasets.

\subsubsection{Mining}

There are two topics in this category, and each one of these topics has been repeated in more than half of the time periods. The topics which are related to transactions and risk attacks have been discussed continuously, but the one which is related to feasibility and requirements has appeared or disappears periodically. The repeating 


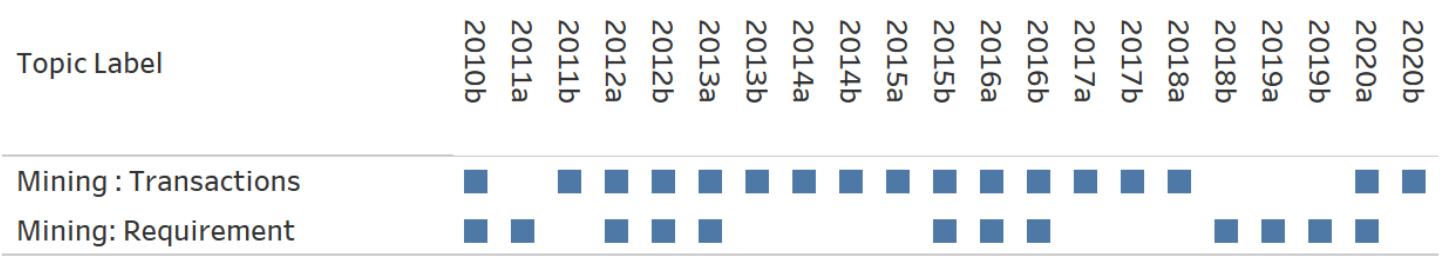

Figure 11: Topics over the time in Mining category.

\begin{tabular}{|c|c|c|c|c|c|c|c|c|c|c|c|c|c|c|c|c|c|c|c|c|c|c|}
\hline ategory & $\begin{array}{l}\text { N } \\
\text { 品 } \\
\text { 인 }\end{array}$ & $\begin{array}{l}\stackrel{\sim}{\circ} \\
\stackrel{\rho}{\ominus}\end{array}$ & 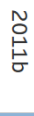 & 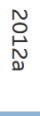 & $\begin{array}{l}\underset{N}{\stackrel{0}{0}} \\
\underset{\sim}{\sim}\end{array}$ & 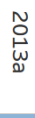 & $\underset{\underset{\sigma}{\tilde{\omega}}}{\stackrel{\widetilde{\rho}}{\omega}}$ & $\underset{\text { D }}{\stackrel{N}{P}}$ & 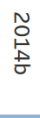 & $\begin{array}{l}\tilde{O}_{0} \\
\text { Un }\end{array}$ & $\begin{array}{l}\text { OO } \\
\text { Un } \\
\sigma\end{array}$ & $\begin{array}{l}\text { Oे } \\
\text { O⿱口口) }\end{array}$ & $\begin{array}{l}\text { O⿱ } \\
\text { g } \\
\sigma\end{array}$ & $\underset{\mathcal{N}}{\tilde{S}}$ & $\underset{0}{\stackrel{0}{ٍ ~}}$ & 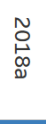 & $\begin{array}{l}\underset{0}{0} \\
\stackrel{\infty}{\infty}\end{array}$ & 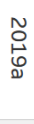 & $\begin{array}{l}\tilde{0} \\
\stackrel{0}{0} \\
\sigma\end{array}$ & $\begin{array}{l}\tilde{N} \\
\text { Õ } \\
\text { D. }\end{array}$ & $\begin{array}{l}\tilde{\tilde{N}} \\
\text { Oे }\end{array}$ & $\begin{array}{l}\frac{1}{20} \\
\frac{2}{2} \\
\frac{1}{0} \\
0\end{array}$ \\
\hline Currency & 3 & 2 & 2 & 2 & 2 & 2 & 2 & 3 & 2 & 1 & 1 & 2 & 2 & 2 & 2 & 3 & 1 & 1 & 1 & 2 & & 38 \\
\hline Financial & & 1 & & & & & 1 & 1 & 2 & 2 & 2 & 2 & 1 & & 1 & 2 & 2 & 2 & 1 & 1 & & 21 \\
\hline Price & & 1 & 1 & & & 1 & & & & 2 & & & & 1 & 2 & 2 & 2 & 2 & 2 & 2 & 2 & 20 \\
\hline Grand Total & 3 & 4 & 3 & 2 & 2 & 3 & 3 & 4 & 4 & 5 & 3 & 4 & 3 & 3 & 5 & 7 & 5 & s & 4 & 5 & 2 & 79 \\
\hline
\end{tabular}

Figure 12: Topics in Monetary category.

pattern of these two topics are also presented in Figure 11.

\subsubsection{Monetary}

The most frequent topic among all the discussions is related to the Monetary category. This category is divided into three subcategories which are 1) Currency 2) Financial, and 3) Price. In this category, we can find an increase in the diversity of topics and the number of topics in each set grows over the time. For instance, while in the first years there were only three or four topics in this category, this value increased to 7 topics in 2018a. Among the three mentioned subcategories the major topic in the discussions used to be in Currency subcategory. However, from 2013b, the topic of debates changed and gradually users started discussing about topics in the Financial subcategory. This trend still exists in discussions of the forum. The Price subcategory started from 2017a and turned out to one of the major discussion topics in the forum; we need to mention that before 2017 this topic appeared occasionally in around 30 percent of discussions. The trend of all these changes are presented in Figure 12 . 


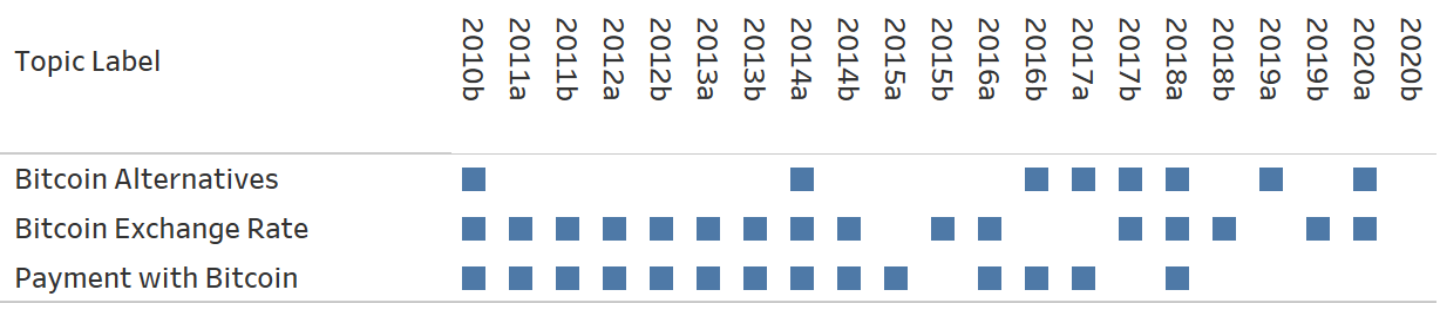

Figure 13: Topics over the time in Currency subcategory.

- Currency In the Currency subcategory, there are three topics which are: "Bitcoin Exchange Rate", "Payment with Bitcoin", and "Bitcoin Alternatives". The first item of the mentioned list (Bitcoin Exchange Rate) appeared in the discussion constantly. "Payment with Bitcoin" on the other hand, disappeared in some time spans. For instance, while it had been a constant member of discussion topics in 2017, in 2018 there is no trace of this topic in the discussions of the forum after 2018 (the last time that this topic appeared was in 2018a). Discussions about "Bitcoin Alternatives" take place time by time and the only time period in which this topic showed up constantly was between 2016 and 2018. The trend of changes is presented in Figure 13.

\section{- Financial}

Financial subsection consists of three topics and the major one is "Financial Return" that occurred occasionally in 2011a and continued to appear continuously between 2013 to 2018 (except the one year period between 2016b and 2017a). Similarly, from 2014 to 2019 "Effect of Bitcoin on Banking" has shown up in discussion constantly. From 2019 a new topic which we labeled as "Bitcoin Trading" which in turn indicated the transition of topics from "Financial Return" to this topic. The repetition trend of topics can be found in Figure 14.

\section{- Price}

Discussion about Price occurred occasionally before 2017, however, from 2017 


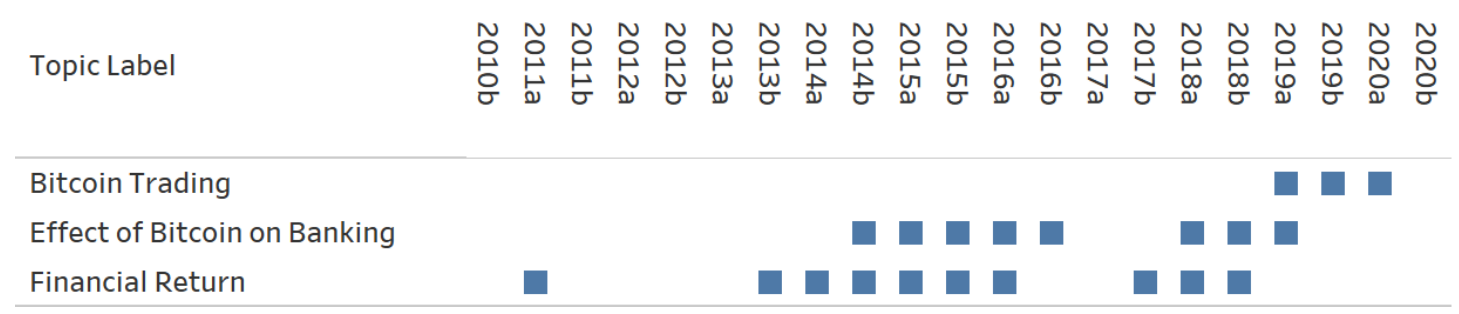

Figure 14: Topics over the time in Financial subcategory.

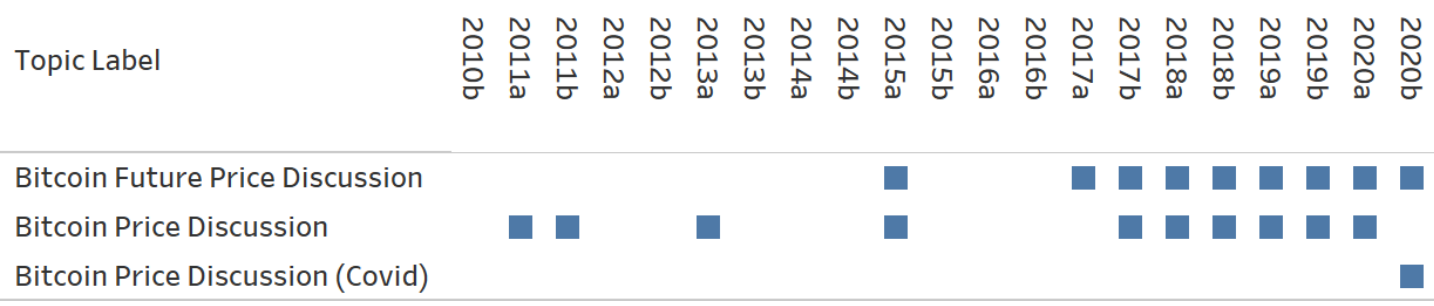

Figure 15: Topics over the time in Price subcategory.

"Bitcoin Price Discussion" has appeared in debates constantly. Furthermore, future prices and price prediction has been added to the discussion topics from 2017 simultaneously. At the end of this period we can find some discussions about the "Bitcoin Price Discussion (COVID)". Figure 15 depicts the repetition trend of these topics.

\subsubsection{Regulations}

The major topic of this category is "Bitcoin Regulatory" which could be considered as one of the continuing discussions in the forum. Except for a 1.5 year long period between 2015 and 2016, we can find this topic in almost all the time periods. "Bitcoin Scam" debates began in 2019. Moreover, discussions regarding illegal activities using Bitcoin have been found occasionally in four datasets. The trend of changes is presented in Figure 16. 


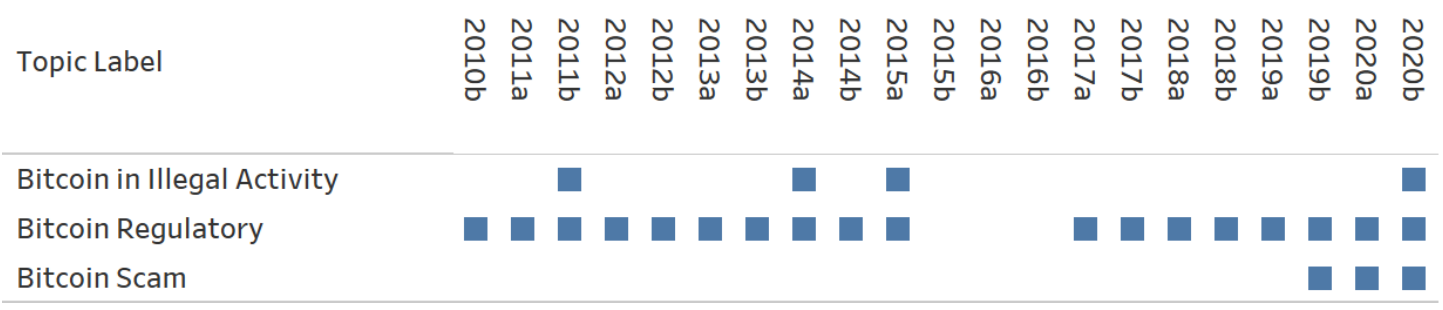

Figure 16: Topics over the time in Regulations category.

\begin{tabular}{|c|c|c|c|c|c|c|c|c|c|c|c|c|c|c|c|c|c|c|c|c|c|c|}
\hline $\begin{array}{l}\text { Sub } \\
\text { Category }\end{array}$ & $\begin{array}{l}\text { O् } \\
\stackrel{0}{0} \\
\text { o }\end{array}$ & 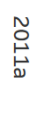 & 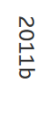 & 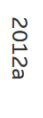 & 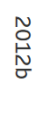 & 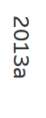 & $\begin{array}{l}\underset{\stackrel{\omega}{\omega}}{\omega} \\
\underset{\sigma}{0}\end{array}$ & 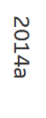 & $\begin{array}{l}\text { OO } \\
\stackrel{0}{0}\end{array}$ & $\begin{array}{l}\text { O } \\
\text { 岕 } \\
\text { N }\end{array}$ & $\begin{array}{l}\text { 号 } \\
\text { G } \\
\sigma\end{array}$ & $\begin{array}{l}\text { О } \\
\text { వ్ర }\end{array}$ & $\begin{array}{l}\stackrel{N}{\circ} \\
\stackrel{\leftrightarrow}{\sigma}\end{array}$ & 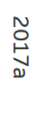 & $\begin{array}{l}\stackrel{0}{\ominus} \\
\forall\end{array}$ & 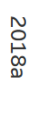 & 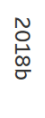 & \begin{tabular}{l} 
O \\
$\stackrel{\leftrightarrow}{0}$ \\
\multirow{2}{*}{}
\end{tabular} & $\begin{array}{l}\tilde{O} \\
\text { Oे } \\
\text { o }\end{array}$ & $\begin{array}{l}\text { No } \\
\text { O్ర }\end{array}$ & $\begin{array}{l}\text { O } \\
\text { Oे }\end{array}$ & 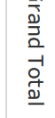 \\
\hline Others & 2 & & & 1 & 1 & & 1 & & 1 & 2 & 2 & 2 & 1 & 1 & 1 & & 2 & & & & 1 & 18 \\
\hline Wallet & 1 & 2 & 2 & 2 & 1 & 1 & 2 & 1 & 1 & 1 & 1 & 1 & 1 & 1 & 2 & 1 & 1 & 2 & 1 & 1 & 1 & 27 \\
\hline Grand Total & 3 & 2 & 2 & 3 & 2 & 1 & 3 & 1 & 2 & 3 & 3 & 3 & 2 & 2 & 3 & 1 & 3 & 2 & 1 & 1 & 2 & 45 \\
\hline
\end{tabular}

Figure 17: Topics in Technical category.

\subsubsection{Technical}

This category has two subcategories which are Wallet and Others. Wallet has shown up in all the time spans, while Others can be found in most of the time periods. The change pattern of these subcategories is presented in Figure 17. Next, we elaborate on each one of them.

- Wallet We assign two topics in this subcategory which are "User Security" and "Wallet Apps". The first one has been repeated all the time. The second one used to appear more frequently at first and nowadays, despite being an ongoing topic, it is less likely to show up among discussions. The repetition trend of topics can be found in Figure 18.

- Others The most frequent topic in this subcategory is "Fork" which appeared constantly between 2015 and 2018, and after this time span, we can find no trace of this topic in discussions. "Technical Issue" is the next topic that has appeared in debates occasionally. The other two topics of this category which are "General Network Discussion" and "Network Security" have occurred only 


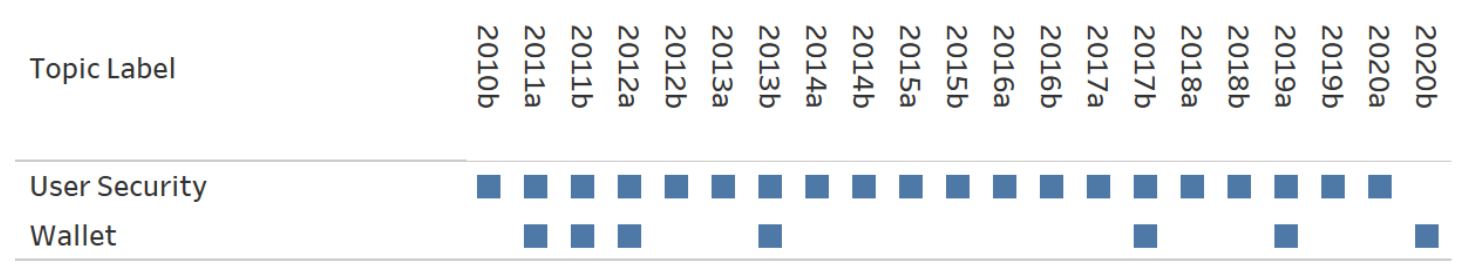

Figure 18: Topics over the time in Wallet subcategory.

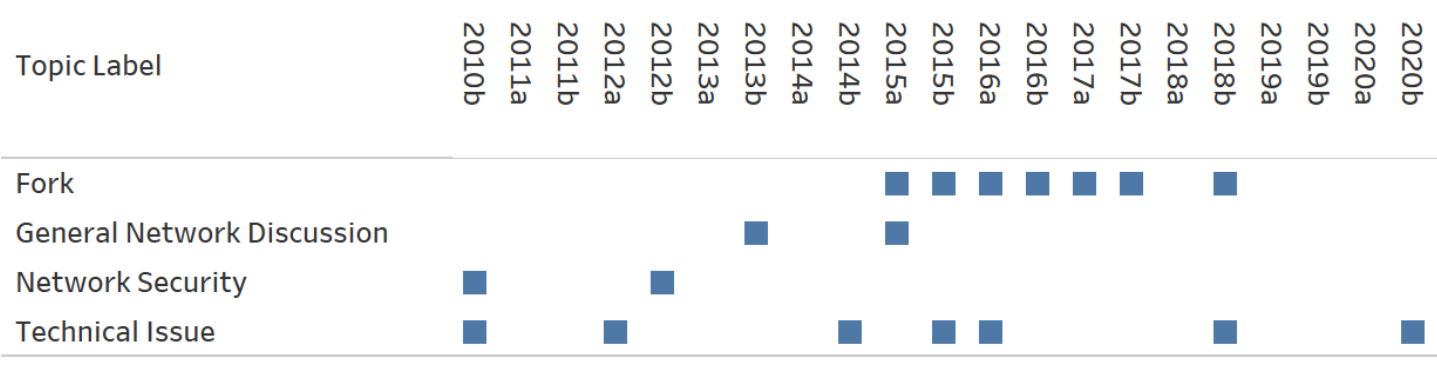

Figure 19: Topics over the time in Others subcategory.

twice in the forum and could be taken as the rare discussion topics. The change trend of topic in presented in Figure 19.

Answer to RQ2: During our analysis of the topics, we found that except for some
topics such as "Mining: Transactions" or "Bitcoin Regulatory" that showed up in the
forum continuously, other topics in the forum have experienced some change over the
time which included some alteration in the discussion topics of a specific category and
creation of temporary topics over some specific periods or an increase in the number
of topics in some categories. Some of the most important changes in discussion topics
are an increase in the diversity of topics in Monetary category, and creation of more
specialized topics such as "Future Price" or "Bitcoin Adoption" in the forum.




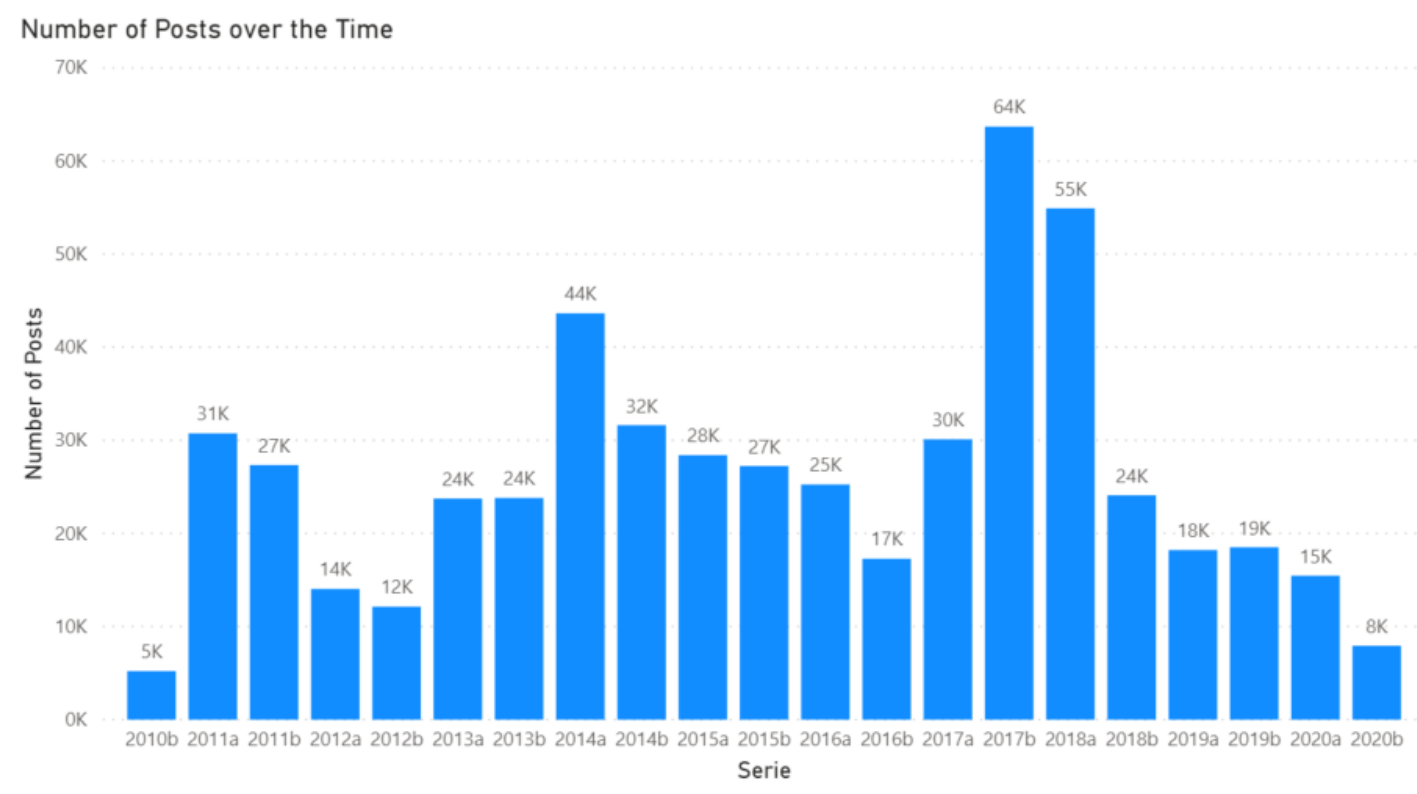

Figure 20: Total number of posts over the time.

\subsection{RQ3: How do the number of posts in each topic change over the time?}

The number of posts in this forum has changed over the time which means the popularity of the forum has varied across the studied timeline. As we can see in Figure 20, the largest number of posts has been written within a one-year long time period (from $2017 \mathrm{~b}$ to $2018 \mathrm{a}$ ), and the second period in which we can see the largest number of comments is 2014a. As this figure suggests, the number of posts has decreased in this forum over the past two years, which is most probably the consequence of the emergence of some papers and research work that used reddit.com as a forum for evaluating comments 8,101 .

Generally the number of posts in a category changes proportionally to the overall increase or decrease in the number of posts. However, the largest value of increase is related to Monetary category and the trend of change in this category is similar to the trend for all the posts. The second large category is General that has virtually 
the same change trend in the number of posts, except for a period between 2017 and 2018 that despite a general increase in the number of posts in the whole sets, number of posts was constant for this category.

Technical category used to have a larger number of posts during the first years, it reduced in size within the recent years. Considering the fact that we can find a similar trend of change in other categories and in the overall average, we will discuss each one in their own part. We will describe the details using two charts: Figure 21 represents the number of post (numerical values) and Figure 22 represents the percentage of each category in its own set, i.e., in $2012 b$.

\subsubsection{Future}

Each one of the topics of this category has appeared in around 5 percent of posts and given the fact that usually only one of them showed up in a dataset, overall, they formed at most 10 percent of the posts. The only exception is $2020 \mathrm{~b}$ in which the three topics occurred together and formed more than 15 percent of the whole posts. The trend of changes in this category has been depicted in Figure 23 and Figure 24.

\subsubsection{General}

"Project Development" was one of the important topics in the first years with around 10 percent of all posts of the forum in that time. This topic still could be found in discussions occasionally. The second important topic in this category is "Blockchain Technology" which could be assigned to around 5 percent of the discussions of the forum. The posts that are related to this topic have been written since 5 years ago. The next topic of this category is "Bitcoin Transaction" which again appears in around 5 percent of debates of the forum. The information about the number and the percentage of each one of the topics of this category is depicted in Figure 25 and 


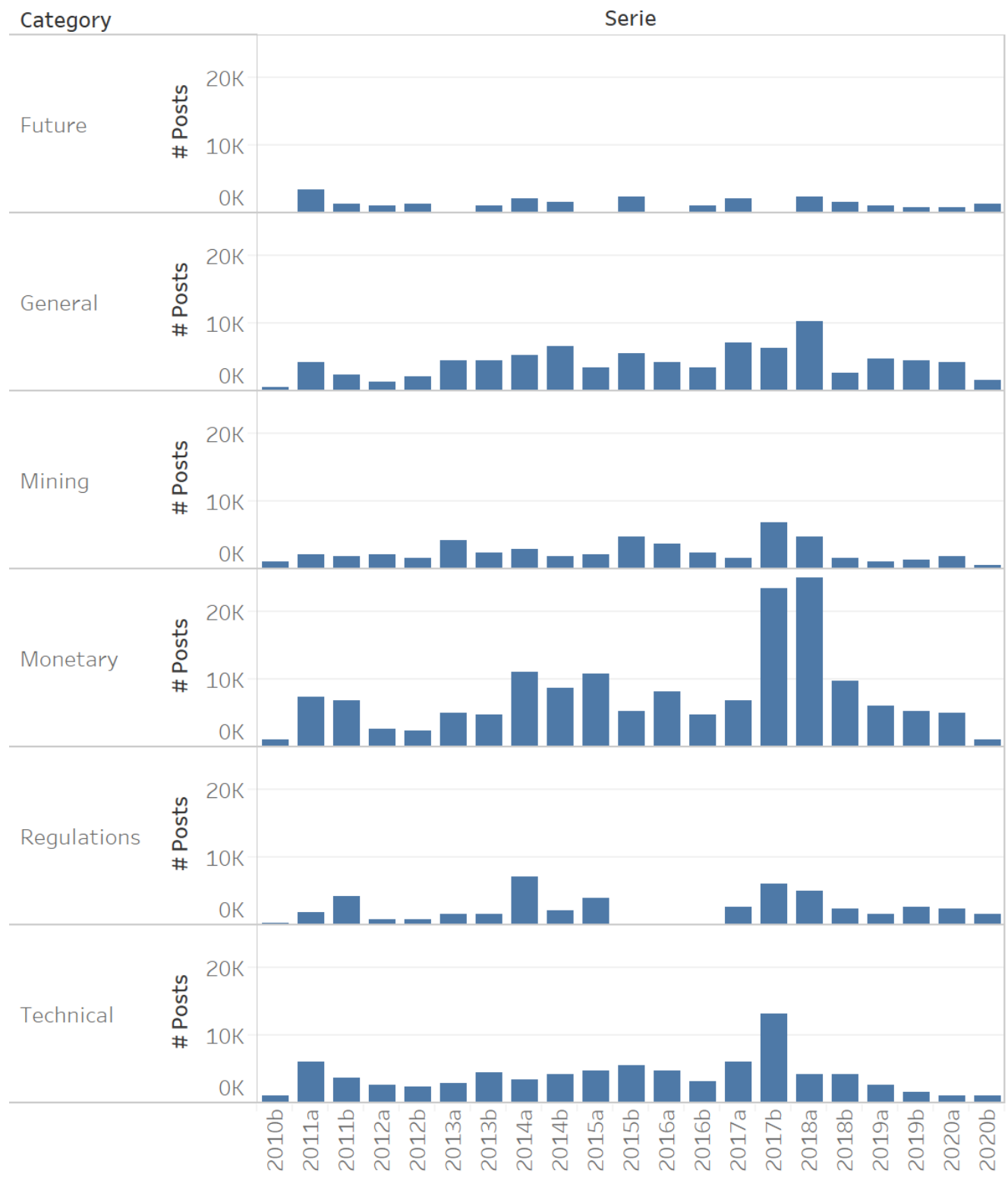

Figure 21: Number of posts in categories over the time. 


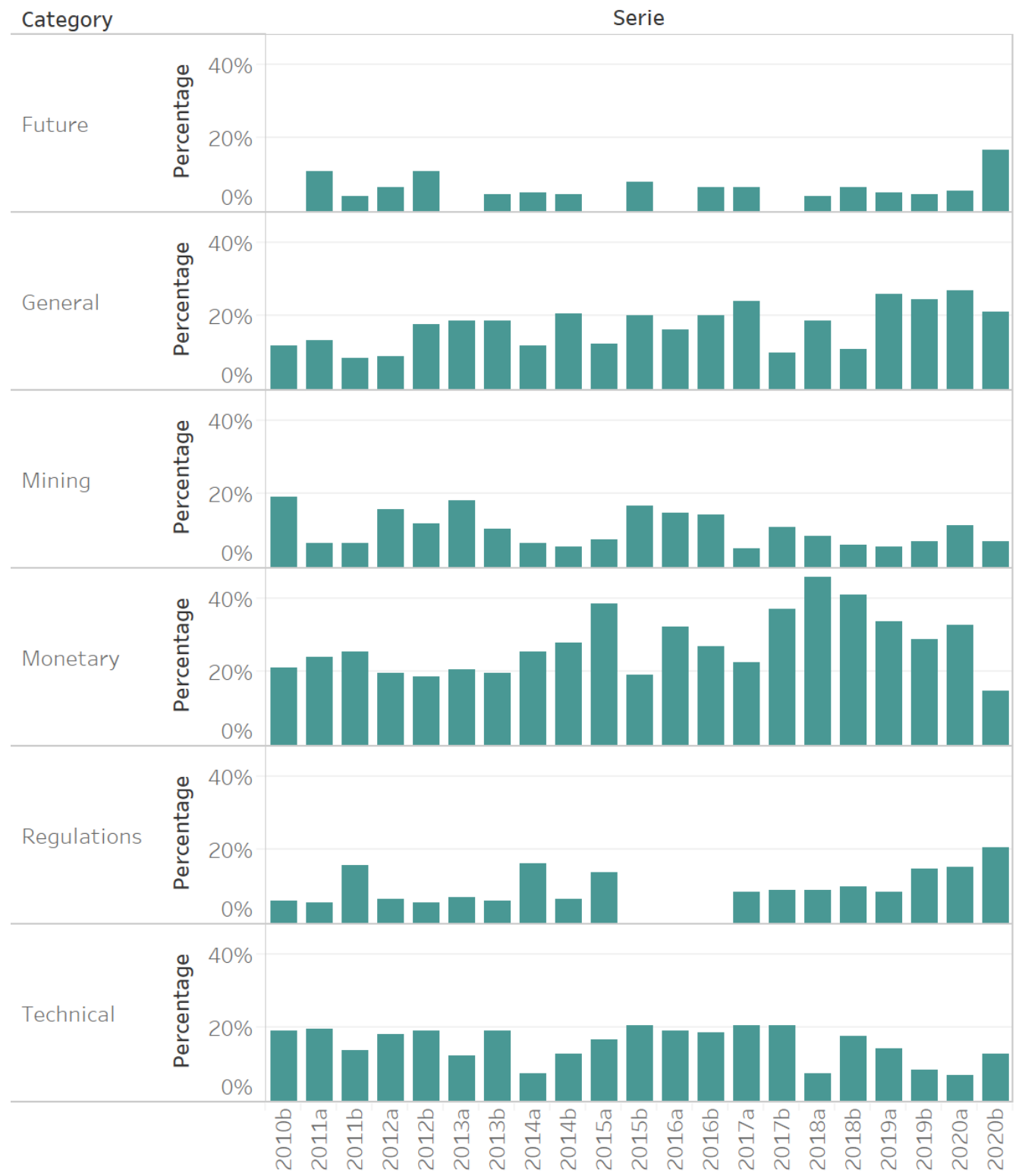

Figure 22: Percentage of categories for discussions over the time. 


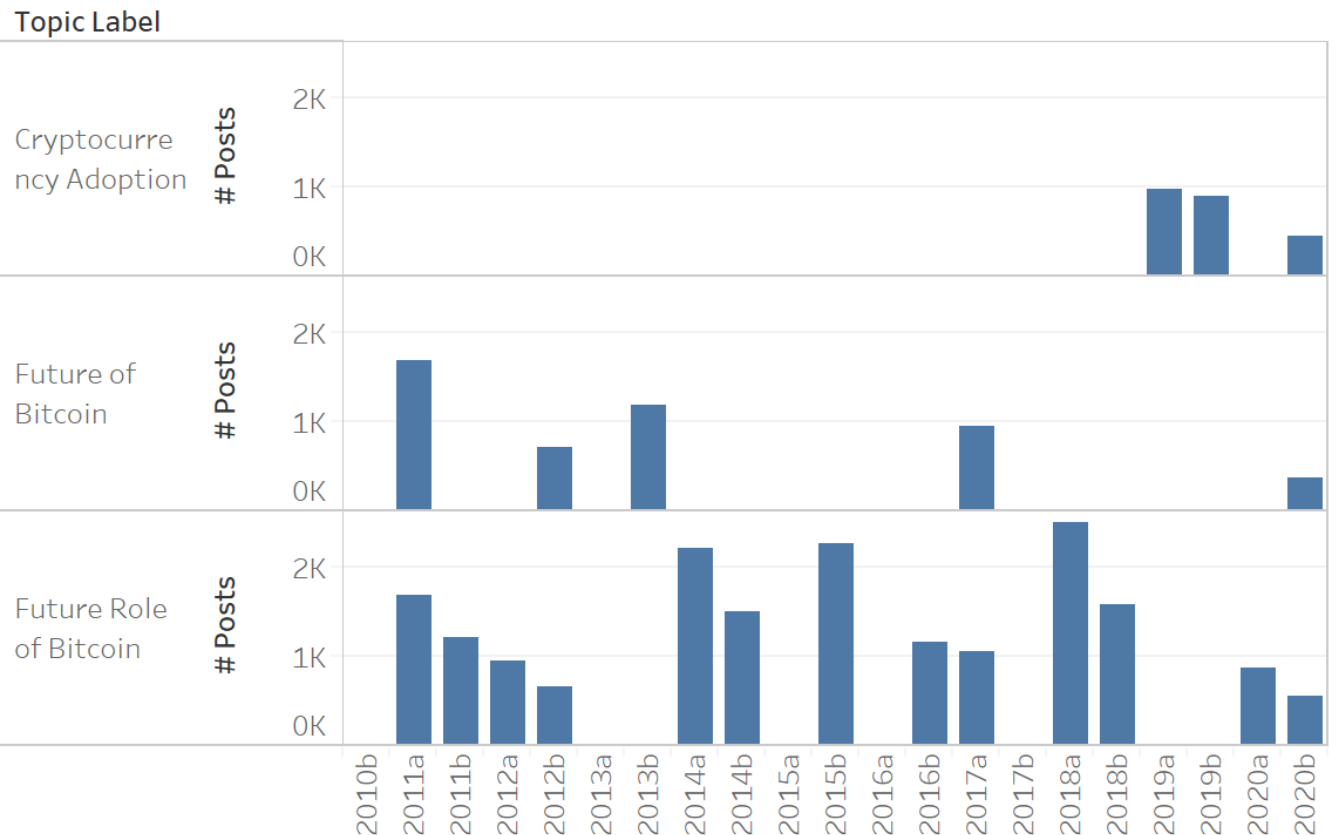

Figure 23: Discussion topics over the time in Future category.

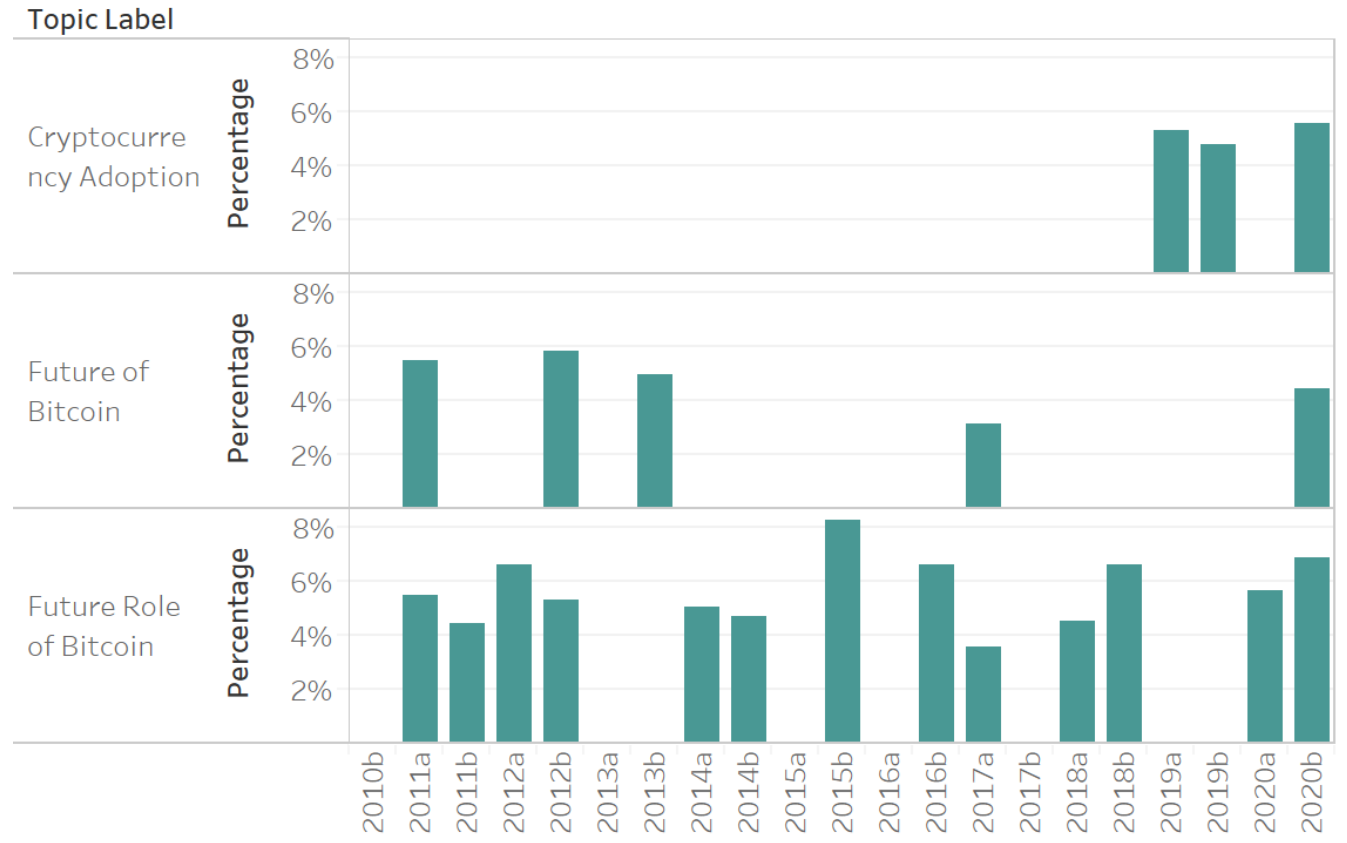

Figure 24: Distribution of topics over the time in Future category. 
Figure 26.

\subsubsection{Mining}

During the first 5 years usually both topics of this category which are related to transactions and requirements were present in discussions and frequently more than 10 percent of talks were related to these topics. In 2015 and 2016, the total number of discussions which were related to the topics exceeded 15 percent of the whole topics, however, from 2017b this value decreased to between 5 to 7 percent of the whole topics. The change trend of topics of this category has been presented in Figure 27 and Figure 28.

\subsubsection{Monetary}

This category is the most repetitive one among all the discussions of the forum. As we can see in Figure 29 and Figure 30, during the first years, a considerable part of the discussions were related to Currency and, little by little, Price as another topic that has emerged among the debates. From 2014 another subcategory that we labeled as Financial was created and even sometimes this subcategory overtook Currency in discussions. Price, on the other hand, showed up from 2017 and gradually it became the main subcategory of discussion in this category and in forum.

\section{Currency}

During the earliest years, the most frequent topic used to be "Payment with Bitcoin" that sometimes formed up to 15 percent of discussions in the forum. This topic gradually faded and was replaced by other topics in other subcategories. The next topic of this category is "Bitcoin Exchange Rate" that appeared in a larger number of datasets, and usually around 5 to 6 percent of debates are related to this topic. 


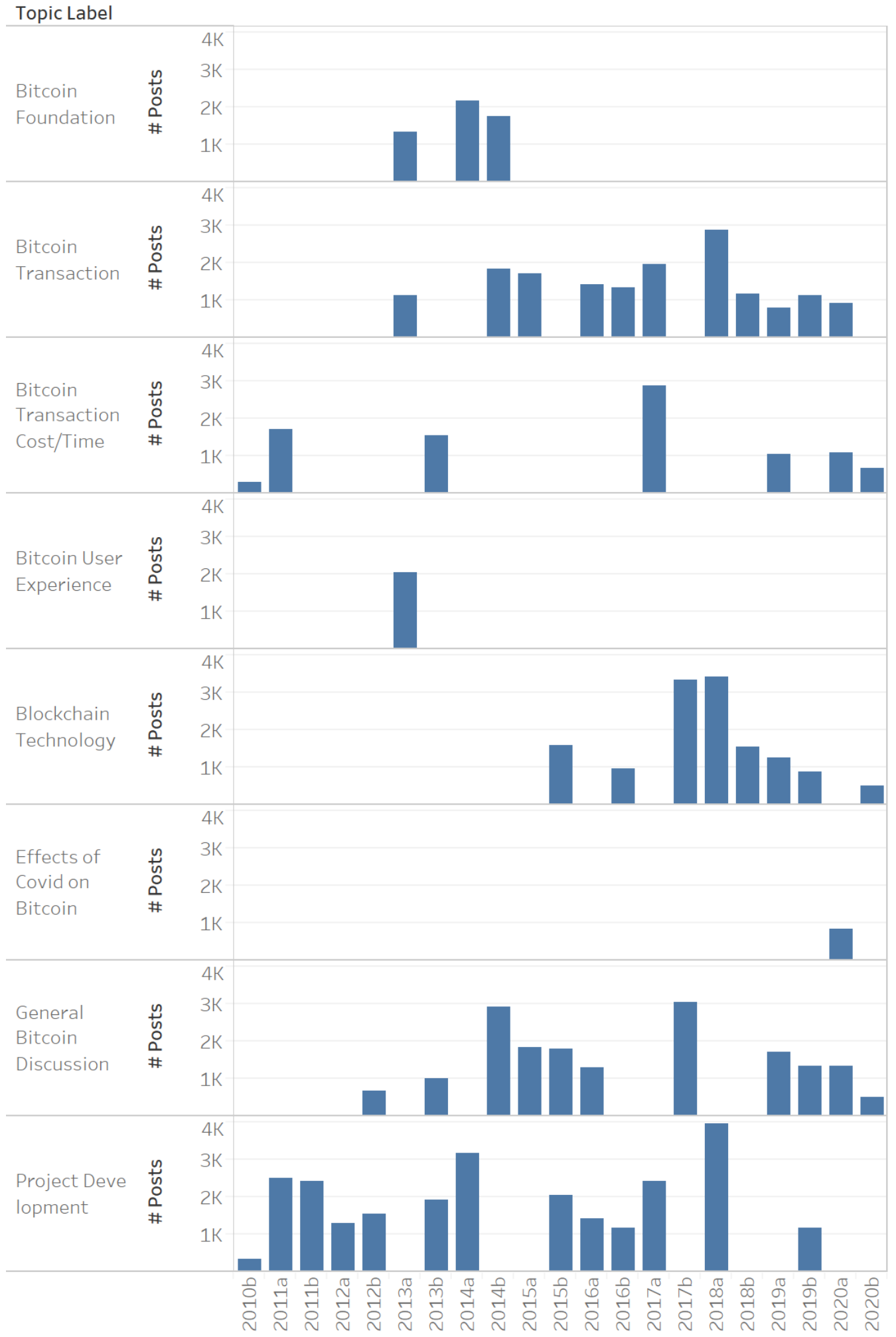

Figure 25: Discussion topics over the time in General category. 


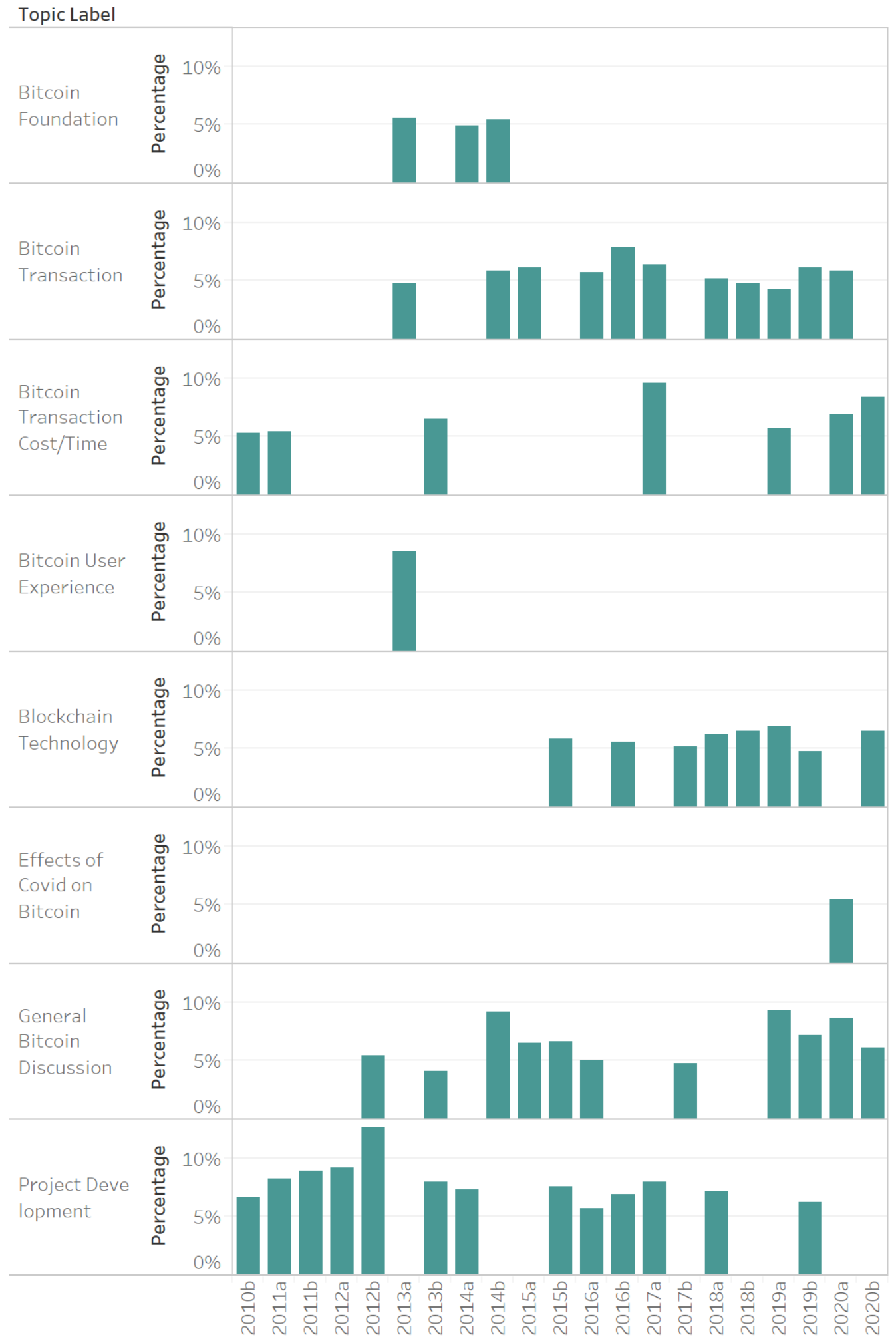

Figure 26: Distribution of topics over the time in General category. 


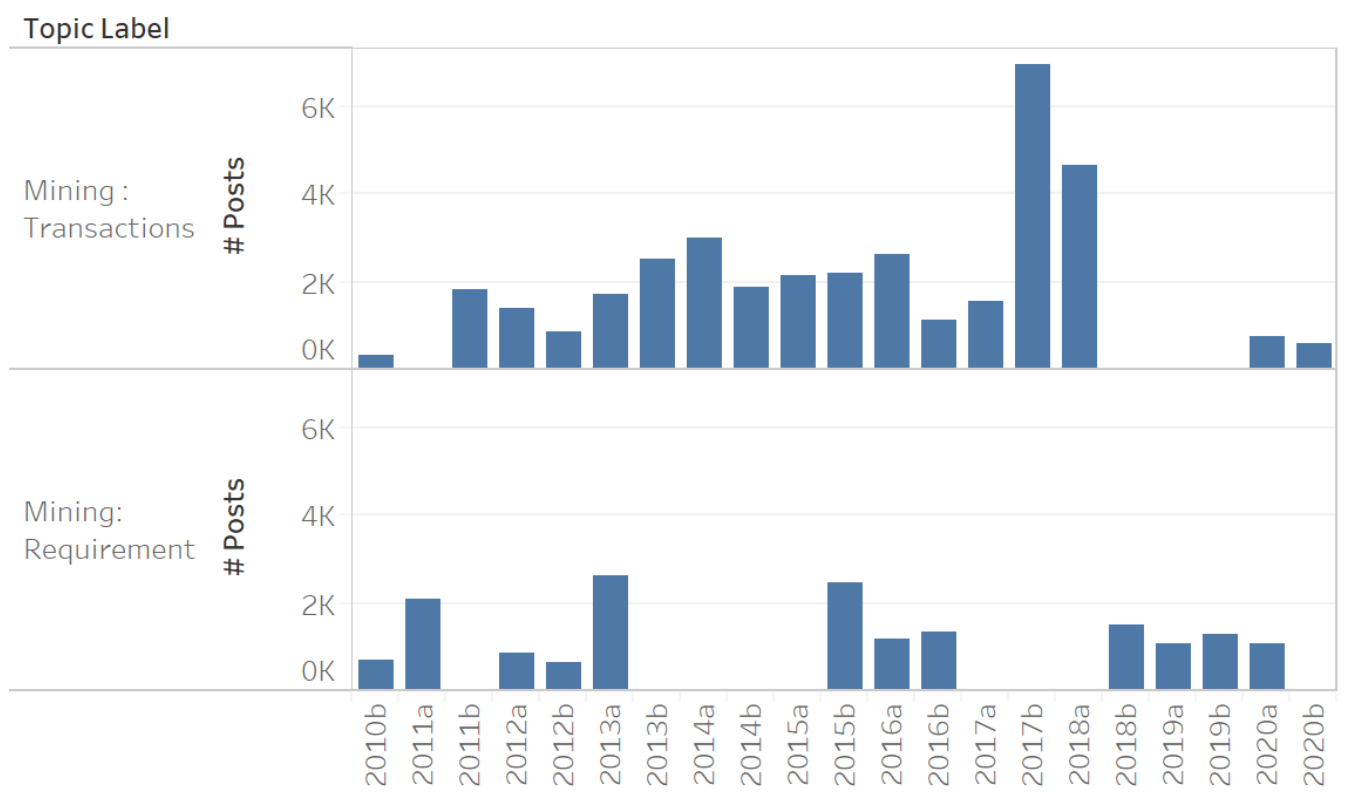

Figure 27: Discussion topics over the time in Mining category.

Topic Label

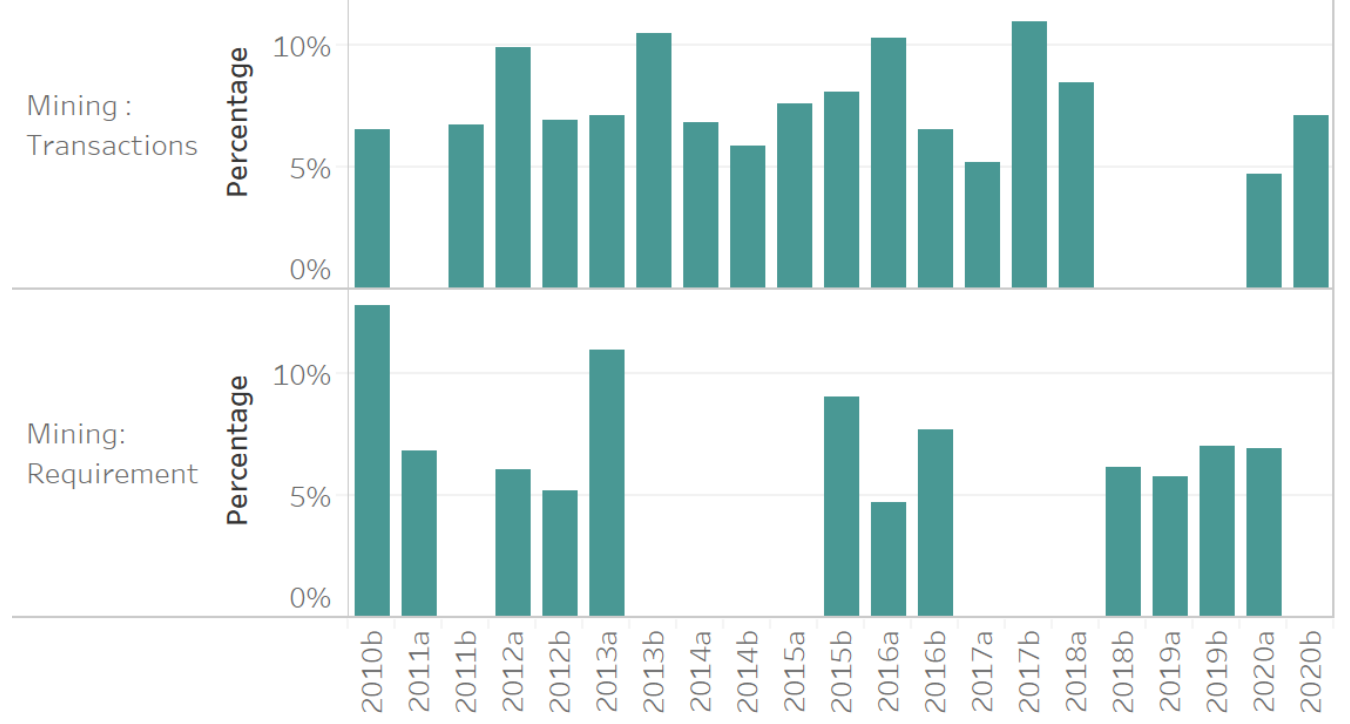

Figure 28: Distribution of topics over the time in Mining category. 


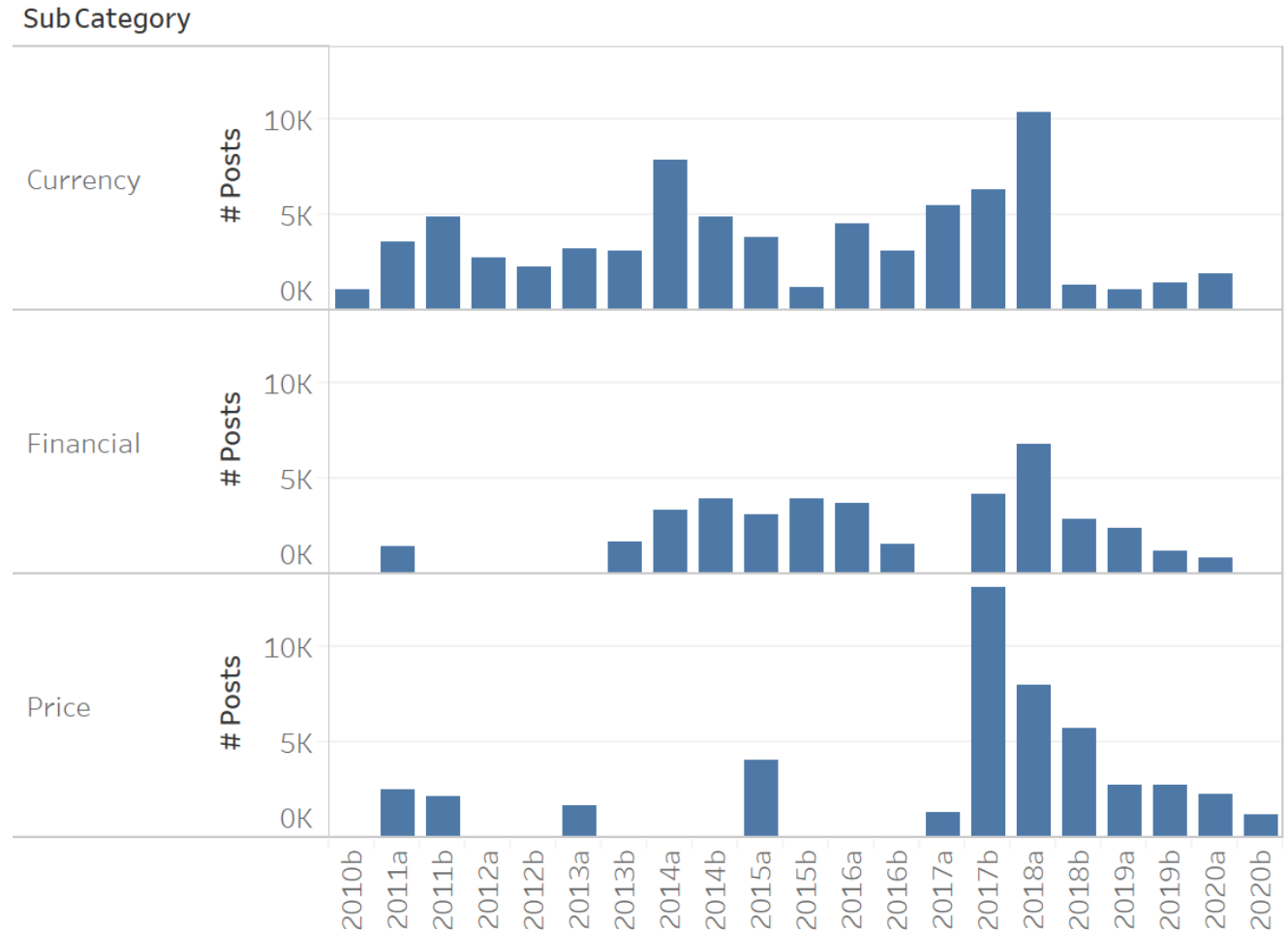

Figure 29: Discussion topics over the time in Monetary subcategory. 


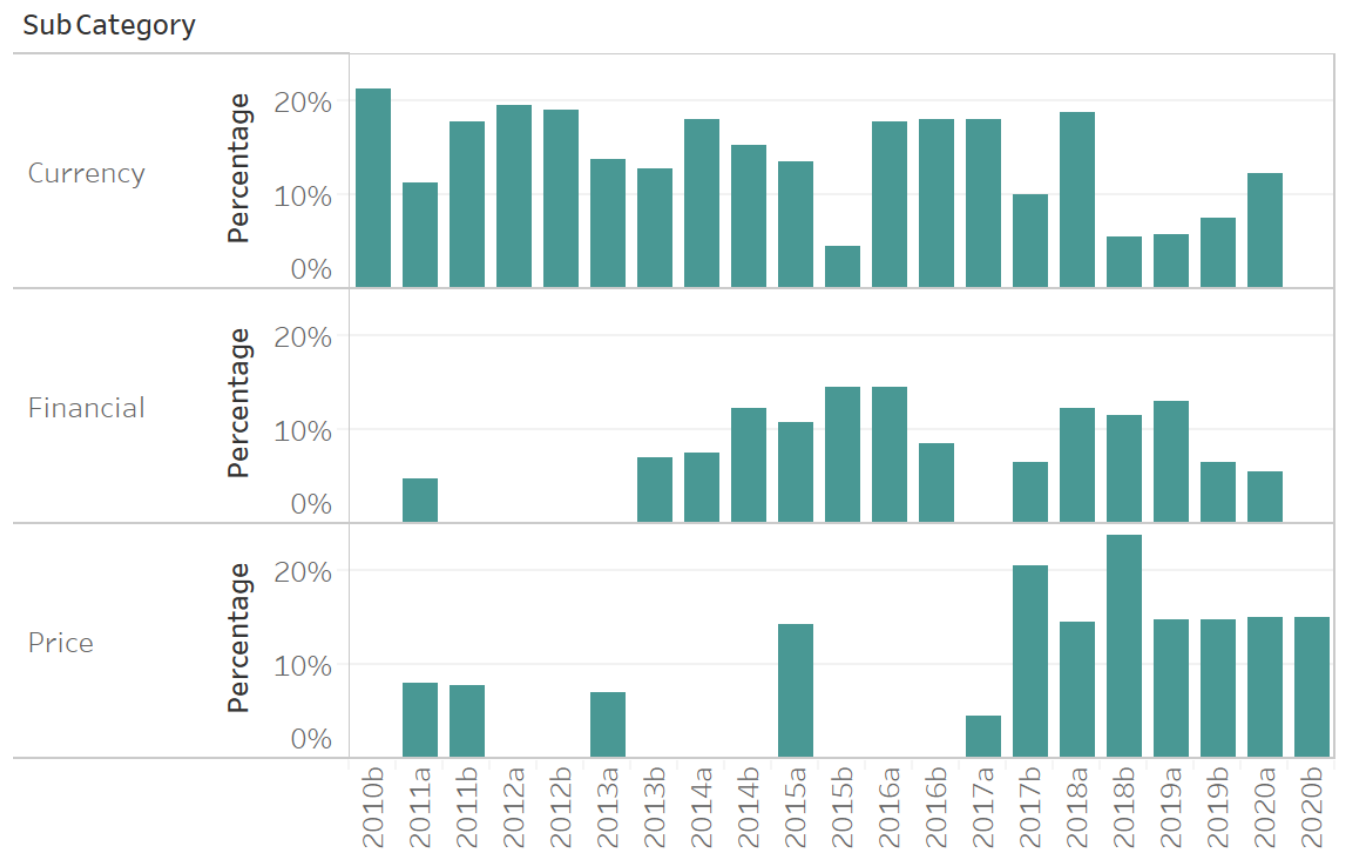

Figure 30: Distribution of topics over the time in Monetary subcategory.

The last topic in this category is "Bitcoin Alternatives" which has emerged in 2016 and in some periods forms up to 10 percent of talks. Figure 31 and Figure 32 present the change trend of topics of this category.

\section{Financial}

The largest number of discussions occurred in the "Financial Return" topic which formed up to 8 percent of debates between 2014 and 2018 in some specific years. This topic was replaced by "Bitcoin Trading" which has virtually the same proportion among discussions. "Effect of Bitcoin on Banking" has been created since 2013 and usually forms around 6 to 7 percent of conversations. The trend of changes in this category has been depicted in Figure 33 and Figure 34. 


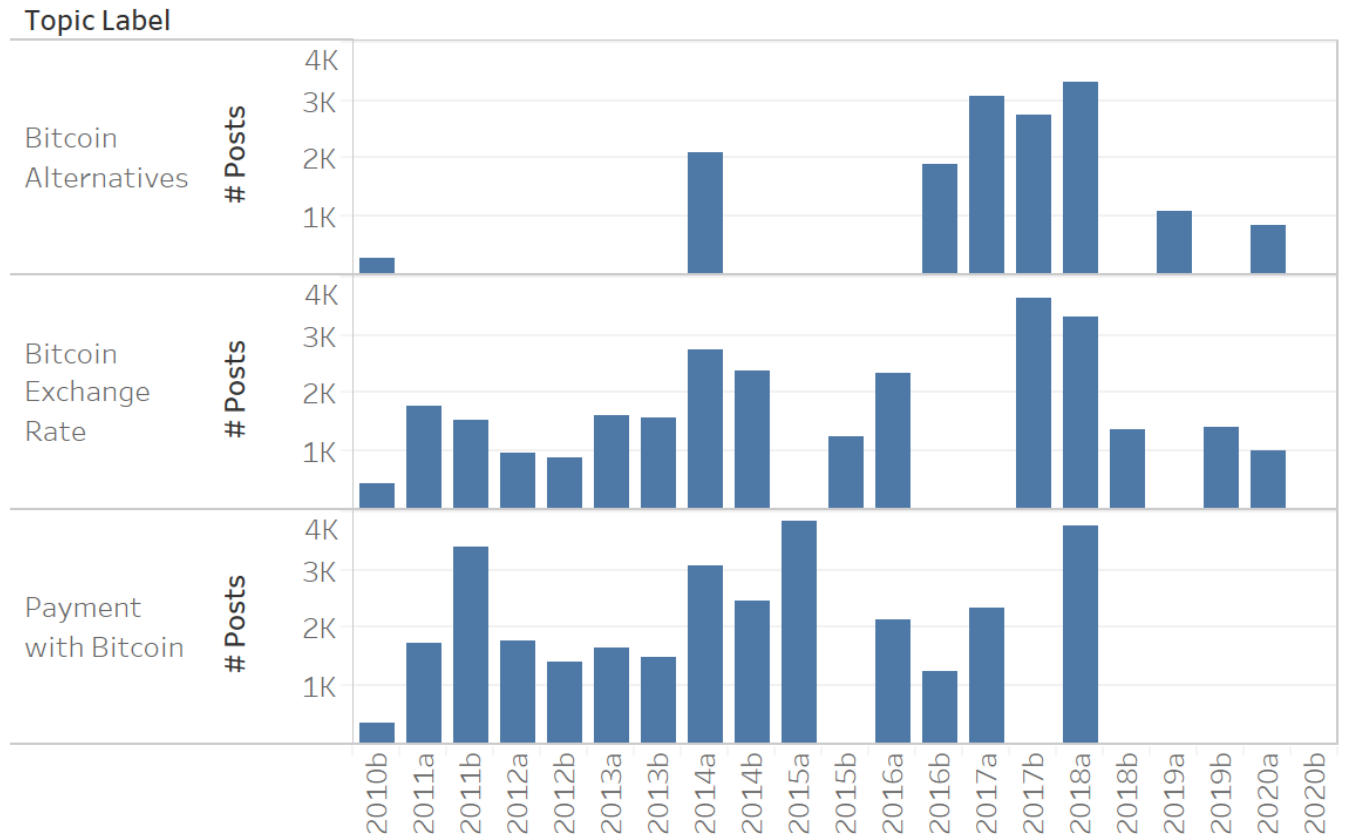

Figure 31: Discussion topics over the time in Currency subcategory.

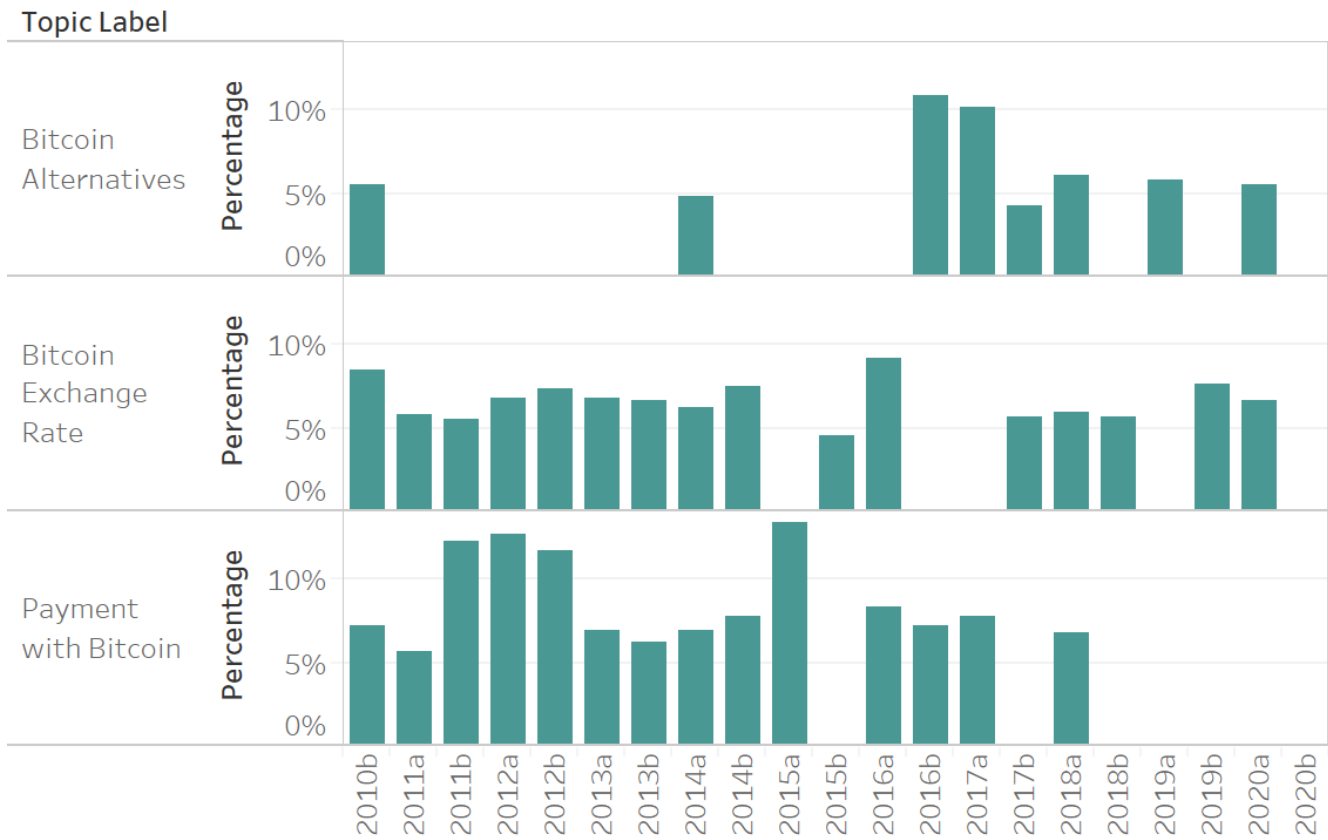

Figure 32: Distribution of topics over the time in Currency subcategory. 


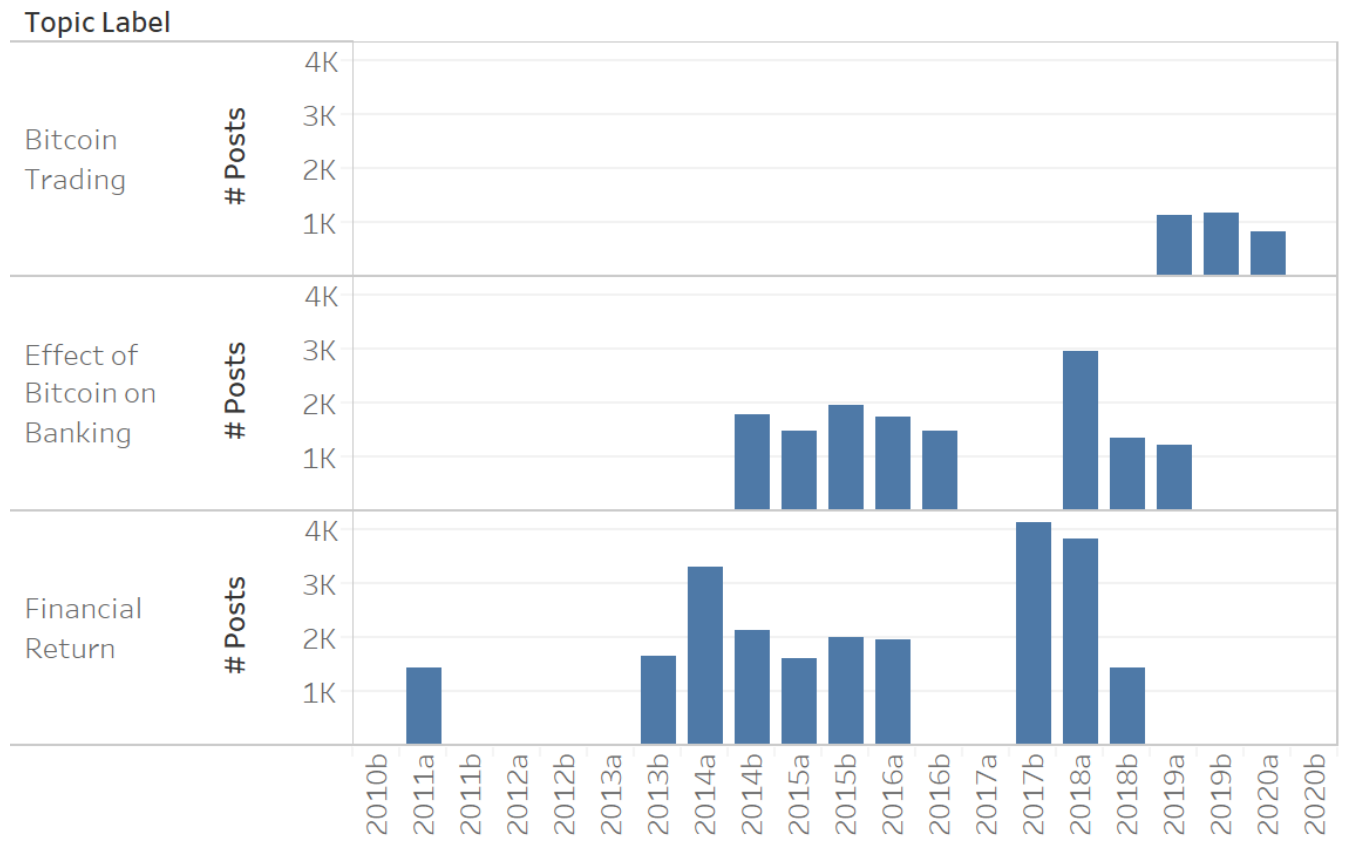

Figure 33: Discussion topics over the time in Financial subcategory.

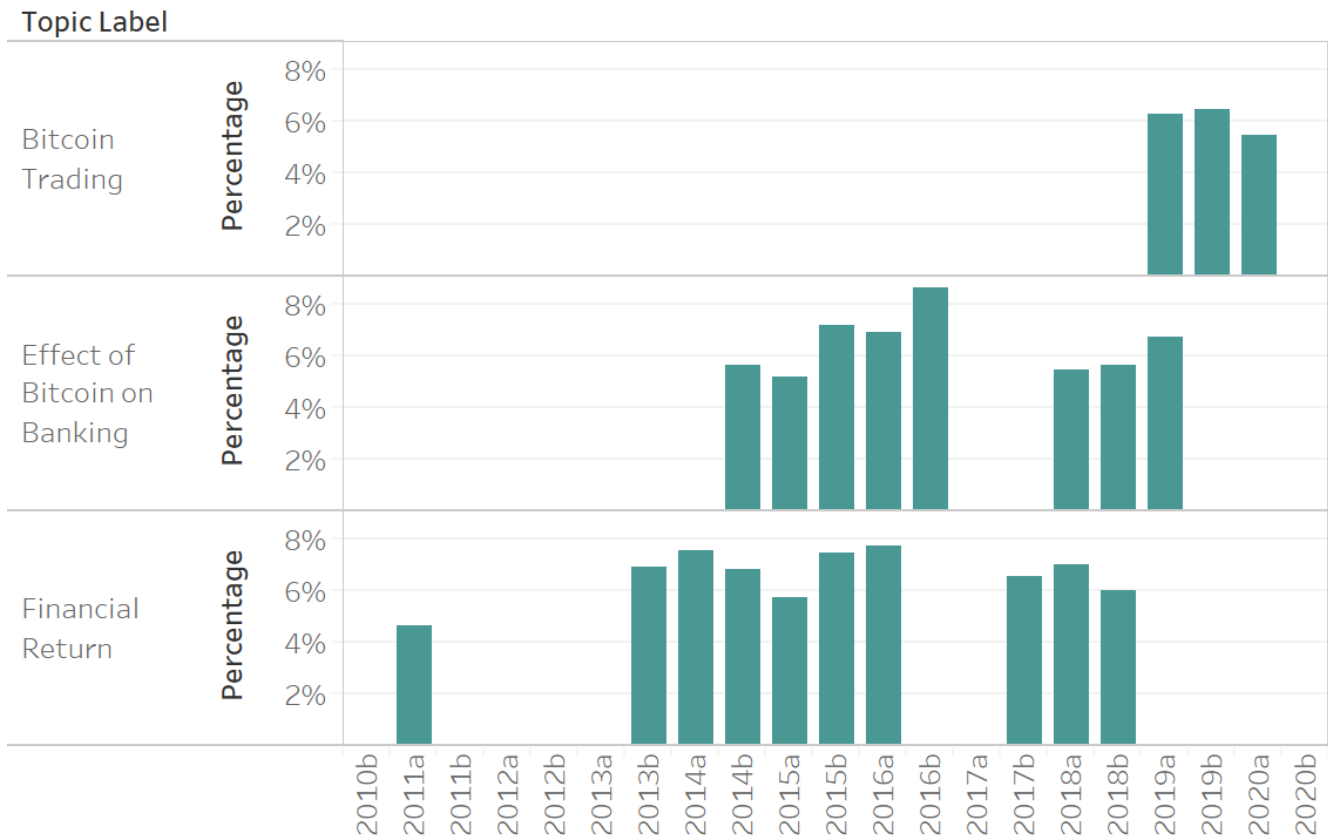

Figure 34: Distribution of topics over the time in Financial subcategory. 


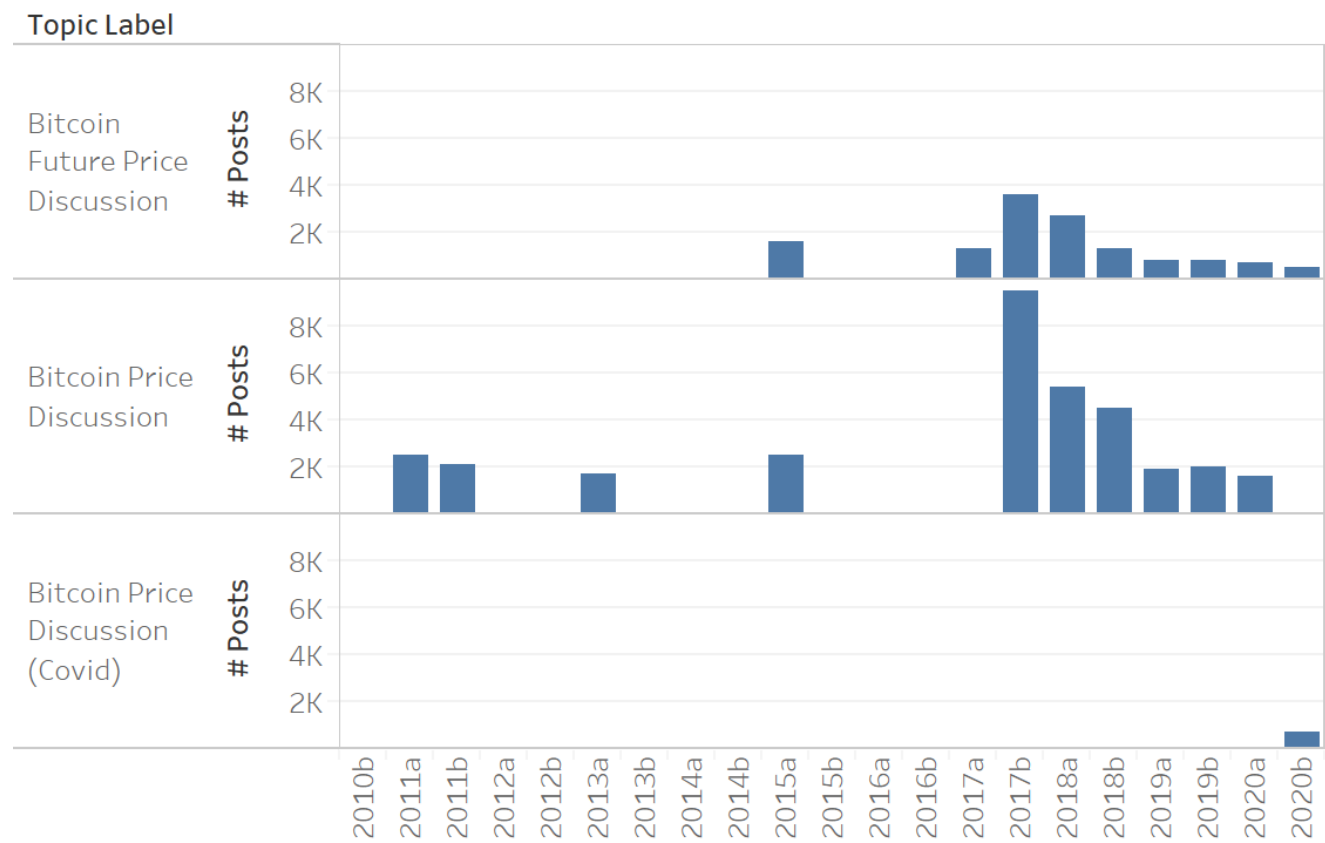

Figure 35: Discussion topics over the time in Price subcategory.

\section{Price}

"Bitcoin Price Discussion" can be seen in the discussions occasionally before 2017. From 2017 this topic appeared in conversations continuously. This topic, in turn, forms up to 10 to 20 percent of debates in the forum an counts as one of the most important ongoing topics. Simultaneously, from 2017 "Bitcoin Future Price Discussion" was added to the discussion topics of the forum and around 5 percent of discussions are related to this topic. Finally, we can find the impact of the emergence of COVID-19 that has resulted in the creation of a new topic, "Bitcoin Price Discussion (COVID)" which according to the similarity of its pattern and frequency to "Bitcoin Price Discussion", we can say that it is virtually a substitution for the mentioned topic. The change trend of discussion topics in this category can be found in Figure 35 and Figure 36 . 


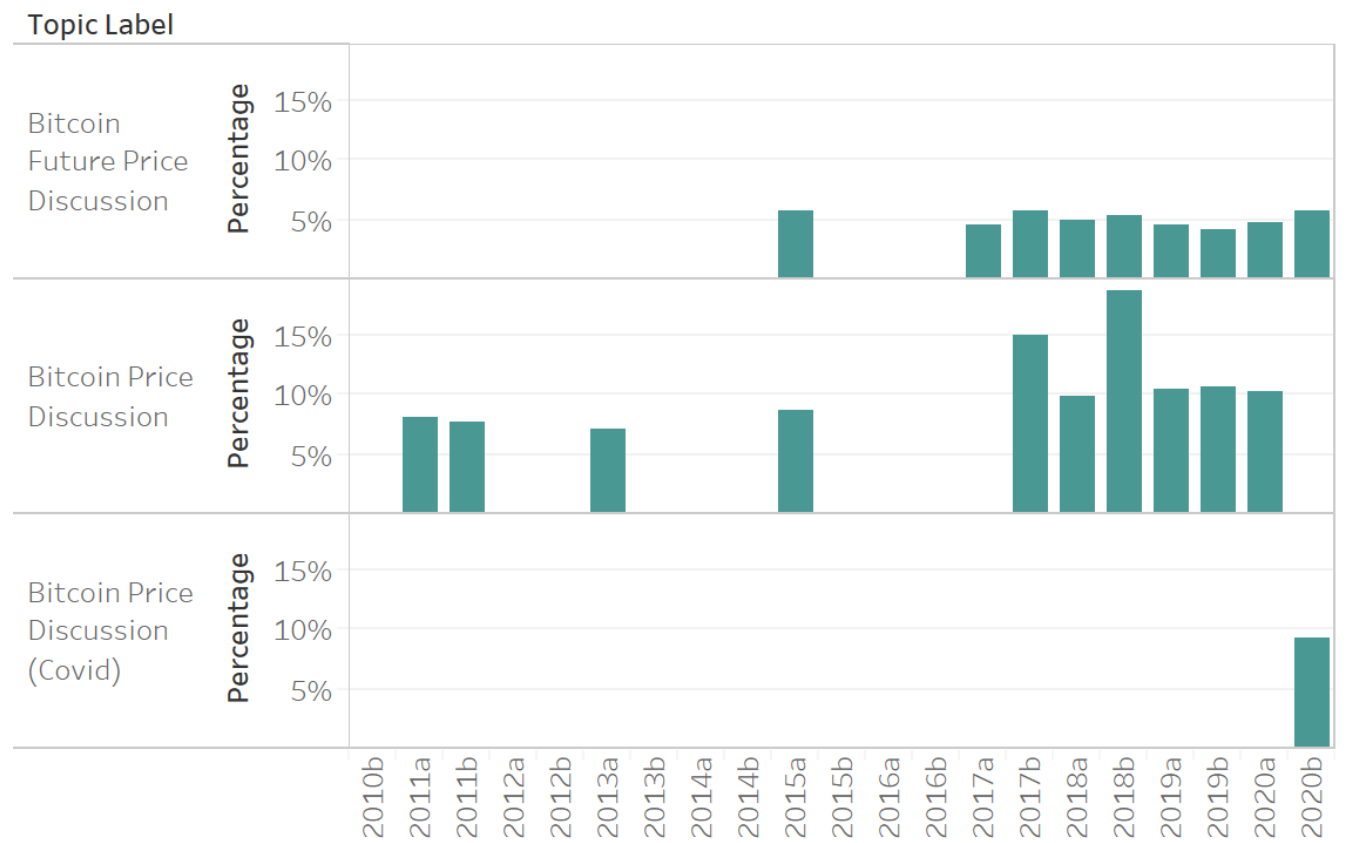

Figure 36: Distribution of topics over the time in Price subcategory.

\subsubsection{Regulations}

The major topic in this category is "Bitcoin Regulatory" which is a constant topic in the forum and expect for a 1.5 year period between 2015 and 2016, we can see this topic among all the discussion. This ongoing topic usually forms 5 to 10 percent of discussions in the forum. "Bitcoin scam" is a new topic in the forum which has emerged in 2019 and appears constantly in 7 to 8 percent of debates. "Bitcoin in Illegal Activity" is a topic that can be found occasionally in 4 sets and probably the number of discussions that are related to this topic varies according to the importance of the topic. For instance, it has the highest frequency in 2014a set. The change trend of discussion topics in this category can be found in Figure 37 and Figure 38. 


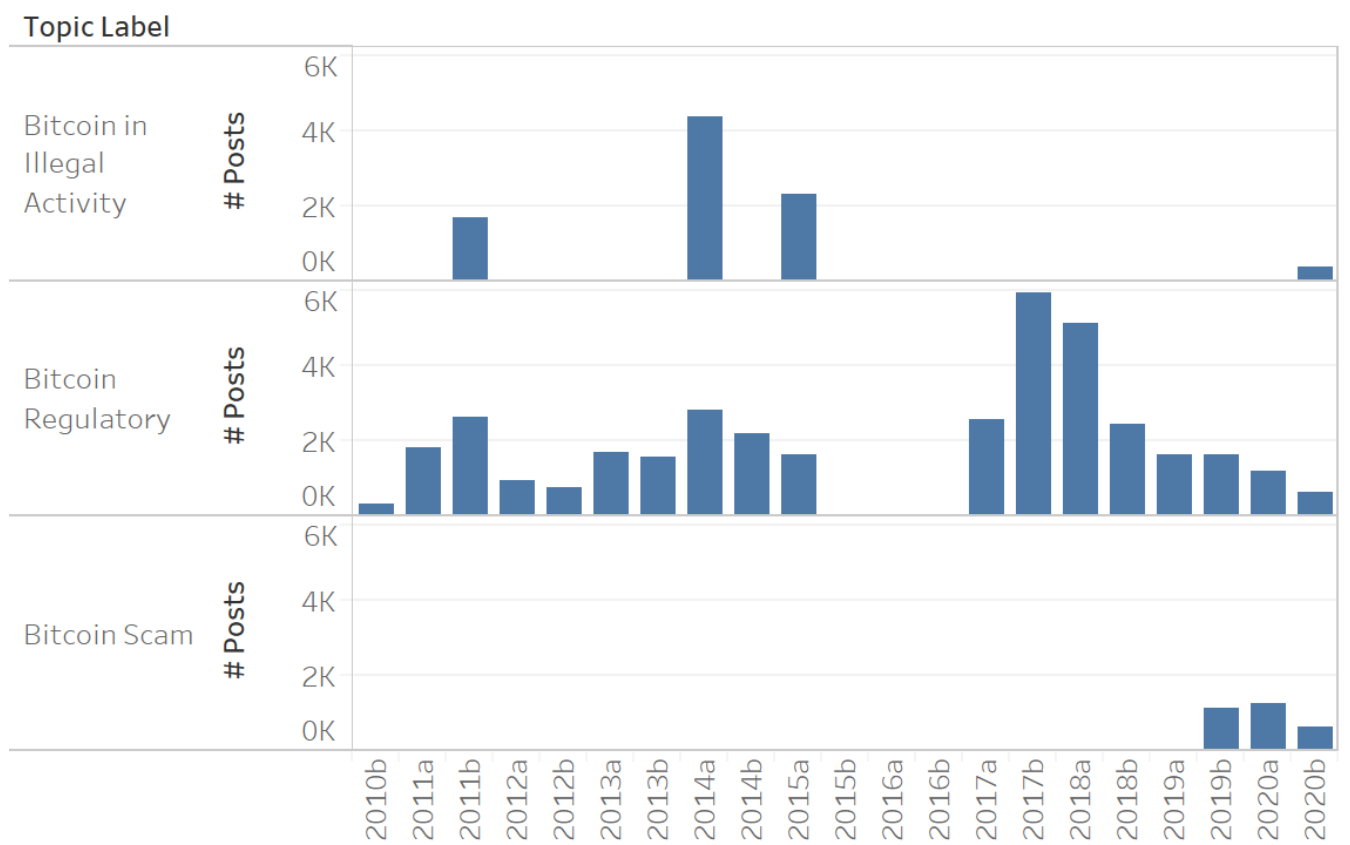

Figure 37: Discussion topics over the time in Regulations category.

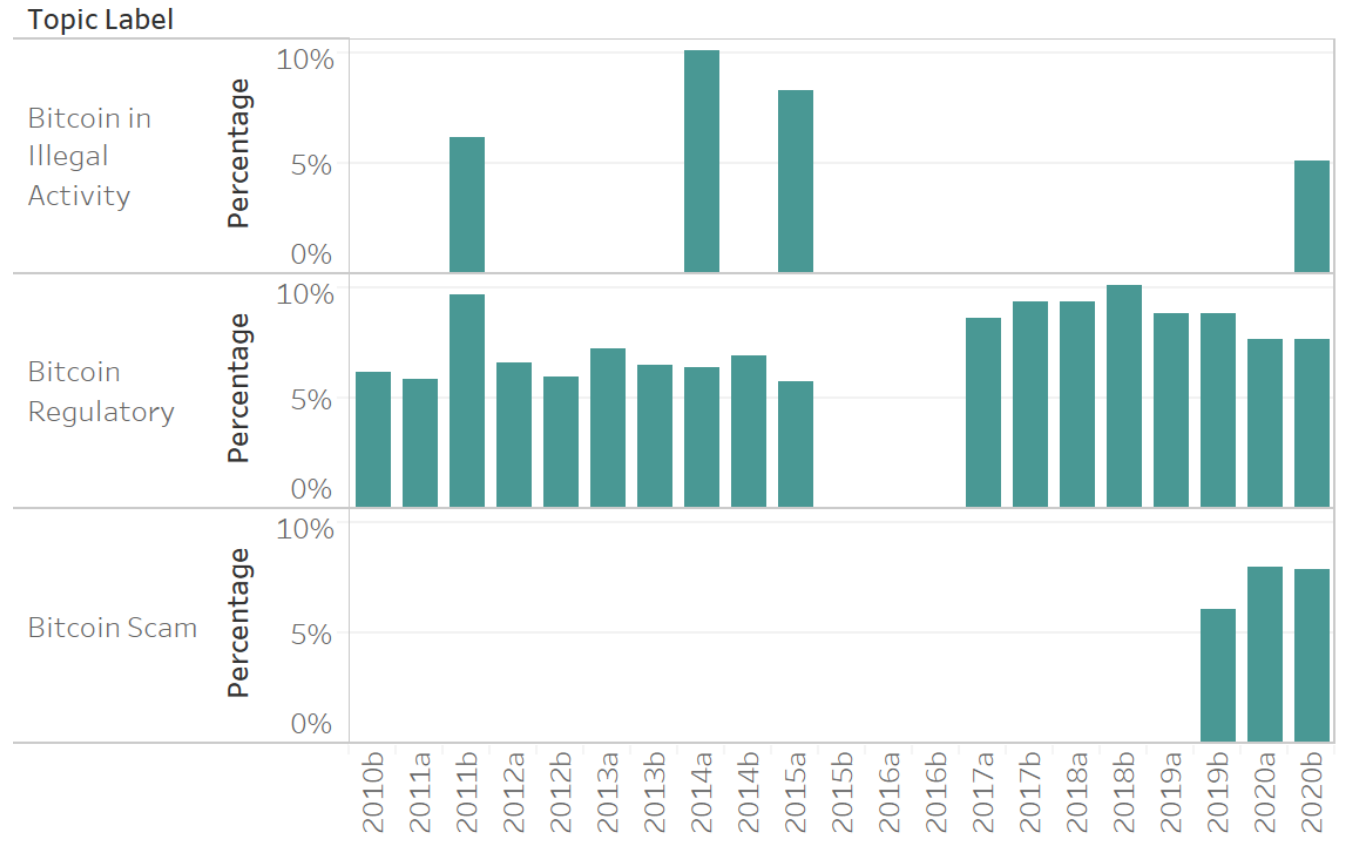

Figure 38: Distribution of topics over the time in Regulations category. 


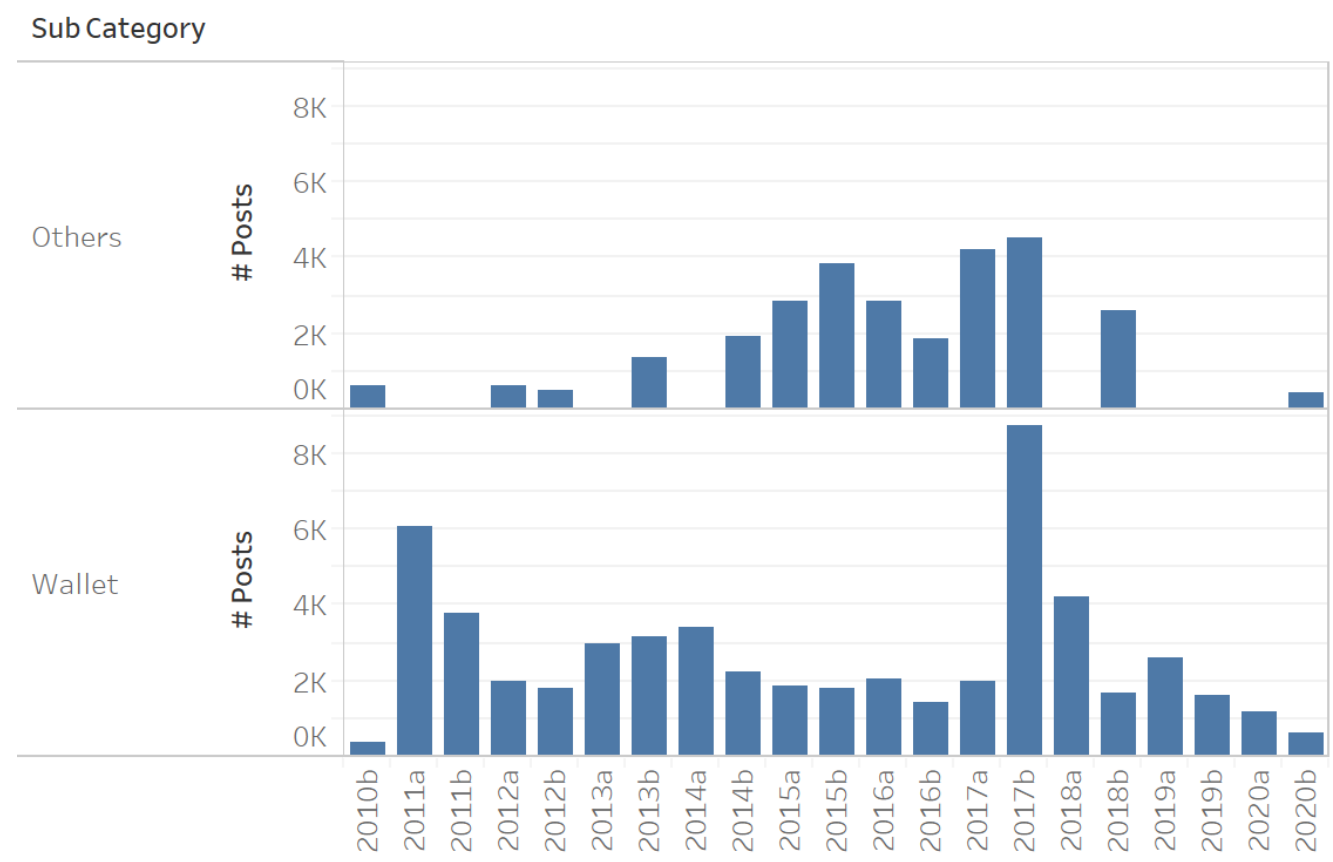

Figure 39: Discussion topics over the time in Technical subcategories.

\subsubsection{Technical}

In this category, Wallet appeared in all of the periods constantly, but Others can be found only in some periods occasionally. The trend of changes in subcategories has been depicted in Figure 39 and Figure 40. We should emphasize that the large number of posts which are placed in Others indicates their importance in discussions.

\section{Wallet}

This subcategory has two topics which are "User Security" and "Wallet Apps". User Security used to have a larger percentage of discussions during the earlier years, but in later periods it had a virtually constant percentage in the debates of the forum (around 7 to 8 percent). Similarly, "Wallet Apps" showed up in conversations occasionally with the frequency of around 5 to 8 percent. The change trend of discussion topics 


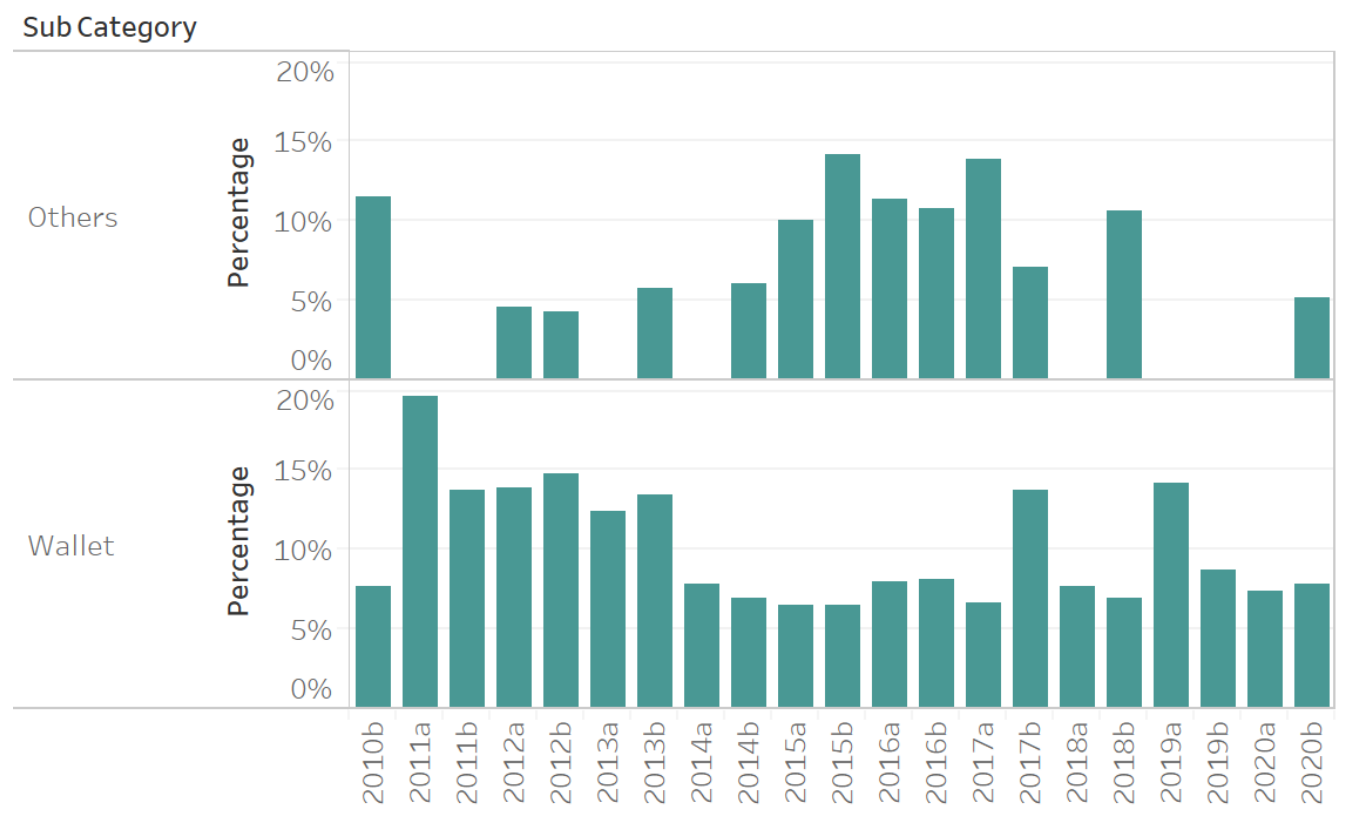

Figure 40: Distribution of topics over the time in Technical subcategories.

in this subcategory can be found in Figure 41 and Figure 42.

\section{Others}

The most frequent topic in this subsection is "Fork" which appeared in the forum only within a specific period (between 2015 and 2018) and disappeared after 2018. In 2017a set, this topic formed 14 percent of discussions. The other topics of this subsection showed up occasionally and formed up to 5 percent of the debates. Figure 43 and Figure 44 present the change trend of topics of this subcategory. 


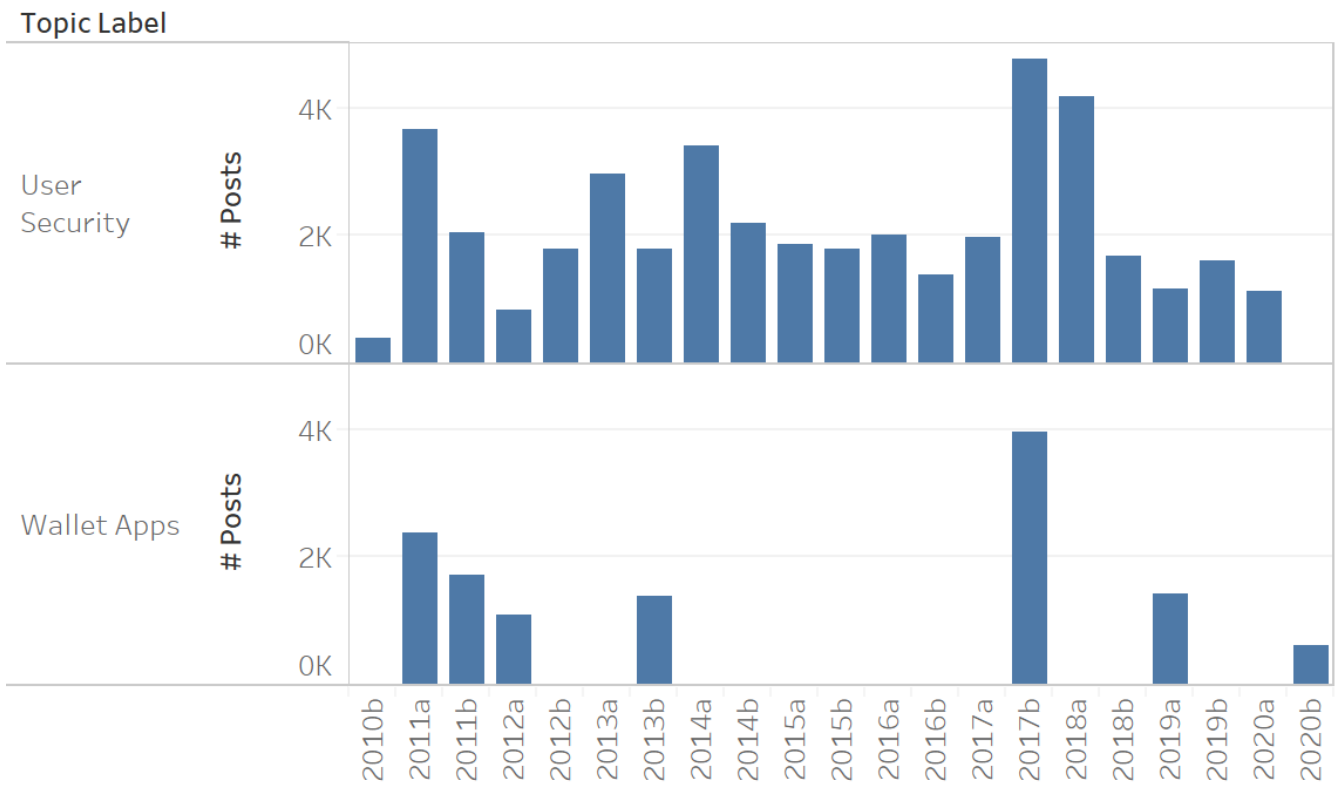

Figure 41: Discussion topics over the time in Wallet subcategory.

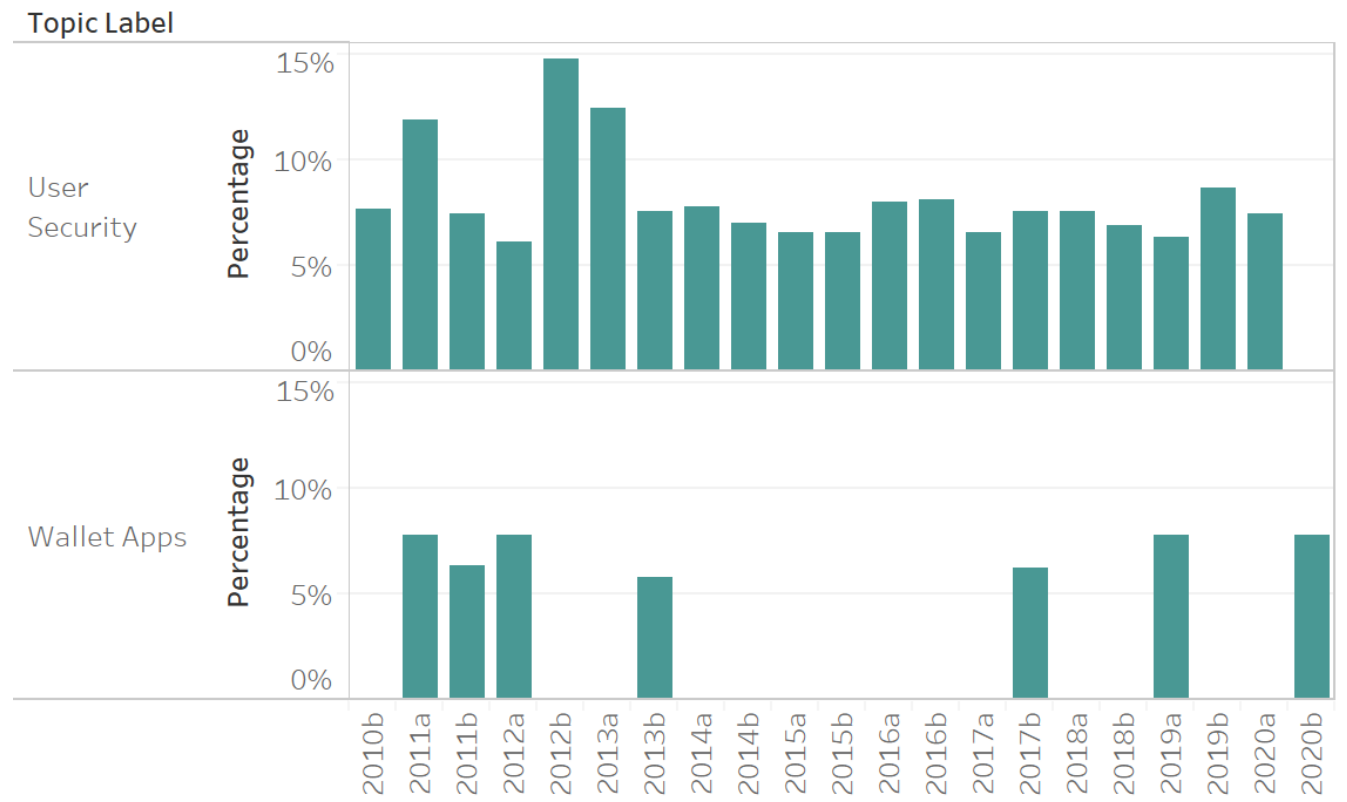

Figure 42: Distribution of topics over the time in Wallet subcategory. 


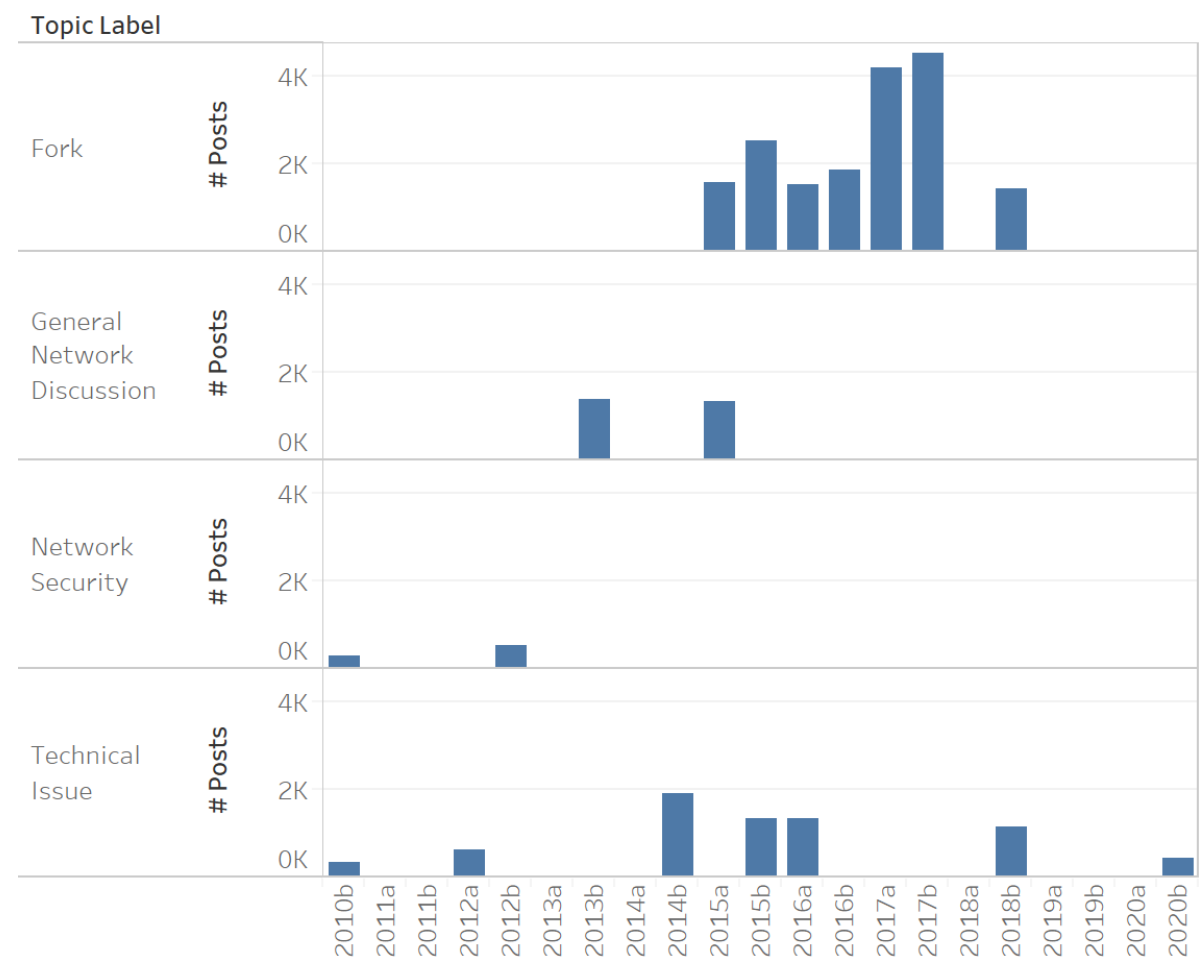

Figure 43: Discussion topics over the time in Others subcategory.

\section{Answer to RQ3:}

The number of posts in the forum has been considerably varied during different time periods. However, over the past two years, the overall number of posts has decreased in the forum and the number of posts in this period is less than half of the number of posts in 2017 and 2018. The highest growth of post numbers happened up to 2018 in Monetary topics, and it is worth mentioning that the discussion topics in this category have changed from general discussions to price-oriented talks. The highest decrease in the discussions is related to Technical and Mining categories. Furthermore, the percent of discussions about Regulations has increased over the past years compared to previous years. 


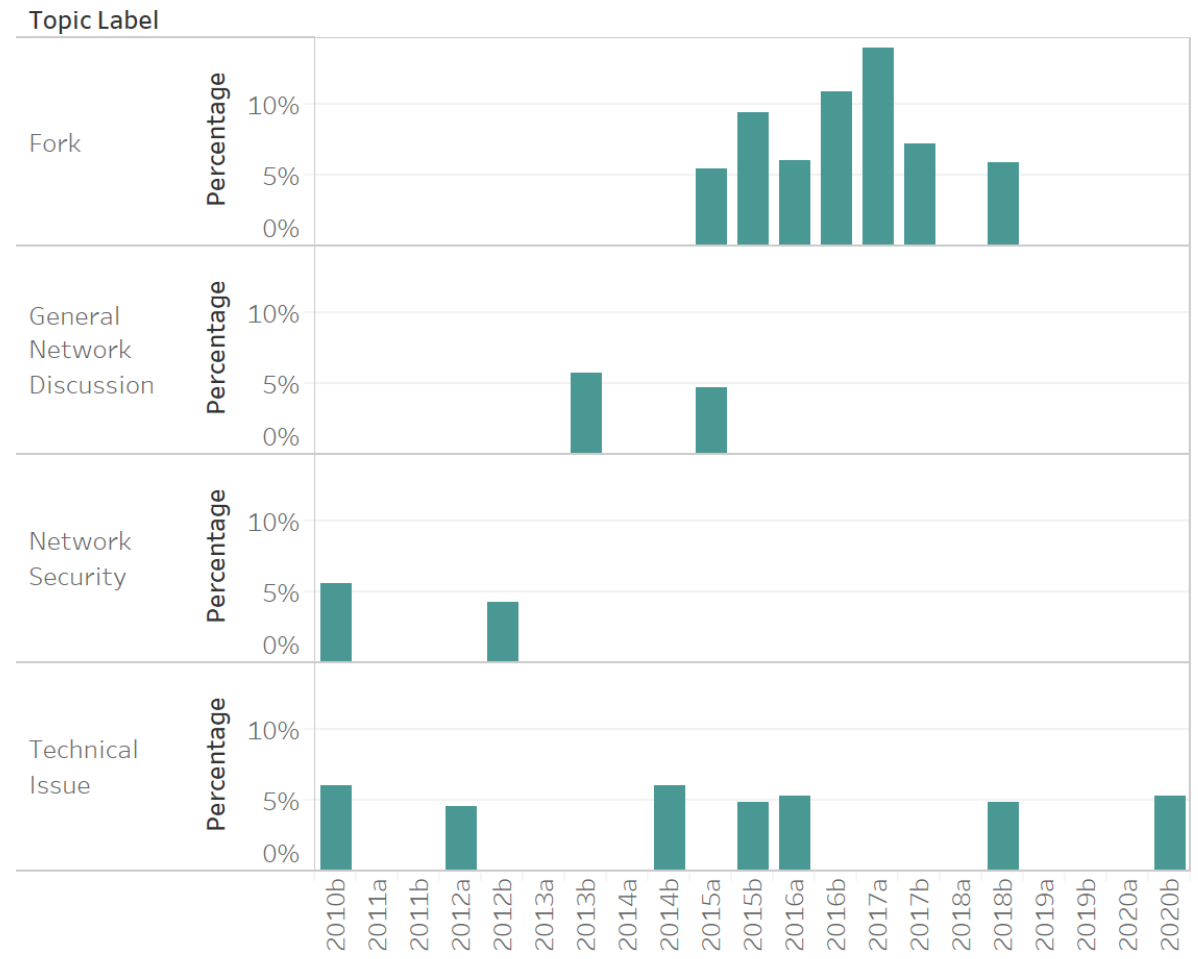

Figure 44: Distribution of topics over the time in Others subcategory. 


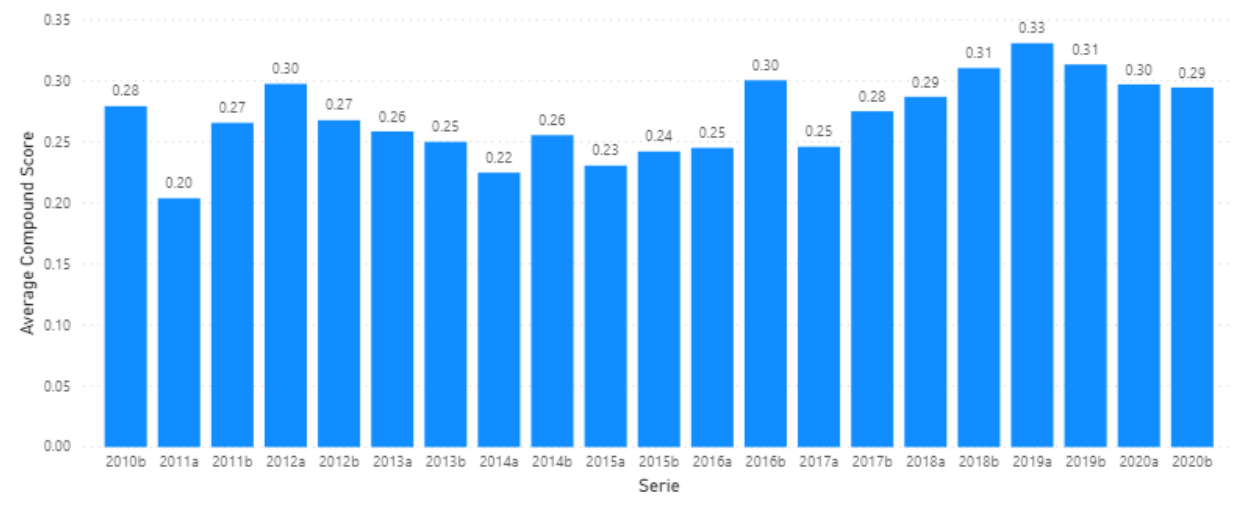

Figure 45: Average sentiment compound score of the total posts over the time.

\subsection{RQ4: What is the sentiment polarity of dis- cussions in each topic?}

In this question, we evaluated the sentiment polarity of posts in the forum. With a quick glance in Figure 45, we can see that the overall sentiment weight of posts in all years was positive which indicates a general positive tendency in discussions. However, as Figure 46 shows, we can find that the percentage of neutral posts has reduced from around 20 percent to around 10 percent which implies the creation of sentiment polarity among the posts. Respectively, we can find that average compound score in both positive and negative categories has increased gradually.

If we want to evaluate polarity in different categories, we can see in Figure 47 that the situation varies in different categories, for instance, the volume of polarity in Mining and Regulations is less than other categories. We next present the answer for this question with more detail for every category just like what we did for the previous questions. We describe the details using two charts: similar to Figure 47 , each bar chart represents the average compound score for each topic and a stack bar chart similar to Figure 46 represents the percentage of positive, negative and neutral posts in each category. In addition, we present the detail tables of results for each 


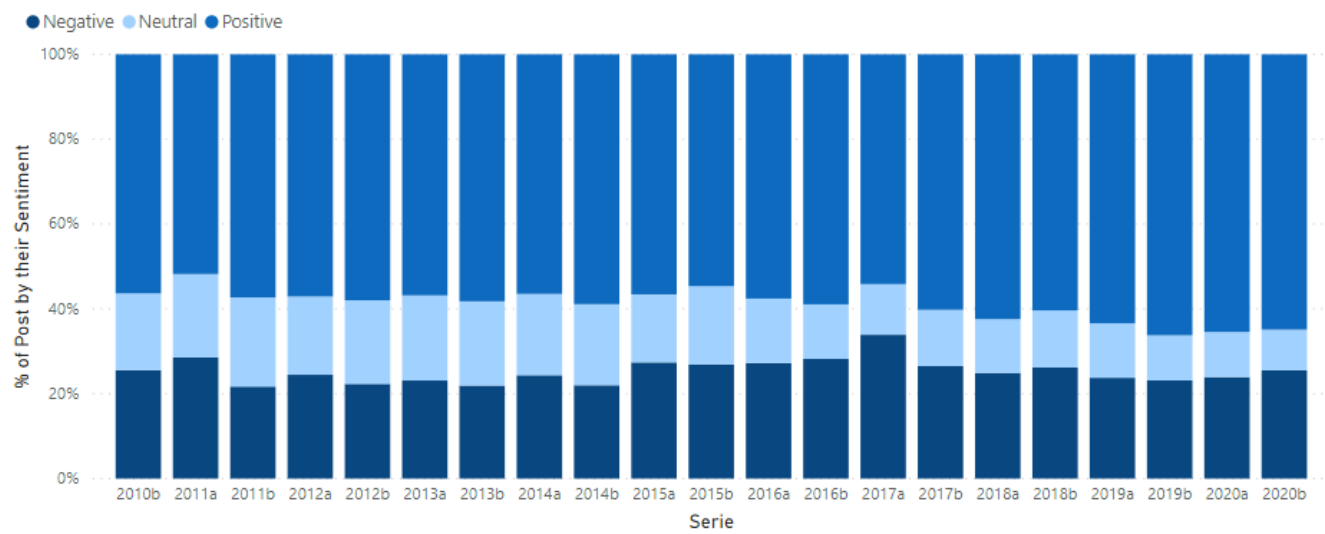

Figure 46: Distribution of positive/negative posts over the time.

topic in Appendix A.

\subsubsection{Future}

The considerable point in this category is that the amount of positive posts has increased noticeably from 2017 and in some periods, we can see the compound score of 54 percent and higher in the discussions of this category. "Cryptocurrency Adoption" has usually a high positive score which indicates a general positive perception about this topic on the forum. "Future Role of Bitcoin" has experienced a high fluctuation in score. Except for the last two or three years, the value of compound score for this topic varied in the range of 0.12 to 0.37 . However, during the latest years the compound score of the posts in this topic has risen to 0.4 and 0.5 . Proponent to the increase in the value of compound score, the percentage of positive posts has already increased during the latest years (around 15 percent) and the greatest change is the tendency towards having higher positive compound scores. The compound score of "Future of Bitcoin" is almost constant with a tiny fluctuation around 0.25. The information about the average compound score of each topic of this category and the distribution of positive, negative and neutral posts are depicted in Figure 48 and 


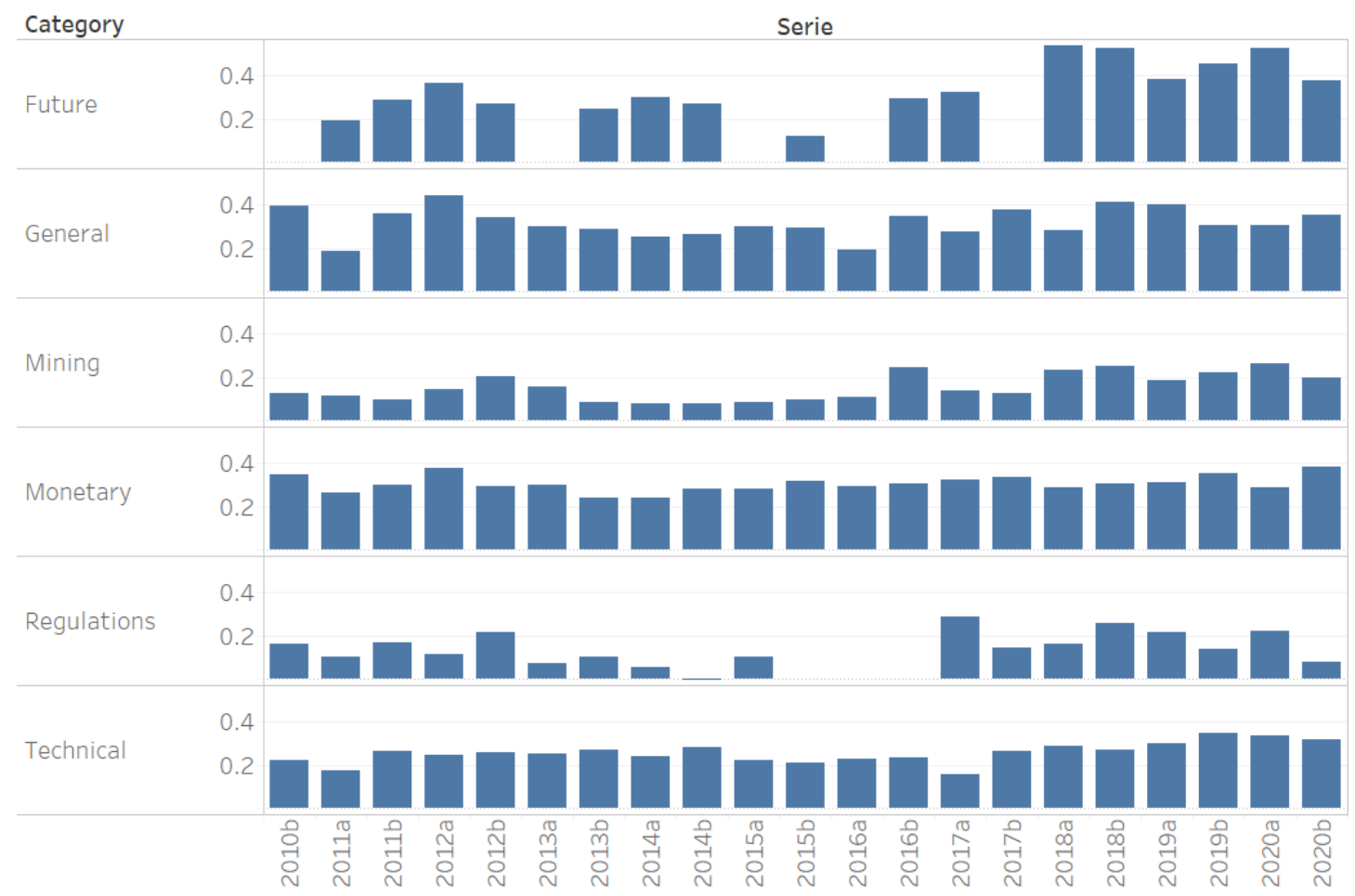

Figure 47: Compound score of posts in categories over the time.

Figure 49 .

\subsubsection{General}

We can find different polarity scores for different topics of this category. Except for "Blockchain Technology" in which the general perception is positive, we can see diverse sentiment polarity for the rest of the topics. For instance, the range of compound score in "Bitcoin Transaction Cost/Time" varies between 0.03 and 0.4 which indicates the diversity of sentiment polarity in this topic. The percentage of positive, negative and neutral posts in this category is similar to what we find about all of the posts and gradually, the number of neutral posts has decreased and the number of positive posts has increased correspondingly. The trend of changes in this category has been depicted in Figure 50 and Figure 51 . 


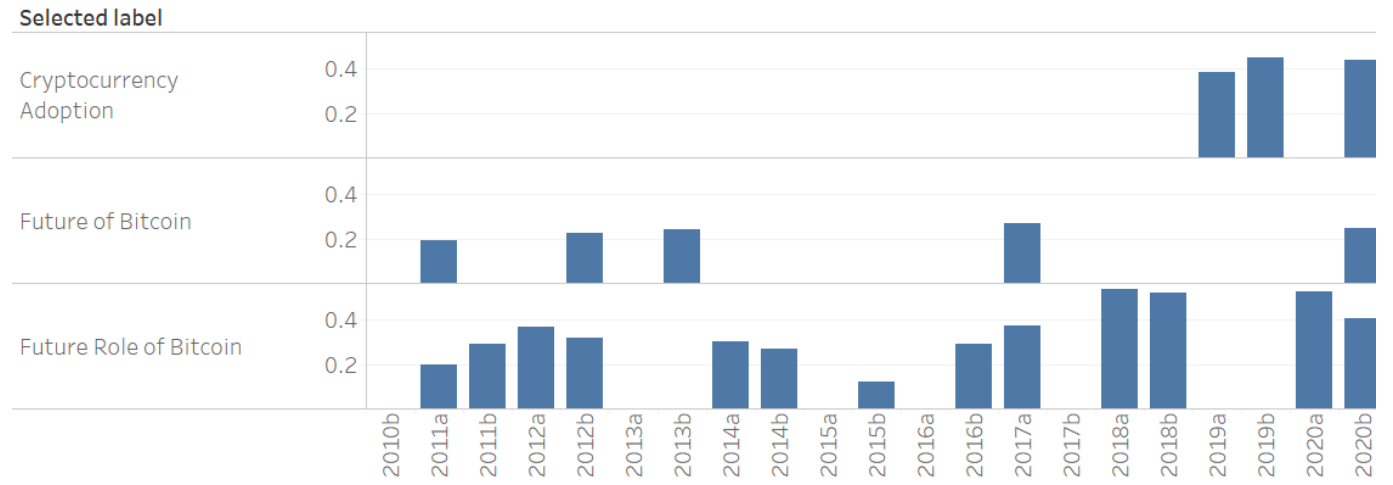

Figure 48: Compound score of topics in Future category over the time.

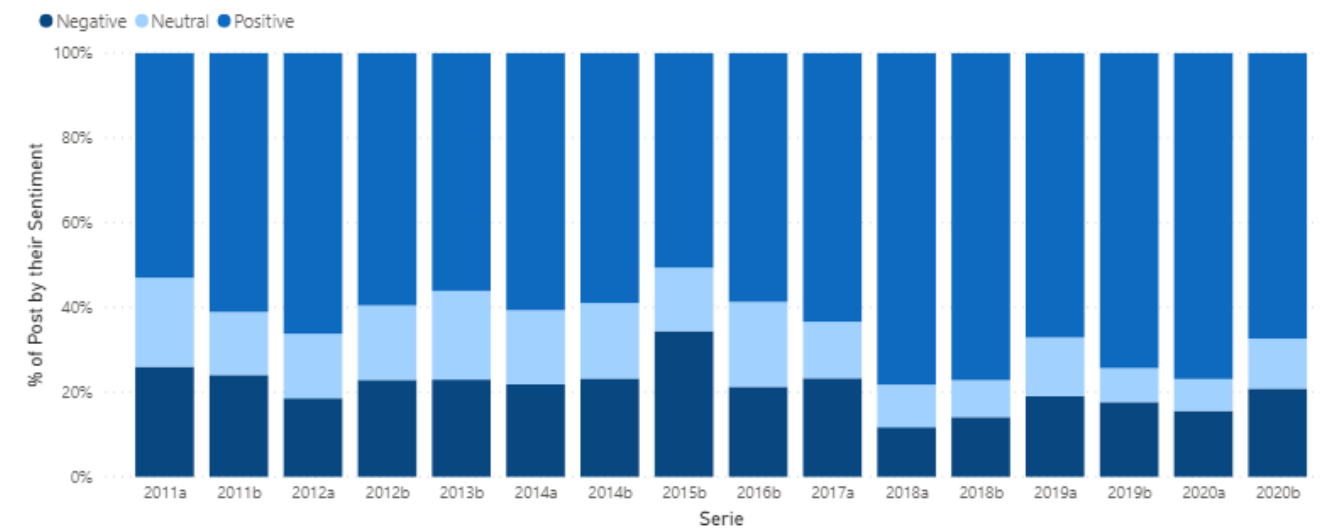

Figure 49: Distribution of positive/negative posts in Future category over the time. 


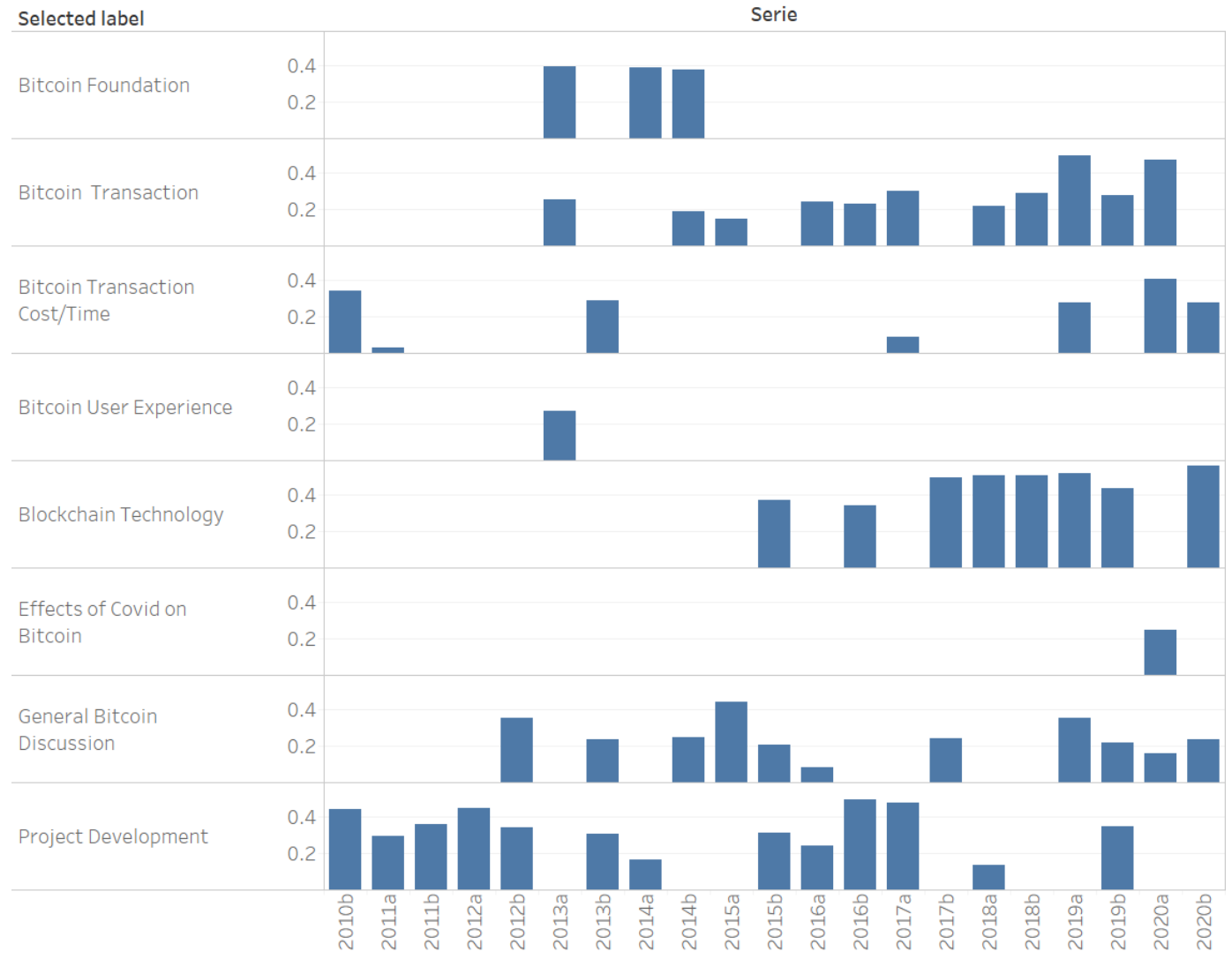

Figure 50: Compound score of topics in General category over the time.

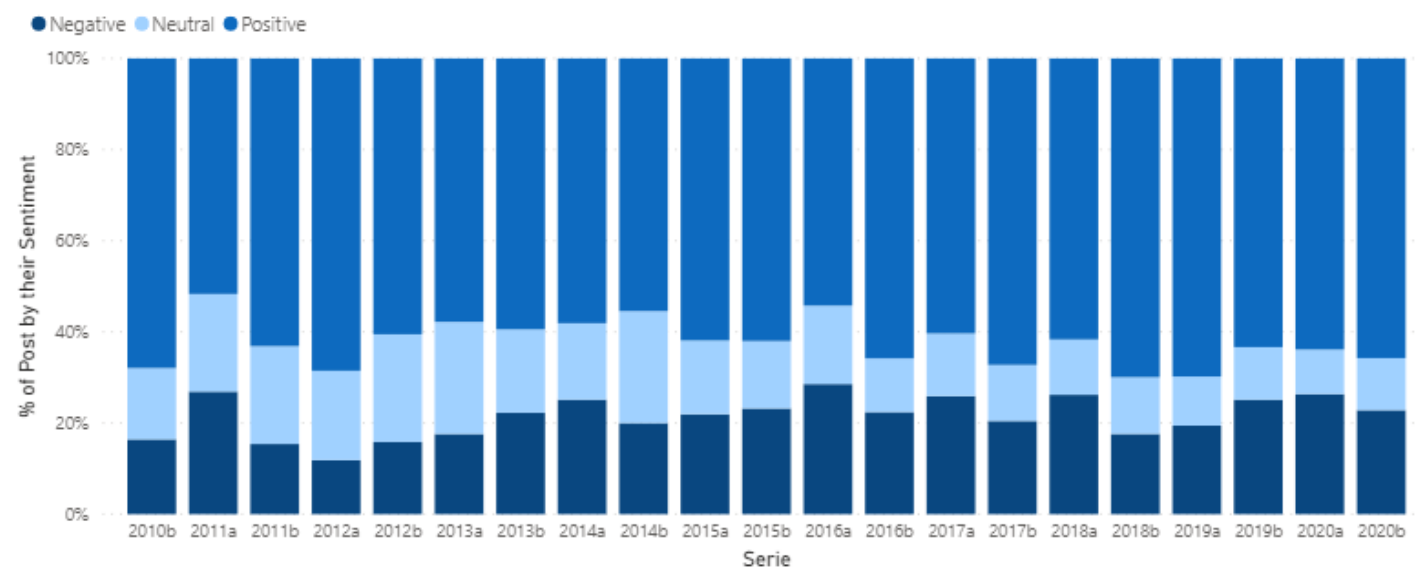

Figure 51: Distribution of positive/negative posts in General category over the time. 


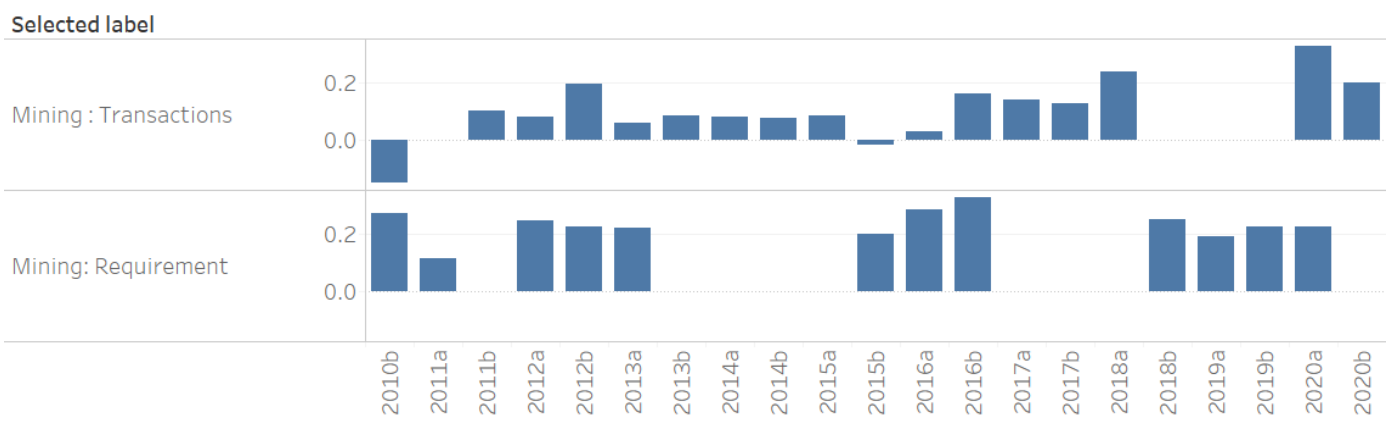

Figure 52: Compound score of topics in Mining category over the time.

\subsubsection{Mining}

In the main topic of this group which is "Mining: Transactions", we can usually see that the compound score is lower than the majority of topics of the forum and even we can find a case with the compound score of -0.15 in this topic in $2010 \mathrm{~b}$. On the other hand, in the second topic of this category which is "Mining: Requirement" we can see a monotonous sentiment weight among posts and the compound scores usually varies between 0.2 to .03. This dissimilarity can also be seen in the changing trend of positive, negative and neutral posts, and "Mining: Transactions" has fewer positive posts compared to "Mining: Requirement". The trend of changes in this category has been depicted in Figure 52 and Figure 53 .

\subsubsection{Monetary}

As we can see in Figure 54 and Figure 55, the compound scores of posts in this category are equal or slightly higher than the overall polarity on the forum. In subcategory level, Currency usually includes posts with a compound score of 0.3 and only in $2018 \mathrm{~b}$ this value has risen to 0.51 and to 0.08 in 2019a. The compound score of posts in Financial is usually between 0.2 and 0.3 and only in the last two years this value increased to 0.4 . The value of compound score is usually around 0.3 for 


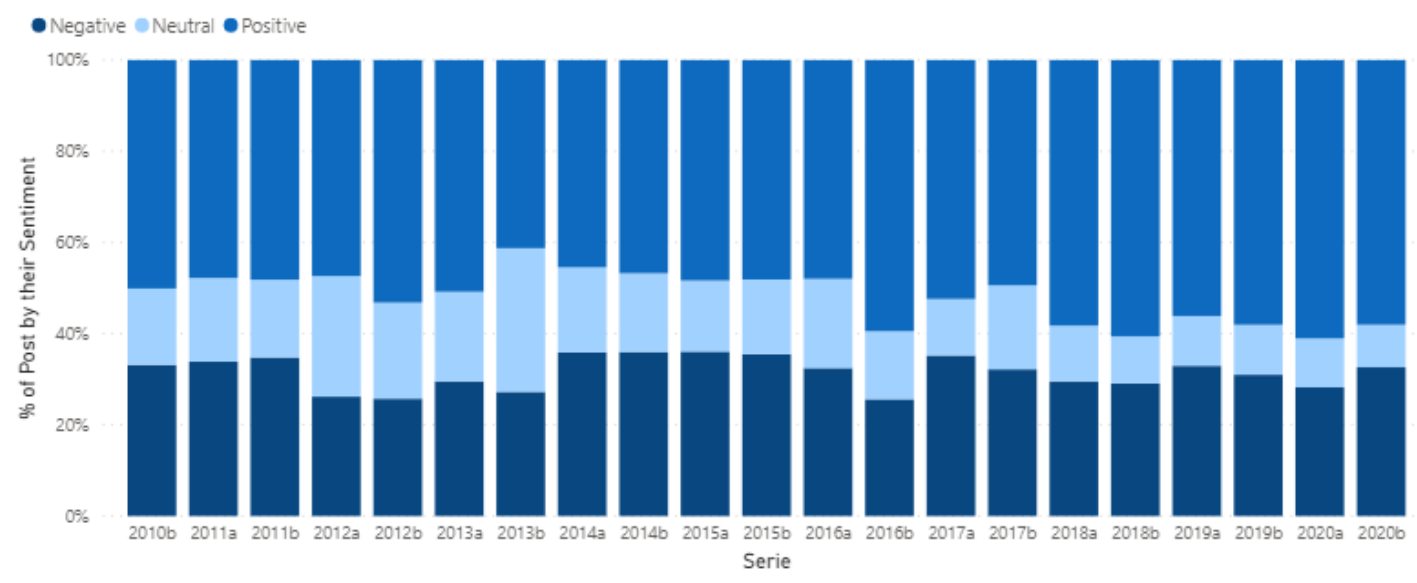

Figure 53: Distribution of positive/negative posts in Mining category over the time.

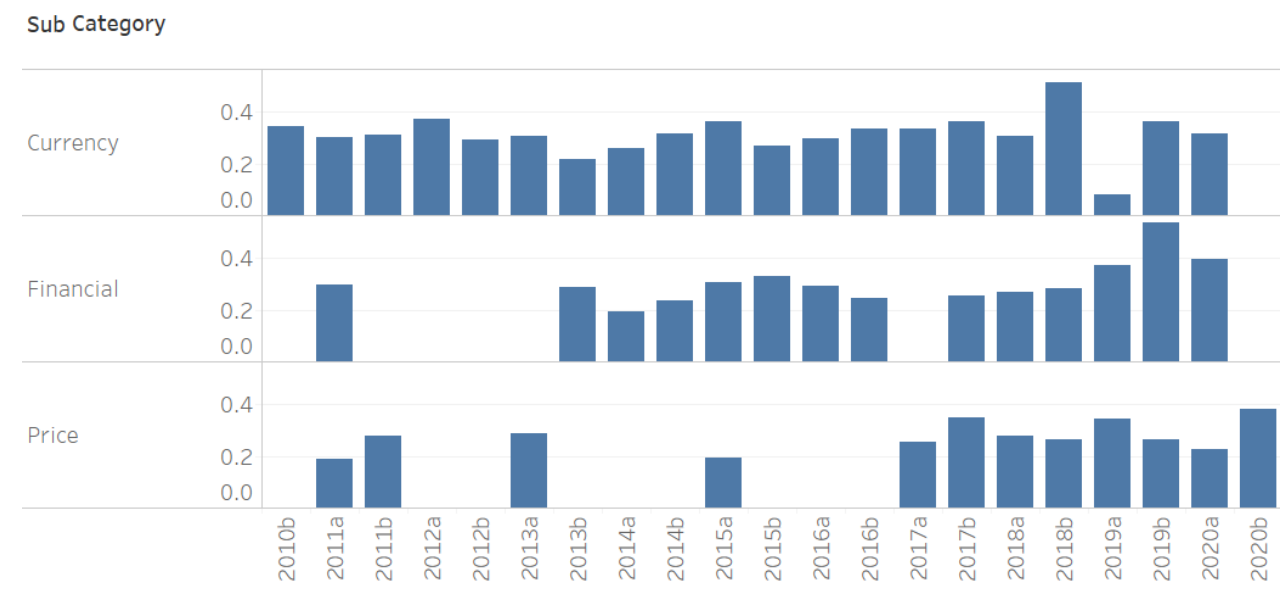

Figure 54: Compound score of subcategories in Monetary category over the time.

Price.

In topic level, we can see that "Payment with Bitcoin" usually has a positive sentiment weight and the compound score for the discussions of this topic varies between 0.29 and 0.47 (except for one case in 2013b with the value of 0.13 ) "Bitcoin Trading" which has been created in last 2 years has the compound score of higher than 0.4. The compound score of other topics could be found on Figure 56 . 


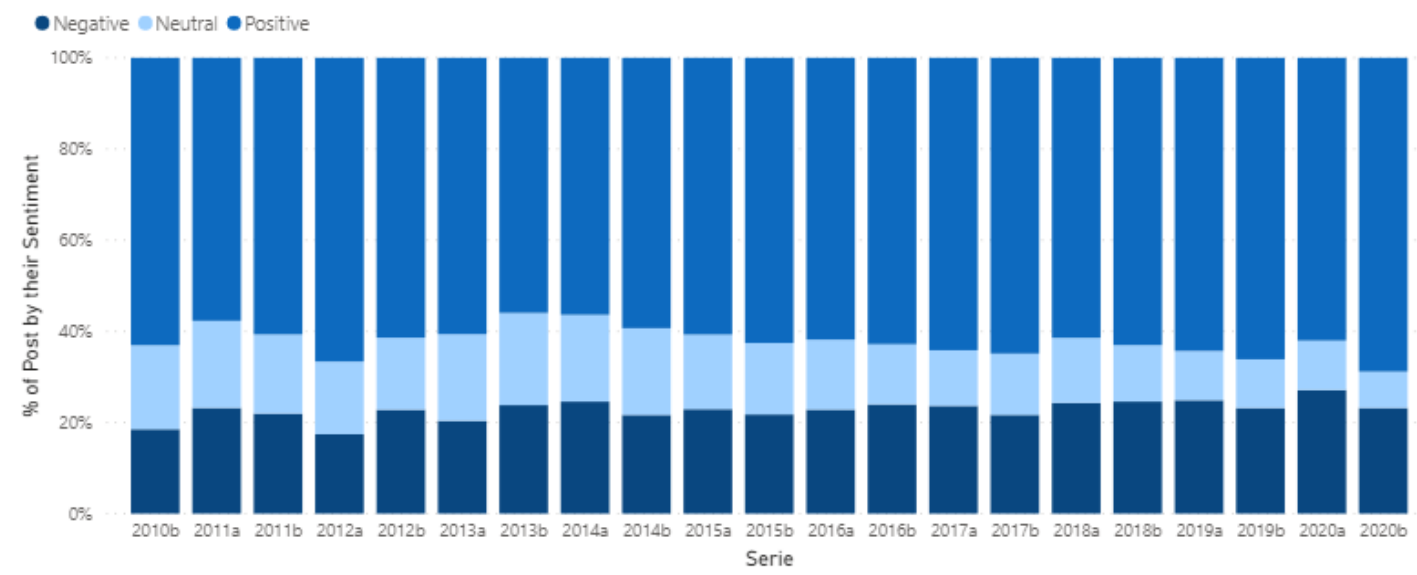

Figure 55: Distribution of positive/negative posts in Monetary category over the time.

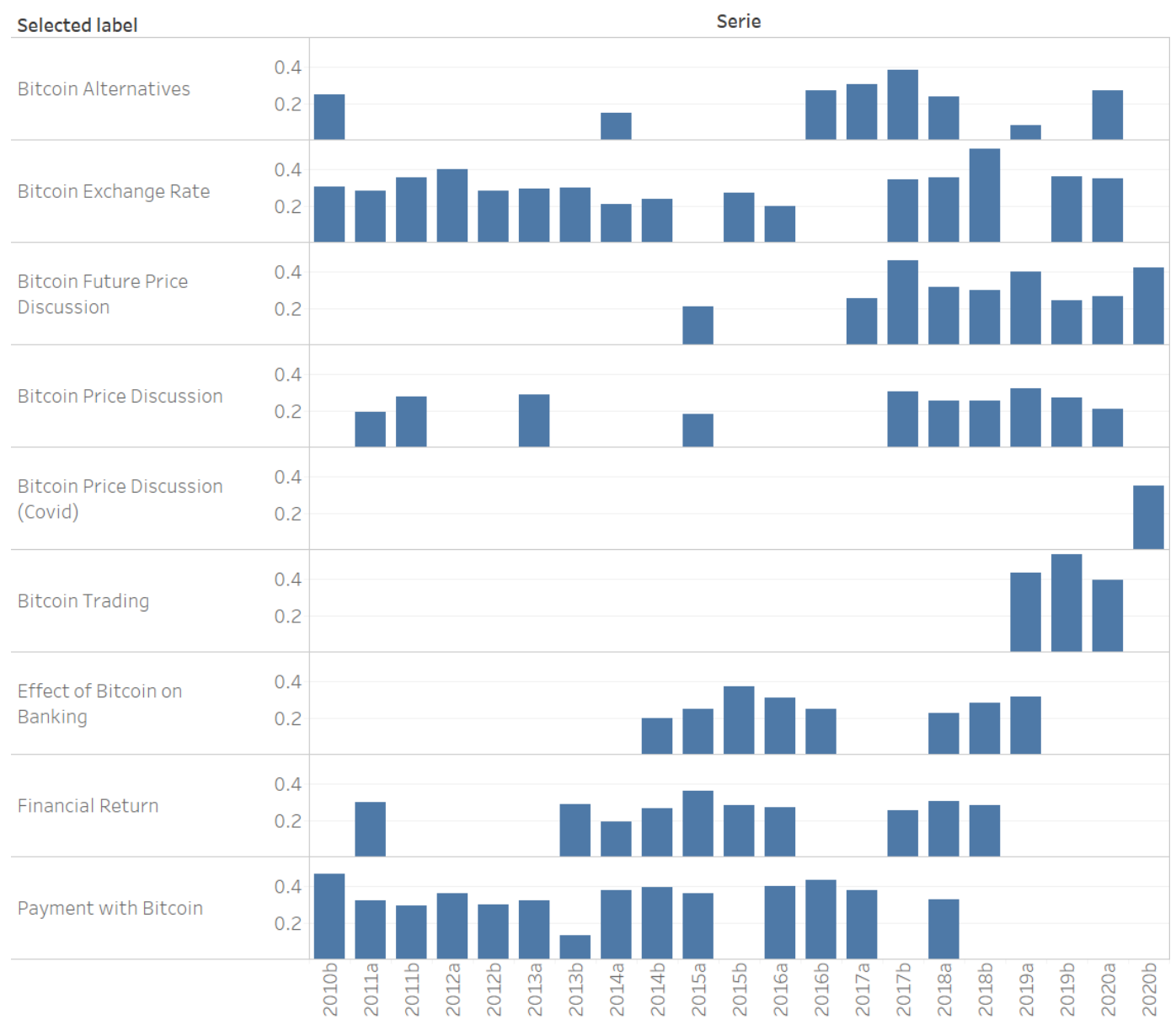

Figure 56: Compound score of topics in Monetary category over the time. 


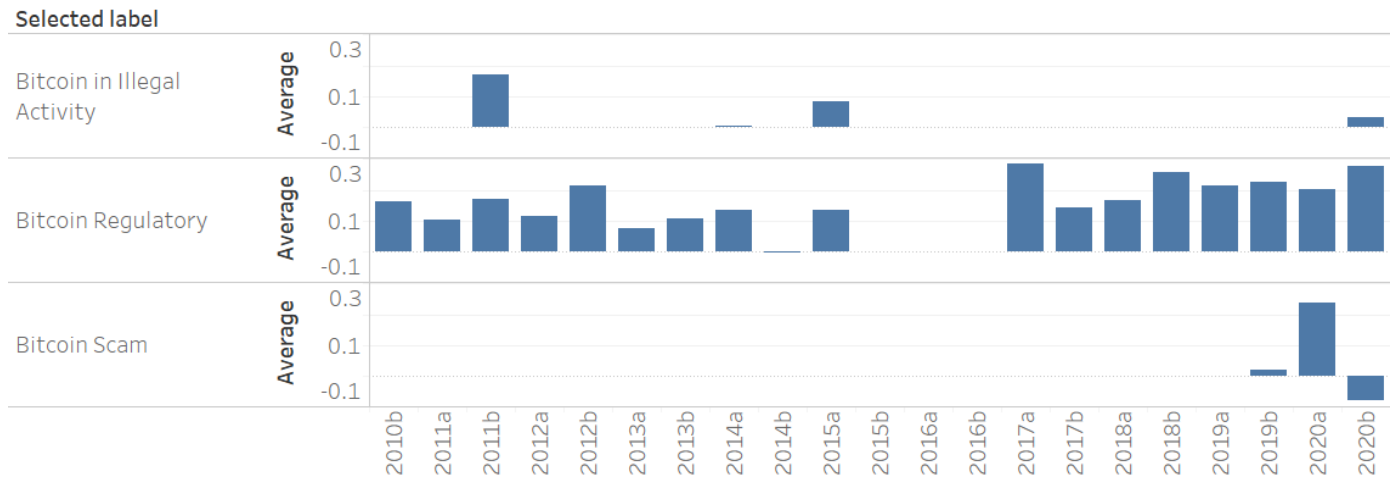

Figure 57: Compound score of topics in Regulations category over the time.

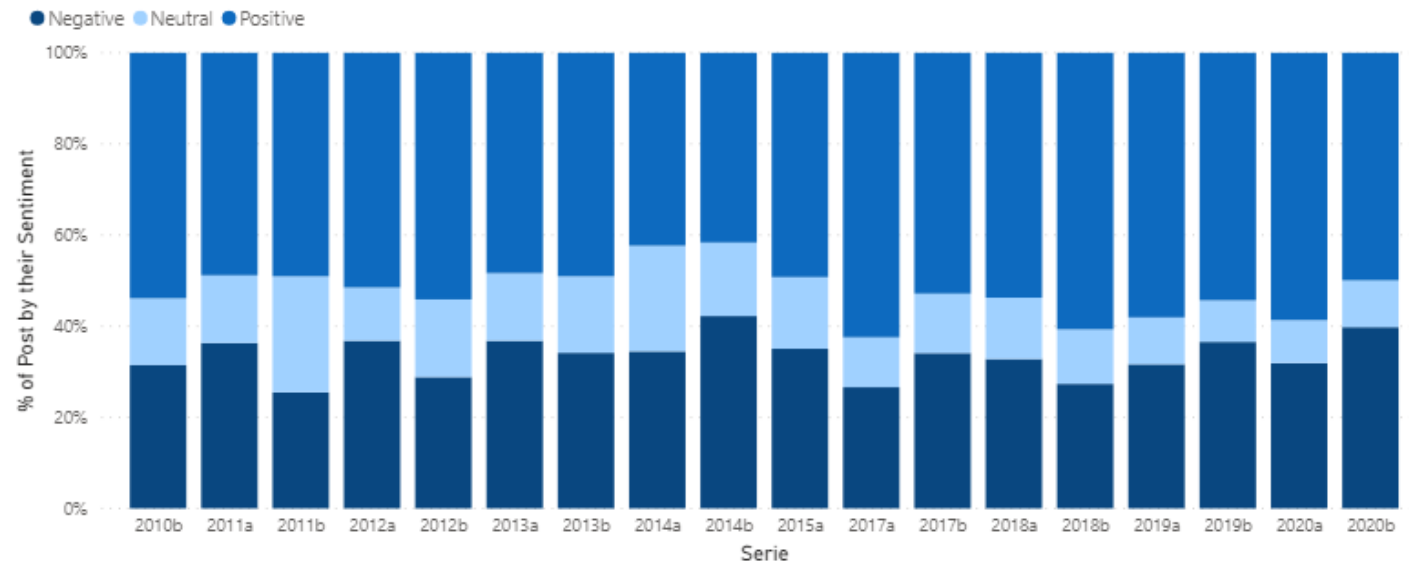

Figure 58: Distribution of positive/negative posts in Regulations category over the time.

\subsubsection{Regulations}

The topics in this category usually have low compound scores, and this value is mostly lower than the overall sentiment polarity. The compound score of posts in this topic range from 0.08 to 0.28 which indicates a tendency toward posting negative posts in this category, the numbers in most cases vary around 0.1 . The change trend of compound score of in this category can be found in Figure 57 and the distribution of positive, negative posts in Figure 58 . 


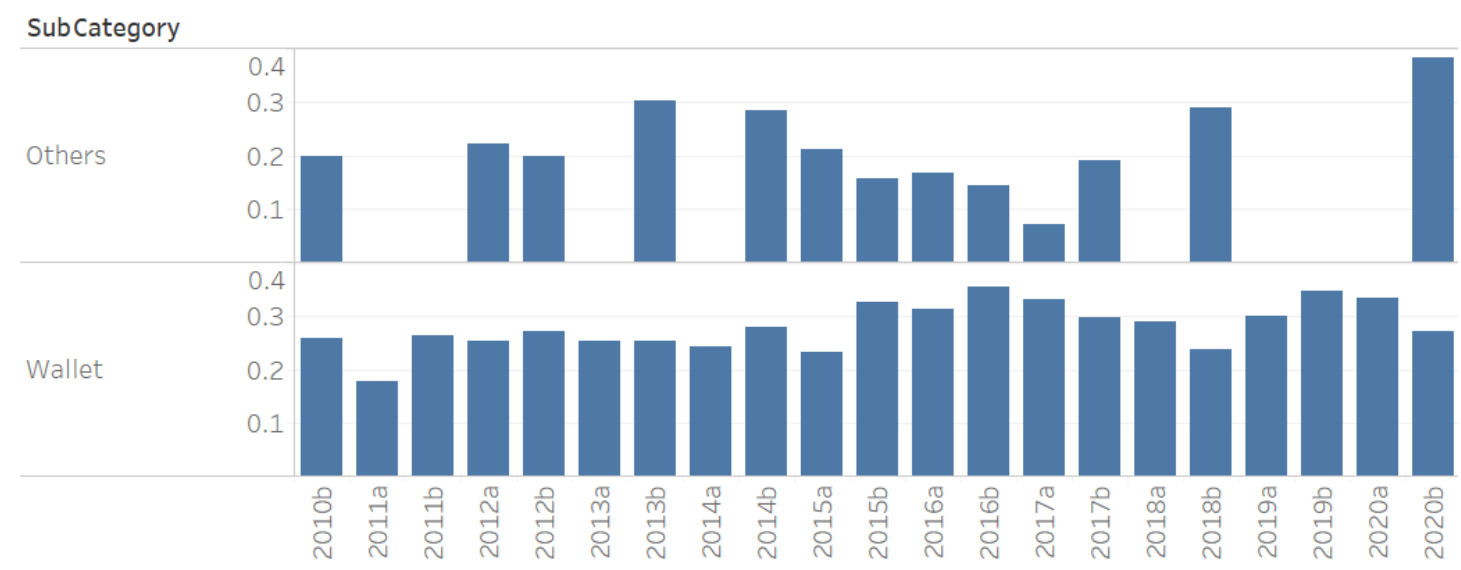

Figure 59: Compound score of subcategories in Technical category over the time.

\subsubsection{Technical}

This section has two subcategories which are Wallet and Others. As we can see in Figure 59, the compound score of posts in Wallet subcategory usually varies between 0.18 and 0.36 , and over the recent years the compound scores in this subcategory has increased. On the other hand, the range of compound scores for Others subcategory is more diverse (the numbers are in the range of 0.07 and 0.38 ) and less than the overall average.

While the range of compound scores of posts in "Fork" topic is relatively narrow ( between 0.1 and 0.4 ), these values vary between 0.1 and 0.4 in "Technical Issue" topic which is comparably a wide range. The trend of changes has been depicted in Figure 60 and Figure 61 . 


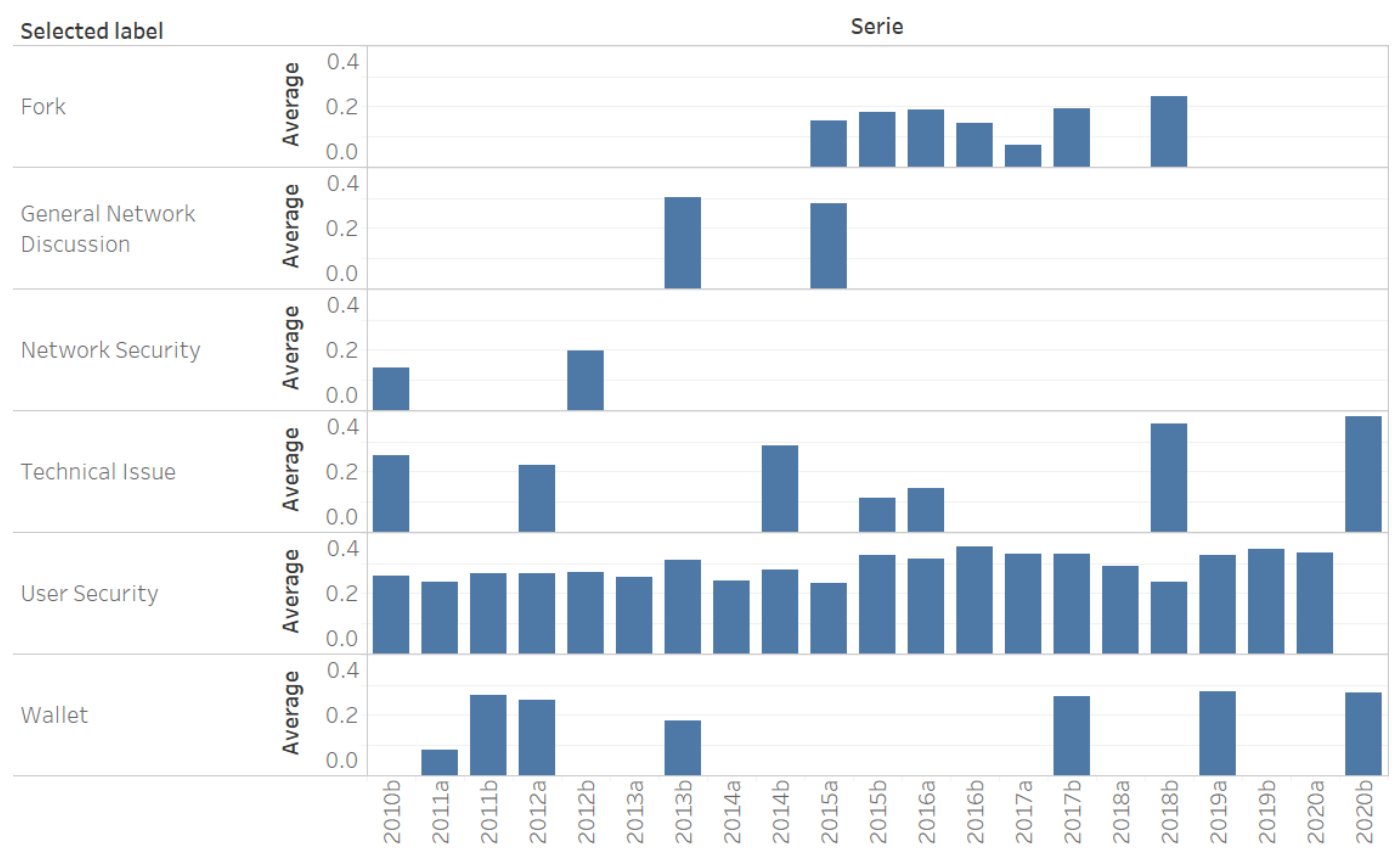

Figure 60: Compound score of topics in Technical category over the time.

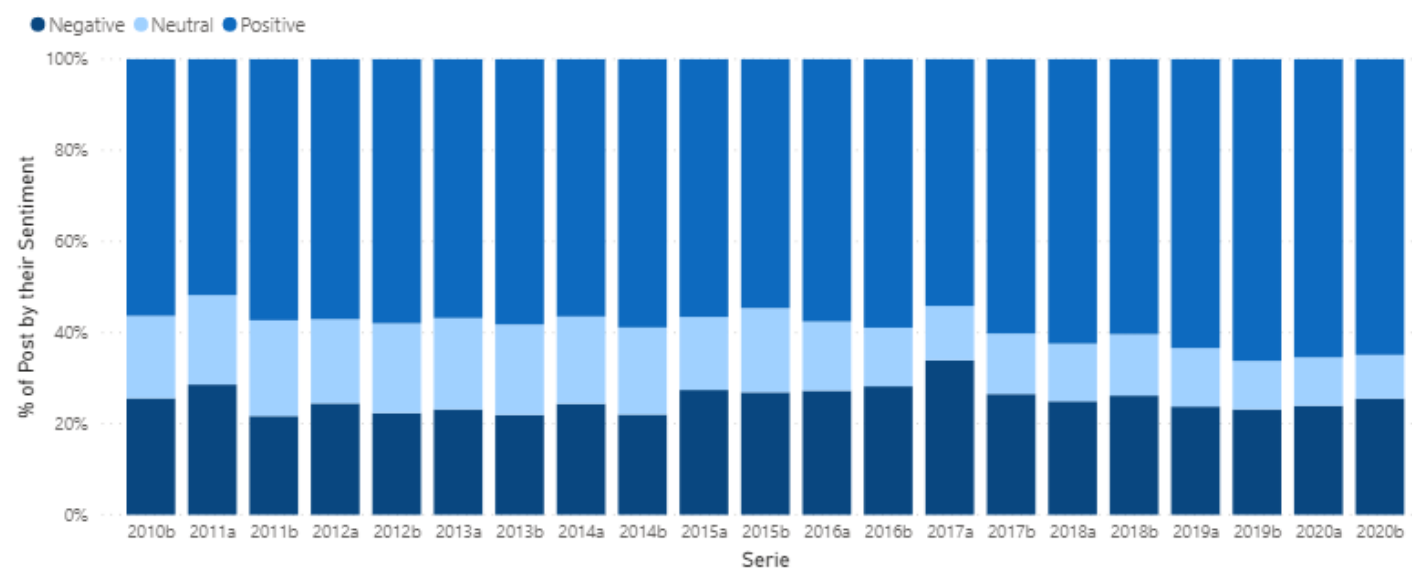

Figure 61: Distribution of positive/negative posts in Technical category over the time. 
Answer to RQ4:

Analysing the sentiment polarity of posts in the forum, we found that in general positive posts are more dominant than negative ones. Furthermore, over the recent years the percentage of neutral posts has been reduced which implies that there is a tendency towards the emergence and growth of sentiment polarity in posts. The compound score of different categories varies, and while some categories such as Monetary contain more positive topics, the average compound score of other topics such as Regulation and Mining is usually lower comparably.

\subsection{RQ5: Is there any correlation between the evo- lution of discussion topics related to Bitcoin and the change in Bitcoin prices?}

To answer this question, we evaluated the correlation of price with the parameters we had identified in previous sections. In this high level evaluation we considered two distinct prices for every period as the basis of our evaluation: 1) the average daily price over the 6 month period, and 2) the median daily price in the same period. Considering the linear nature of the correlation method and the fact that most topics were absent in one or more than one period, we evaluated the model on the category level. We implemented the model under two different circumstances: in the first state we surveyed the general relation of the number posts and the sentiment polarity with the price.

As we can see in Figure 62, the sentiment polarity of posts has a linear relation with the price of Bitcoin. But, the number of posts does not have such a linear relation with Bitcoin price. In order to make sure the Bitcoin price being independent of the number of posts, we already measured the correlation of the amount of discussions with the daily prices in the whole dataset and even under this condition, the value of 


\begin{tabular}{|c|c|c|c|c|}
\hline & $\begin{array}{l}\text { "ू } \\
\stackrel{0}{0} \\
\text { \# }\end{array}$ & 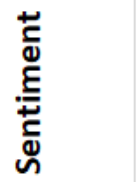 & $\stackrel{\infty}{\stackrel{一}{\varangle}}$ & 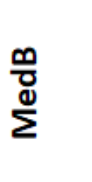 \\
\hline \# Post & 1.00 & $(0.33)$ & 0.11 & 0.04 \\
\hline Sentiment & $(0.33)$ & 1.00 & 0.61 & 0.60 \\
\hline AveB & 0.11 & 0.61 & 1.00 & 0.99 \\
\hline MedB & 0.04 & 0.60 & 0.99 & 1.00 \\
\hline
\end{tabular}

Figure 62: Overall correlation of Bitcoin price with parameters.

\begin{tabular}{|c|c|c|c|c|c|c|c|c|c|c|c|c|c|c|c|c|c|c|}
\hline & 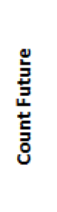 & 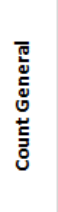 & 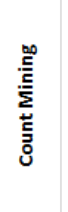 & 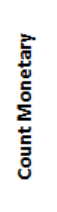 & 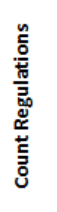 & 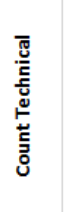 & 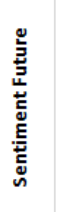 & 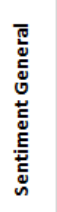 & 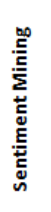 & 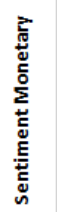 & 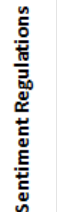 & 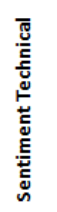 & 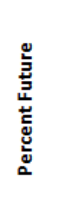 & 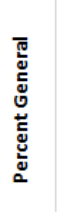 & 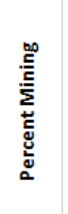 & 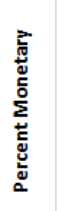 & 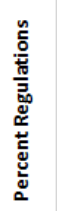 & 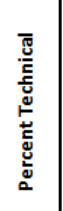 \\
\hline Ave & 0.03 & 0.24 & $(0.04)$ & 0.31 & 0.28 & $(0.09)$ & 0.77 & 0.21 & 0.65 & 0.38 & 0.06 & 0.72 & 0.27 & 0.40 & $(0.37)$ & 0.41 & 0.55 & (0.52) \\
\hline Median Price & 0.06 & 0.19 & $(0.10)$ & 0.24 & 0.23 & $(0.18)$ & 0.79 & 0.19 & 0.68 & 0.37 & 0.06 & 0.73 & 0.32 & 0.41 & $(0.36)$ & 0.37 & 0.57 & \\
\hline
\end{tabular}

Figure 63: Correlation of Bitcoin price with topic category discussion volume, sentiment and percent of discussions per topic category.

correlation was 0.19 which again confirmed our findings.

For the next stage, we evaluated the relation between Bitcoin price and the target parameters in the category level. The results of these evaluations are depicted in Figure 63 .

As we can see, the highest correlation between sentiment polarity and price is found in discussions in the Future, Technical and Mining categories . The correlation of these three categories with median price is $0.79,0.73$ and 0.68 , respectively. Furthermore, we can see that discussions about Monetary have a midmost correlation (around 0.37), and the discussions in the General category have a low correlation (around 0.20) with Bitcoin price. Regulation topic has almost no relation with the price of Bitcoin. Speaking of the number of posts, we can find that the correlation ratio of none of the categories with Bitcoin price is higher than 0.3 and the mentioned ratios can be mostly found in Monetary, Regulation and General categories which 
none of them have a tight correlation with Price. However, as we can find from the percentage of posts in this figure, there could be an intermediate correlation between the number of posts and Bitcoin price. The largest correlation ratio in this part is related to Regulation which has a correlation ratio of 0.57 compared to median price. An important point is that Technical and Mining categories have negative correlation with price $(-0.55$ and -0.36 respectively), the greater the number of posts in these categories, the lower the value of Bitcoin price.

Answer to RQ5: In this work, we found that the sentiment polarity of posts is correlated to Bitcoin price and the most correlation value for this dependency is related to discussions in Future, Technical and Mining categories, while Regulations has the least relation from this respect. On the other hand, we could not find any correlation between the number of posts and Bitcoin price. However, the amount of posts in some specific categories has a slight correlation with Bitcoin price. 


\section{Chapter 5}

\section{Discussion}

In this chapter, we present the results of our investigations. In Section 5.1, we review the results of this work and compare them with previous similar works. Then, in Section 5.2 , we discuss the implications of out work and describe threats to validity in Section 5.3 .

\section{$5.1 \quad$ Findings}

Our work was an empirical study of users' discussions in a specialized forum, and we tried to provide answers to the research questions stated in Section 1.3 by applying NLP and topic modelling techniques described in Section 3.2. We first review the results reported in Chapter 4 and next, we study and compare our findings with previous studies in this domain which we introduced in Section 2.3.2.

In this work, we study and analyzed all of the posts extracted from the bitcointalk.org forum and found 33 topics which we presented in Table 5. We then categorized the topics into six categories: Future, General, Mining, Monetary, Regulations, and Technical. In our investigations we realized that the diversity of discussions, especially in Monetary category, increased over the time as more topics could be found in this forum in comparison to earlier years. Moreover, we found 
that the discussion topics were dynamic and varying; except for the topics such as "Mining: Transaction" or "Bitcoin Regulatory", most topics appeared in the forum dynamically, thus, these changes should be monitored constantly and proper measures should be taken where these topics are used. In Section 4.2 and Section 4.3 , we analyzed the volume of posts in the forum. Our first finding was that the number of post was varying over the time and up until now; we have found three local maximums on 2011a, 2014a and 2017b which indicate the fact that in different time periods the forum had different levels of popularity among users, and experts need to consider the effect of the popularity of forum in their analysis. In our later investigation we observed that the largest growth in the number of posts happened in "Monetary" category, and the topics which were related to price were the most important topics in the forum over the past years.

According to what we found in previous papers and the importance of sentiment analysis in Bitcoin price prediction [9,64], we evaluated the sentiment polarity of the posts in Section 4.4. Generally, we can say that the overall sentiment polarity of posts in the forum is positive with the average compound score of 0.26 . Furthermore, we found that over the time the percentage of neutral posts decreased in the forum as we saw more of the posts with the positive or negative sentiment weight. In evaluation of compound score per category we found that this measure has various values in different categories. Future, General and Monetary categories with the average compound score of 0.30 have higher average compound scores. On the other hand, Technical, Mining and Regulations categories have lower compound scores. Finally, for answering the last research question we tried to measure the correlation of the number and the sentiment of posts with Bitcoin price using Pearson correlation coefficient. We did not find any relation between the number of posts and Bitcoin price. However, when considering the sentiment of posts, we found that the overall correlation of sentiment polarity of posts and Bitcoin price is more than 0.6. Moreover, 
when we evaluated this correlation in each one of the categories separately, we found that sentiment weight of posts in Future, Technical and Mining categories has relation with Bitcoin price. On the other hand, discussions in General and Regulations do not have a considerable correlation with Bitcoin price and only a slight correlation in the Monetary category.

According to these results, we evaluate our findings and compare them with previous studies from three perspectives. First, we study the way previous studies adopted topics in their works. Second, we focus on the sentiment domain and the relation between the sentiment polarity of the discussions and Bitcoin price. Finally, we talk about using Bitcointalk and other datasets in research works.

In terms of topics, among all of the papers we surveyed, Kang et al. [7], Philip et al. [8], and Lee et al. [64] adopted topic modeling in their works. Kang et al. and Lee et al. both applied topic modeling on their corpus and identified topics in the forum and adopted the results for their further steps in their work. As we found in this study, the topics in the forums are dynamic and varying, thus, it would be challenging to identify the topics accurately with a single implementation (i.e., one model) of topic modeling. Philips et al. adopted dynamic topic modeling rightly and took into account the changes of topics. In their paper, the authors only studied a single year and did not consider the matter of creation and disappearance of topics. As we found in this research, the topics are changing over the time, and the matter of the change in topics is a parameter that should be considered in building such models.

In terms of sentiment, in previous works, Mai et al. [9 and Kim et al. 64 worked on sentiment analysis domain. Kim et al. presented an overall analysis of sentiment and proposed a price predictive model using sentiment analysis. Mai et al. followed the ideas of Kang et al. [7 and categorized the forum's users and compared the difference of sentiment polarity of silent majority groups versus active 
Table 8: Datasets used in previous research.

\begin{tabular}{|l|l|l|}
\hline \multicolumn{1}{|c|}{ Paper } & \multicolumn{1}{c|}{ Time Frame } & Source \\
\hline Phillips et al. [8] & Aug 2016-Aug 2017 & reddit.com \\
\hline Kang et al. [7] & Nov 2009-Feb 2018 & bitcointalk.org \\
\hline Lee et al. 6] & Dec 2013-Sep 2016 & bitcointalk.org \\
\hline Kim et al. [64 & Dec 2013-Feb 2016 & bitcointalk.org \\
\hline Mai et al. [9] & Jan 2012-Dec 2014 & bitcointalk.org \\
\hline
\end{tabular}

users. Both works evaluated sentiment in the whole forum and did not work on the study of sentiment on topic level. On the other hand, in our study, we realized that the proportion of correlation between Bitcoin price and sentiment polarity is different for different categories. Therefore, in order to achieve more precise results, a good approach would be to work on studying the relationship between price and selected categories such as Technical and Mining.

With respect to the dataset, we present the papers that used Reddit or Bitcointalk in Table 5.1. As we can see in this table, except for [7, most of the works adopted data from a period of 1 to 4 years in their research and each one of these papers had their own justifications for choosing their datasets.

As Figure 20 in Section 4.3 suggests, the number of comments has decreased in this forum over the past two years, which is most probably the consequence of the emergence of some research and academic papers that recommend reddit.com as a forum for evaluating social media about Bitcoin [8,101]. Although we agree that currently reddit.com is a better source of data, our goal was to evaluate the change trends within a wide time span of ten years, and therefore, we decided to go with bitcointalk.org in our work. To sum up, given the fact that every forum might have different levels of popularity and different volume of discussions in different time periods, we think that the best approach for conducting a comprehensive study is 
to use a combination of different datasets to eliminate the effect of popularity or unpopularity of different sources.

\subsection{Implications}

A variety of stakeholders are involved in cryptocurrency communities such as traders, miners, users, developers and so on. We think that the findings of our research would be embraced by two categories of these stakeholders: 1) data scientists and designers of price analysis models, and 2) public beneficiaries on community and media level. We next describe the implications of our work for these two groups.

\subsubsection{Implications for Bitcoin pricing analysts}

The importance of Bitcoin price analysis has increased considerably over the past years. Only in 2020, the market value of Bitcoin has reached the price of more than 400 billion USD by the end of the year (while it used to be less than 150 billion USD in the beginning of the year). Consequently, the knowledge in this domain and the models that have the ability of predicting price have gained special attention.

First of all, the information on social networking websites are related to Bitcoin price. Similar to previous works in this domain 669,64 , we found the relation between price and discussions in the forum. In particular, we found a difference in the correlation of price with the sentiment of several categories. Therefore, sentiment analysis per category can be valuable for including in price analysis models.

Secondly, designers of the models need to pay attention to the dynamics of topics and their changes over the time. They are better to reconsider the assignments and distribution of topics time by time. Finally, speaking of data sources, we can say that generally reddit.com is a better source for real-time data. However, according to our investigations, the popularity of bitcointalk.org increased two times after 
a period of temporal unpopularity, and this might happen again in the future. As a result, we suggest researchers to use multiple resources in their work.

\subsubsection{Implications for Bitcoin community}

Bitcoin, like other cryptocurrencies, is decentralized and its governance is distributed. Therefore, the opinion of each one of the stakeholders plays some role in its popularity [3,10]. In this research, we tried to take an overall look at the topics in the forum and highlight the key trends. For instance, we found that "Bitcoin adoption" topic was created in the forum which indicates a trend for Bitcoin to become a more stable cryptocurrency from the perspective of the forum's users. As another example, we found "Bitcoin foundation" topic which is related to a period in which a NGO with the same name was established in the USA. Therefore, the results of our research provide the opportunity for the users of the forum (i.e., Bitcoin community members) to become more familiar with its potential. Additionally, several stakeholders can benefit from the results on studying the evolution of forum discussions:

- Central banks monitor different cryptocurrencies and the news related to them. We found that one of the topics of the forum is in fact "Effect of Bitcoin on Banking". Banks can monitor the expectation of the public or their response to the cryptocurrencies.

- Governments have set several rules and developed policies for managing and monitoring the financial ecosystem of Bitcoin over the past years. Review and analysis of the discussion related to regulation can provide feedback about the response of the public to such actions. Governments also can gain insights into

the use of Bitcoin in illegal activities and scams and develop new policies and legislation rules (e.g., cryptocurrency tax laws and regulations). 


\subsection{Threats to Validity}

In this research, we identified several threats to the validity of our work, and we now describe them as well as the measures we took to curb them.

- Data source: we started our work with a data source which was published by Kang et al. [7] and included information up to February 2018. This dataset contains discussions about one of the subcategories of Bitcointalk.org. According to the goal of this work of conducting a comprehensive study, we embarked on crawling and collecting the data from October 1st 2020. We thank Kang et al. authors [7] for sharing their data sources. The question is whether Bitcointalk.org has an edge over reddit.com? We discussed this matter in Section 5.1. Due to time constraints, we only used the main subject of Bitcoin Discussions, and our work does not cover all the discussion topics in this forum.

- Data preprocessing: Given the fact that preprocessing could act as a double edged sword (we might lose some part of semantic information by removing or reforming the words of the corpus), we built several models with applying different options of preprocessing algorithms to make sure we have reached an reasonable precision.

- Quality of our LDA model: The accuracy of our topic model and its imperfection in grouping related items is one limitation in this research. For tackling this one, we trained different models with different numbers of topics as mentioned in Section 3.2 .2 to make sure that we are close to the optimal distribution of contextual separation in the corpus.

- Human error in manual topic labeling: There is a possibility of human error in selecting the right label for each set. Also there is a challenge for creating a harmony in the labeling team for selecting the same name for the 
same topic in different sets. As we explained in Section 3.2.4, we made a map of topics and used it as a reference in our work. Finally, we reviewed the labels manually to check for the accuracy of labels in different sets.

- Correlation with price: In this work, we had 22 different datasets and we only evaluated the correlation with the average and median price in each one of the 6-month periods. Therefore, because of the relatively long length of time periods, the results of our work might have lower accuracy in comparison with the other studies that evaluated the daily price or over short time periods.

- Fake news/posts: In this work, we have not try to assess the quality of discussions nor identify fake users, we took the data as is and our findings may be subject to the existence of fake users who may intentionally try to manipulate other's opinion on specific topics, aka spreading fake news. 


\section{Chapter 6}

\section{Conclusions}

We conclude this thesis with the thesis statement, list of our contributions summarized in Section 6.1 and our suggestions for future directions in Section 6.2.

Thesis Statement: While discussion topics have been evolved over the past decade in bitcointalk.org, six key categories of discussions were prevalent during this time. Our findings demonstrate a relation between Bitcoin price and the sentiment polarity of the selected discussion categories on the forum.

\subsection{Summary of Contributions}

In this research we studied the evolution of Bitcoin-related topics extracted from an online forum and made the following contributions to the research community.

First, as we mentioned in Section 5.1, we performed a longitudinal study of analyzing topics extracted from the online forum for the period of 10 years; to the best of our knowledge, our work is the most comprehensive study on this topic.

Second, in our work, we extracted 31 topics related to various discussions about cryptocurrency and classified them into six categories which we labeled as Future, General, Mining, Monetary, Regulations, and Technical. 
Third, we performed an analysis of the evolution of topics in the forum and identified changes in the popularity of topics in the forum and the way some topics emerged, diminished or disappeared in discussions.

Fourth, we applied a sentiment analysis on the discussions and studied the changes of sentiment over the past 10 years and presented our findings about the difference in the sentiment polarity of different topics in this work.

Fifth, we analyzed the correlation of different factors with Bitcoin price and found the relation between Bitcoin price and the sentiment polarity for some categories, this is a novel contribution. It is worth mentioning that previous studies only worked on the correlation of sentiment and Bitcoin price on the whole data set level. But in this work we performed a fine-grained analysis by considering this correlation per discussion category and we determined that only some discussions in the forum are correlated with Bitcoin price.

Finally, our sixth contribution of this work was creating and making publicly available our comprehensive dataset about Bitcointalks discussion for any future work or replication studies.

\subsection{Future Work}

During the latest stages of this work, we identified a number of directions that could be investigated in depth in future extension of this work. In this study, we identified and labeled all the posts in the forum, identified a number of topics, assigned them to posts and performed analyses. Our findings demonstrate the correlation between the sentiment polarity of some topics and Bitcoin price, therefore, one suggestion for future direction is to build a comprehensive price analysis model using our data.

Previous studies [9] and [64 have investigated the relation between the sentiment 
polarity of posts and Bitcoin price. We took a deeper look at this subject and measured this relation between each one of the categories and cryptocurrency price. We believe that analyzing the sentiment polarity of selected categories can offer further insights and leave this potential for future researchers in this field.

In this research we identified topics using topic modeling. Topic modeling is an unsupervised learning method, and we spent a considerable time on manual labelling the topics. As a outcome of this labelling, we identified several topics, a list of relevant words and all of the posts which were relevant to each one of these topics. These labelled data could be utilized in supervised machine learning methods for classifying posts of this forum in different topics and building predictive models.

In this study, we used Bitcointalk as the main source of data. Generally, we could not find any comprehensive study that compares the results of using Bitcobintalk and Reddit as the source of information. Given the importance of this matter in predicting Bitcoin price, comparing these two forums would be next research direction. More specifically, a comprehensive and detailed study that compares different online sources of data can provide other researchers the opportunity to gain better insights into the diversity, popularity, and quality of the online social media. We hope that greater research community continues to work on this topic and advance the collective knowledge, models and techniques in this field. 


\section{List of References}

[1] D. M. Blei, "Probabilistic topic models," Communications of the ACM, vol. 55, no. 4, pp. 77-84, 2012.

[2] P. Katsiampa, S. Corbet, and B. Lucey, "Volatility spillover effects in leading cryptocurrencies: A bekk-mgarch analysis," Finance Research Letters, vol. 29, pp. 68-74, 2019.

[3] D. Tapscott and A. Tapscott, Blockchain revolution: how the technology behind bitcoin is changing money, business, and the world. Penguin, 2016.

[4] E. Bouri, D. Roubaud, and S. J. H. Shahzad, "Do bitcoin and other cryptocurrencies jump together?," The Quarterly Review of Economics and Finance, vol. 76, pp. 396-409, 2020.

[5] L. Kristoufek and M. Vosvrda, "Cryptocurrencies market efficiency ranking: Not so straightforward," Physica A: Statistical Mechanics and its Applications, vol. 531, p. 120853, 2019.

[6] Y. B. Kim, J. Lee, N. Park, J. Choo, J.-H. Kim, and C. H. Kim, "When bitcoin encounters information in an online forum: Using text mining to analyse user opinions and predict value fluctuation," PloS one, vol. 12, no. 5, p. e0177630, 2017.

[7] K. Kang, J. Choo, and Y. Kim, "Whose opinion matters? analyzing relationships between bitcoin prices and user groups in online community," Social Science Computer Review, p. 0894439319840716, 2019.

[8] R. C. Phillips and D. Gorse, "Mutual-excitation of cryptocurrency market returns and social media topics," in Proceedings of the 4 th International Conference on Frontiers of Educational Technologies, pp. 80-86, 2018. 
[9] F. Mai, Z. Shan, Q. Bai, X. Wang, and R. H. Chiang, "How does social media impact bitcoin value? a test of the silent majority hypothesis," Journal of Management Information Systems, vol. 35, no. 1, pp. 19-52, 2018.

[10] M. Crosby, P. Pattanayak, S. Verma, V. Kalyanaraman, et al., "Blockchain technology: Beyond bitcoin," Applied Innovation, vol. 2, no. 6-10, p. 71, 2016.

[11] "Some bitcoin words you might hear." https://bitcoin.org/en/vocabulary\# address Accessed: 2020-11-31).

[12] M. H. Simon London, Brant Carson, "Blockchain explained: What it is and isn't, and why it matters." https://www.mckinsey. com/business-functions/mckinsey-digital/our-insights/ blockchain-explained-what-it-is-and-isnt-and-why-it-matters (2018/09/28), Accessed: 2020-12-16.

[13] L. W. Cong and Z. He, "Blockchain disruption and smart contracts," The Review of Financial Studies, vol. 32, no. 5, pp. 1754-1797, 2019.

[14] B. Jopson, "Regulators say bitcoin poses 'financial stability risks'," Financial Times, vol. 21, 2016.

[15] "coindesk", https://www.coindesk.com/price/bitcoin Accessed: 2020-1221.

[16] H. A. Aalborg, P. Molnár, and J. E. de Vries, "What can explain the price, volatility and trading volume of bitcoin?," Finance Research Letters, vol. 29, pp. 255-265, 2019.

[17] J.-C. Gerlach, G. Demos, and D. Sornette, "Dissection of bitcoin's multiscale bubble history from january 2012 to february 2018," Royal Society open science, vol. 6, no. 7, p. 180643, 2019.

[18] L. Kristoufek, "Bitcoin meets google trends and wikipedia: Quantifying the relationship between phenomena of the internet era," Scientific reports, vol. 3, no. 1, pp. 1-7, 2013.

[19] X. Li and C. A. Wang, "The technology and economic determinants of cryptocurrency exchange rates: The case of bitcoin," Decision Support Systems, vol. 95, pp. 49-60, 2017. 
[20] S. Velankar, S. Valecha, and S. Maji, "Bitcoin price prediction using machine learning," in 2018 20th International Conference on Advanced Communication Technology (ICACT), pp. 144-147, IEEE, 2018.

[21] S. McNally, J. Roche, and S. Caton, "Predicting the price of bitcoin using machine learning," in 2018 26th Euromicro International Conference on Parallel, Distributed and Network-based Processing (PDP), pp. 339-343, IEEE, 2018.

[22] Z. H. Munim, M. H. Shakil, and I. Alon, "Next-day bitcoin price forecast," Journal of Risk and Financial Management, vol. 12, no. 2, p. 103, 2019.

[23] A. S. Hayes, "Bitcoin price and its marginal cost of production: support for a fundamental value," Applied Economics Letters, vol. 26, no. 7, pp. 554-560, 2019 .

[24] M. Campbell-Verduyn, Bitcoin and beyond. Taylor \& Francis, 2017.

[25] L. Kristoufek, "What are the main drivers of the bitcoin price? evidence from wavelet coherence analysis," PloS one, vol. 10, no. 4, p. e0123923, 2015.

[26] S. Jin, R. Ali, and A. Vlasov, "Cryptoeconomics: data application for token sales analysis," in International Conference Information Systems, 2017.

[27] S. Athey, I. Parashkevov, V. Sarukkai, and J. Xia, "Bitcoin pricing, adoption, and usage: Theory and evidence," 2016.

[28] M. Al Guindy and R. Riordan, "Tweeting the good news: Returns and price informativeness," Available at SSRN 2999443, 2017.

[29] M. Al Guindy and R. Riordan, "The social internetwork and stock returns," Available at SSRN 3501915, 2019.

[30] J. Kaminski, "Nowcasting the bitcoin market with twitter signals," arXiv preprint arXiv:1406.7577, 2014.

[31] M. Matta, I. Lunesu, and M. Marchesi, "Bitcoin spread prediction using social and web search media.," in UMAP workshops, pp. 1-10, 2015.

[32] A. M. Balfagih and V. Keselj, "Evaluating sentiment classifiers for bitcoin tweets in price prediction task," in 2019 IEEE International Conference on Big Data (Big Data), pp. 5499-5506, IEEE, 2019. 
[33] S. Corbet, B. Lucey, A. Urquhart, and L. Yarovaya, "Cryptocurrencies as a financial asset: A systematic analysis," International Review of Financial Analysis, vol. 62, pp. 182-199, 2019.

[34] H. Jelodar, Y. Wang, C. Yuan, X. Feng, X. Jiang, Y. Li, and L. Zhao, "Latent dirichlet allocation (lda) and topic modeling: models, applications, a survey," Multimedia Tools and Applications, vol. 78, no. 11, pp. 15169-15211, 2019.

[35] J. W. Mohr and P. Bogdanov, "Special issue title: Topic models and the cultural sciences," Special Issue of Poetics, vol. 41, no. 6, pp. 25-26, 2013.

[36] W. A. Qader, M. M. Ameen, and B. I. Ahmed, "An overview of bag of words; importance, implementation, applications, and challenges," in 2019 International Engineering Conference (IEC), pp. 200-204, IEEE, 2019.

[37] E. S. Kayi, K. Yadav, and H.-A. Choi, "Topic modeling based classification of clinical reports," in 51st Annual Meeting of the Association for Computational Linguistics Proceedings of the Student Research Workshop, pp. 67-73, 2013.

[38] R. Zhao and K. Mao, "Fuzzy bag-of-words model for document representation," IEEE transactions on fuzzy systems, vol. 26, no. 2, pp. 794-804, 2017.

[39] S. Deerwester, S. T. Dumais, G. W. Furnas, T. K. Landauer, and R. Harshman, "Indexing by latent semantic analysis," Journal of the American society for information science, vol. 41, no. 6, pp. 391-407, 1990.

[40] D. M. Blei, A. Y. Ng, and M. I. Jordan, "Latent dirichlet allocation," Journal of machine Learning research, vol. 3, no. Jan, pp. 993-1022, 2003.

[41] D. M. Blei, A. Y. Ng, and M. I. Jordan, "Latent dirichlet allocation," in $A d-$ vances in neural information processing systems, pp. 601-608, 2002.

[42] D. Blei, A. Ng, and M. Jordan, "Latent dirichlet allocation," Advances in neural information processing systems, vol. 14, pp. 601-608, 2001.

[43] C.-K. Yau, A. Porter, N. Newman, and A. Suominen, "Clustering scientific documents with topic modeling," Scientometrics, vol. 100, no. 3, pp. 767-786, 2014.

[44] A. Suominen and H. Toivanen, "Map of science with topic modeling: Comparison of unsupervised learning and human-assigned subject classification," Journal of the Association for Information Science and Technology, vol. 67, no. 10, pp. 2464-2476, 2016. 
[45] B. Carron-Arthur, J. Reynolds, K. Bennett, A. Bennett, and K. M. Griffiths, "What's all the talk about? topic modelling in a mental health internet support group," BMC psychiatry, vol. 16, no. 1, p. 367, 2016.

[46] A. Abdellatif, D. Costa, K. Badran, R. Abdalkareem, and E. Shihab, "Challenges in chatbot development: A study of stack overflow posts," in Proceedings of the 17th International Conference on Mining Software Repositories, pp. 174$185,2020$.

[47] F. Martin and M. Johnson, "More efficient topic modelling through a noun only approach," in Proceedings of the Australasian Language Technology Association Workshop 2015, pp. 111-115, 2015.

[48] J. Chang, J. Boyd-Graber, C. Wang, S. Gerrish, and D. M. Blei, "Reading tea leaves: How humans interpret topic models," in Neural Information Processing Systems, 2009.

[49] A. Agrawal, W. Fu, and T. Menzies, "What is wrong with topic modeling?(and how to fix it using search-based se)," arXiv preprint arXiv:1608.08176, 2016.

[50] N. Székely and J. vom Brocke, "What can we learn from corporate sustainability reporting? deriving propositions for research and practice from over 9,500 corporate sustainability reports published between 1999 and 2015 using topic modelling technique," PloS one, vol. 12, no. 4, p. e0174807, 2017.

[51] J. Savoy, "Authorship attribution based on a probabilistic topic model," Information Processing \& Management, vol. 49, no. 1, pp. 341-354, 2013.

[52] B. Zheng, D. C. McLean, and X. Lu, "Identifying biological concepts from a protein-related corpus with a probabilistic topic model," BMC bioinformatics, vol. 7, no. 1, p. 58, 2006.

[53] D. J. Carter, J. Brown, and A. Rahmani, "Reading the high court at a distance: Topic modelling the legal subject matter and judicial activity of the high court of australia, 1903-2015," UNSWLJ, vol. 39, p. 1300, 2016.

[54] D. Mimno, "Computational historiography: Data mining in a century of classics journals," Journal on Computing and Cultural Heritage (JOCCH), vol. 5, no. 1, pp. 1-19, 2012.

[55] T. Kohonen, Self-organizing maps, vol. 30. Springer Science \& Business Media, 2012. 
[56] "Kohonen self-organizing maps." , https://towardsdatascience.com/ kohonen-self-organizing-maps-a29040d688da Accessed: 2021-01-25.

[57] R. Rzeszutek, D. Androutsos, and M. Kyan, "Self-organizing maps for topic trend discovery," IEEE Signal Processing Letters, vol. 17, no. 6, pp. 607-610, 2010.

[58] T.-H. Chen, S. W. Thomas, and A. E. Hassan, "A survey on the use of topic models when mining software repositories," Empirical Software Engineering, vol. 21, no. 5, pp. 1843-1919, 2016.

[59] R. Arora and B. Ravindran, "Latent dirichlet allocation based multi-document summarization," in Proceedings of the second workshop on Analytics for noisy unstructured text data, pp. 91-97, 2008.

[60] Z. Zhai, B. Liu, H. Xu, and P. Jia, "Constrained lda for grouping product features in opinion mining," in Pacific-Asia Conference on Knowledge Discovery and Data Mining, pp. 448-459, Springer, 2011.

[61] D. Nolasco and J. Oliveira, "Subevents detection through topic modeling in social media posts," Future Generation Computer Systems, vol. 93, pp. 290303, 2019.

[62] D. Naskar, S. Mokaddem, M. Rebollo, and E. Onaindia, "Sentiment analysis in social networks through topic modeling," in Proceedings of the Tenth International Conference on Language Resources and Evaluation (LREC'16), pp. 46-53, 2016.

[63] J. He, L. Li, Y. Wang, and X. Wu, "Targeted aspects oriented topic modeling for short texts," Applied Intelligence, pp. 1-16, 2020.

[64] Y. B. Kim, J. G. Kim, W. Kim, J. H. Im, T. H. Kim, S. J. Kang, and C. H. Kim, "Predicting fluctuations in cryptocurrency transactions based on user comments and replies," PloS one, vol. 11, no. 8, p. e0161197, 2016.

[65] F. Mai, Q. Bai, J. Shan, X. S. Wang, and R. H. Chiang, "The impacts of social media on bitcoin performance," 2015.

[66] R. C. Phillips and D. Gorse, "Predicting cryptocurrency price bubbles using social media data and epidemic modelling," in 2017 IEEE Symposium Series on Computational Intelligence (SSCI), pp. 1-7, IEEE, 2017. 
[67] C. Gilbert and E. Hutto, "Vader: A parsimonious rule-based model for sentiment analysis of social media text," in Eighth International Conference on Weblogs and Social Media (ICWSM-14). Available at (20/04/16) http://comp. social. gatech. edu/papers/icwsm14. vader. hutto. pdf, vol. 81, p. 82, 2014.

[68] C. W. Granger, B.-N. Huangb, and C.-W. Yang, "A bivariate causality between stock prices and exchange rates: evidence from recent asianflu," The Quarterly Review of Economics and Finance, vol. 40, no. 3, pp. 337-354, 2000.

[69] R. Koch, The 80/20 Principle: The Secret of Achieving More with Less: Updated 20th anniversary edition of the productivity and business classic. Hachette UK, 2011.

[70] T. Islam, "Ex-twit: Explainable twitter mining on health data," arXiv preprint arXiv:1906.02132, 2019.

[71] J. Abel and W. Teahan, "Universal text preprocessing for data compression," IEEE Transactions on Computers, vol. 54, no. 5, pp. 497-507, 2005.

[72] B. Srinivasa-Desikan, Natural Language Processing and Computational Linguistics: A practical guide to text analysis with Python, Gensim, spaCy, and Keras. Packt Publishing Ltd, 2018.

[73] D. O'callaghan, D. Greene, J. Carthy, and P. Cunningham, "An analysis of the coherence of descriptors in topic modeling," Expert Systems with Applications, vol. 42, no. 13, pp. 5645-5657, 2015.

[74] M. Röder, A. Both, and A. Hinneburg, "Exploring the space of topic coherence measures," in Proceedings of the eighth ACM international conference on Web search and data mining, pp. 399-408, 2015.

[75] S. Syed and M. Spruit, "Full-text or abstract? examining topic coherence scores using latent dirichlet allocation," in 2017 IEEE International conference on data science and advanced analytics (DSAA), pp. 165-174, IEEE, 2017.

[76] H. Bai, Z. Chen, M. R. Lyu, I. King, and Z. Xu, "Neural relational topic models for scientific article analysis," in Proceedings of the 27th ACM International Conference on Information and Knowledge Management, pp. 27-36, 2018.

[77] D. Greene and J. P. Cross, "Exploring the political agenda of the european parliament using a dynamic topic modeling approach," Political Analysis, vol. 25, no. 1, pp. 77-94, 2017. 
[78] N. Novielli, D. Girardi, and F. Lanubile, "A benchmark study on sentiment analysis for software engineering research," in 2018 IEEE/ACM 15th International Conference on Mining Software Repositories (MSR), pp. 364-375, IEEE, 2018.

[79] S. Elbagir and J. Yang, "Twitter sentiment analysis using natural language toolkit and vader sentiment," in Proceedings of the International MultiConference of Engineers and Computer Scientists, pp. 122-16, 2019.

[80] J. P. Pinto and V. Murari, "Real time sentiment analysis of political twitter data using machine learning approach," International Research Journal of Engineering and Technology (IRJET), vol. 6, no. 4, 2019.

[81] M. Sullivan and J. Verhoosel, Statistics: Informed decisions using data. Pearson Boston, MA, 2013.

[82] "Jupiter Notebook", https://jupyter.org/ Accessed: 2020-12-16.

[83] "Visual Studio Code", https://code.visualstudio.com/docs/setup/linux Accessed: 2020-12-16.

[84] https://docs.python.org/3/library/re.html Accessed: 2020-12-16.

[85] "nltk", https://www.nltk.org/ Accessed: 2020-12-16.

[86] "spaCy", https://spacy.io/ Accessed: 2020-12-16.

[87] "gensim", https://pypi.org/project/gensim/ Accessed: 2020-12-16.

[88] "Mallet LDA", http://mallet.cs.umass.edu/ Accessed: 2020-12-16.

[89] "pyLDAvis", https://pypi.org/project/pyLDAvis/ Accessed: 2020-12-16.

[90] "Numpy", https://numpy.org Accessed: 2020-12-16.

[91] "Pandas", https://pandas.pydata.org/ Accessed: 2020-12-16.

[92] "Vader Sentiment", https://pypi.org/project/vaderSentiment/Accessed: 2020-12-16.

[93] "PowerBI", https://powerbi.microsoft.com/en-us/ Accessed: 2020-12-16.

[94] "Tableau", https://www.tableau.com/ Accessed: 2020-12-16.

[95] "r-project", https://www.r-project.org/about.html Accessed: 2020-12-16. 
[96] "ggplot2 library in R", https://ggplot2.tidyverse.org/Accessed: 2020-1216.

[97] "ggthemes library in R", https://cran.r-project.org/web/packages/ ggthemes/index.html Accessed: 2020-12-16.

[98] "dplyr library in R", https://www.rdocumentation.org/packages/dplyr/ versions/0.7.8 Accessed: 2020-12-16.

[99] "corrgram library in R", https://cran.r-project.org/web/packages/ corrgram/index.html Accessed: 2020-12-16.

[100] "corrplot library in R", https://cran.r-project.org/web/packages/ corrplot/vignettes/corrplot-intro.html Accessed: 2020-12-16.

[101] A. Burnie and E. Yilmaz, "An analysis of the change in discussions on social media with bitcoin price," in Proceedings of the 42nd International ACM SIGIR Conference on Research and Development in Information Retrieval, pp. 889892, 2019. 
Appendix A

Sentiment Polarity Analysis Results 
Table 9: Distribution of positive/negative posts in categories/subcategories over the time.

\begin{tabular}{|c|c|c|c|c|c|c|c|c|c|c|c|c|c|c|c|c|c|c|c|c|c|c|}
\hline Category/Topic & $2010 \mathrm{~b}$ & 2011a & $2011 \mathrm{~b}$ & $2012 \mathrm{a}$ & $2012 \mathrm{~b}$ & 2013a & $2013 \mathrm{~b}$ & 2014a & $2014 \mathrm{~b}$ & $2015 \mathrm{a}$ & $2015 \mathrm{~b}$ & $2016 \mathrm{a}$ & $2016 \mathrm{~b}$ & $2017 \mathrm{a}$ & $2017 \mathrm{~b}$ & 2018a & $2018 \mathrm{~b}$ & 2019a & $2019 \mathrm{~b}$ & 2020a & $2020 \mathrm{~b}$ & Total \\
\hline Future & & 3361 & 1218 & 933 & \begin{tabular}{|l|l|}
1350 \\
\end{tabular} & & \begin{tabular}{|l|l|}
1180 \\
\end{tabular} & 2218 & 1489 & & 2256 & & 1143 & 2014 & & 2500 & 1582 & 970 & 889 & \begin{tabular}{|l|l|}
879 \\
\end{tabular} & 1343 & 25325 \\
\hline Negative & & $26 \%$ & $24 \%$ & \begin{tabular}{|l|}
$19 \%$ \\
\end{tabular} & $23 \%$ & & $23 \%$ & $22 \%$ & $23 \%$ & & $34 \%$ & & $21 \%$ & $23 \%$ & & $12 \%$ & $14 \%$ & $19 \%$ & $18 \%$ & $16 \%$ & $21 \%$ & $22 \%$ \\
\hline Neutral & & $21 \%$ & $15 \%$ & $15 \%$ & $18 \%$ & & $21 \%$ & $17 \%$ & $18 \%$ & & $15 \%$ & & $20 \%$ & $13 \%$ & & $10 \%$ & $9 \%$ & $14 \%$ & $8 \%$ & $8 \%$ & $12 \%$ & $15 \%$ \\
\hline Positive & & $53 \%$ & $61 \%$ & $66 \%$ & $60 \%$ & & $56 \%$ & $61 \%$ & $59 \%$ & & $51 \%$ & & $59 \%$ & $63 \%$ & & $78 \%$ & $77 \%$ & $67 \%$ & $74 \%$ & $77 \%$ & $67 \%$ & $63 \%$ \\
\hline General & 627 & 4205 & 2417 & 1290 & 2191 & 4501 & \begin{tabular}{|l|l|}
4448 \\
\end{tabular} & 5314 & 6496 & 3563 & 5454 & 4147 & 3503 & 7240 & 6343 & 10235 & 2715 & 4777 & 4520 & 4171 & 1673 & 89830 \\
\hline Negative & $16 \%$ & $27 \%$ & $15 \%$ & $12 \%$ & $\begin{array}{l}16 \% \\
\end{array}$ & $18 \%$ & $22 \%$ & $25 \%$ & $20 \%$ & $22 \%$ & $23 \%$ & $29 \%$ & $22 \%$ & $26 \%$ & $20 \%$ & $26 \%$ & $\begin{array}{l}18 \% \\
\end{array}$ & \begin{tabular}{|l|l|}
$19 \%$ \\
\end{tabular} & $25 \%$ & $26 \%$ & $23 \%$ & $23 \%$ \\
\hline Neutral & $16 \%$ & $21 \%$ & $21 \%$ & $20 \%$ & $24 \%$ & $25 \%$ & $18 \%$ & $17 \%$ & $25 \%$ & $16 \%$ & $15 \%$ & $17 \%$ & $12 \%$ & $14 \%$ & $12 \%$ & $12 \%$ & $12 \%$ & $11 \%$ & $12 \%$ & $10 \%$ & $11 \%$ & $16 \%$ \\
\hline Positive & $68 \%$ & $52 \%$ & $63 \%$ & $\begin{array}{ll}69 \% \\
\end{array}$ & \begin{tabular}{|l|l|}
$61 \%$ \\
\end{tabular} & $58 \%$ & $59 \%$ & \begin{tabular}{|l|l|}
$58 \%$ \\
\end{tabular} & $55 \%$ & $\begin{array}{l}62 \% \\
\end{array}$ & $\begin{array}{l}62 \% \\
\end{array}$ & $54 \%$ & $66 \%$ & $60 \%$ & \begin{tabular}{|l|}
$67 \%$ \\
\end{tabular} & $\begin{array}{ll}62 \% \\
\end{array}$ & $70 \%$ & $70 \%$ & $63 \%$ & \begin{tabular}{|l|l|}
$64 \%$ \\
\end{tabular} & $\begin{array}{l}66 \% \\
\end{array}$ & $\begin{array}{ll}61 \% \\
\end{array}$ \\
\hline Mining & \begin{tabular}{|l|}
1010 \\
\end{tabular} & 2088 & 1837 & 2245 & 1476 & 4305 & 2492 & 2987 & 1861 & 2159 & 4643 & \begin{tabular}{|l|}
3791 \\
\end{tabular} & 2470 & 1561 & 6967 & 4633 & 1492 & 1053 & 1294 & \begin{tabular}{|l|l|}
1807 \\
\end{tabular} & 567 & 52738 \\
\hline Negative & $33 \%$ & $34 \%$ & $35 \%$ & $26 \%$ & $26 \%$ & $30 \%$ & $27 \%$ & $36 \%$ & $36 \%$ & $36 \%$ & $36 \%$ & $32 \%$ & $26 \%$ & $35 \%$ & $32 \%$ & $30 \%$ & $29 \%$ & $33 \%$ & $31 \%$ & $28 \%$ & $33 \%$ & $32 \%$ \\
\hline Neutral & $17 \%$ & $18 \%$ & $17 \%$ & $26 \%$ & $21 \%$ & $20 \%$ & $32 \%$ & $19 \%$ & $17 \%$ & $16 \%$ & $16 \%$ & $20 \%$ & $15 \%$ & $12 \%$ & $18 \%$ & $12 \%$ & $10 \%$ & $11 \%$ & $11 \%$ & $11 \%$ & $9 \%$ & $17 \%$ \\
\hline Positive & $50 \%$ & \begin{tabular}{|l|l|}
$48 \%$ \\
\end{tabular} & $48 \%$ & \begin{tabular}{|l|l|}
$47 \%$ \\
\end{tabular} & \begin{tabular}{|l|}
$53 \%$ \\
\end{tabular} & $51 \%$ & \begin{tabular}{|l|l|}
$41 \%$ \\
\end{tabular} & $\begin{array}{l}45 \% \\
\end{array}$ & \begin{tabular}{|l|l|}
$47 \%$ \\
\end{tabular} & \begin{tabular}{|l|}
$48 \%$ \\
\end{tabular} & $\begin{array}{l}48 \% \\
\end{array}$ & $48 \%$ & \begin{tabular}{|l|}
$59 \%$ \\
\end{tabular} & $52 \%$ & \begin{tabular}{|l|l|}
$49 \%$ \\
\end{tabular} & $58 \%$ & \begin{tabular}{|l|l|}
$61 \%$ \\
\end{tabular} & $56 \%$ & $58 \%$ & \begin{tabular}{|l|l|}
$61 \%$ \\
\end{tabular} & $\begin{array}{l}58 \% \\
\end{array}$ & $51 \%$ \\
\hline Monetary & 1107 & \begin{tabular}{|l|l|}
7400 \\
\end{tabular} & 6992 & 2744 & 2306 & \begin{tabular}{|l|l|}
4942 \\
\end{tabular} & 4726 & 11166 & 8765 & \begin{tabular}{|l|}
10961 \\
\end{tabular} & 5210 & 8161 & 4635 & 6771 & 23635 & \begin{tabular}{|l|}
25193 \\
\end{tabular} & 9912 & 6143 & 5336 & 5040 & 1190 & 162335 \\
\hline Negative & $19 \%$ & $23 \%$ & $22 \%$ & $17 \%$ & $23 \%$ & $20 \%$ & $24 \%$ & $25 \%$ & $22 \%$ & $23 \%$ & $22 \%$ & $23 \%$ & $24 \%$ & $24 \%$ & $22 \%$ & $24 \%$ & $25 \%$ & $25 \%$ & $23 \%$ & $27 \%$ & $23 \%$ & $23 \%$ \\
\hline Neutral & $18 \%$ & $\begin{array}{l}19 \% \\
\end{array}$ & $17 \%$ & $16 \%$ & $16 \%$ & $19 \%$ & $20 \%$ & $19 \%$ & \begin{tabular}{|l|}
$19 \%$ \\
\end{tabular} & $16 \%$ & $16 \%$ & $15 \%$ & $13 \%$ & $12 \%$ & $13 \%$ & $14 \%$ & $12 \%$ & $11 \%$ & $11 \%$ & $11 \%$ & $8 \%$ & $15 \%$ \\
\hline Positive & $63 \%$ & \begin{tabular}{|l|l|}
$58 \%$ \\
\end{tabular} & $61 \%$ & $\begin{array}{l}67 \% \\
\end{array}$ & \begin{tabular}{|l|l|}
$61 \%$ \\
\end{tabular} & \begin{tabular}{|l|l|}
$61 \%$ \\
\end{tabular} & $56 \%$ & $56 \%$ & \begin{tabular}{|l|l|}
$59 \%$ \\
\end{tabular} & \begin{tabular}{|l}
$61 \%$ \\
\end{tabular} & $\begin{array}{l}63 \% \\
\end{array}$ & $\begin{array}{ll}62 \% \\
\end{array}$ & $\begin{array}{l}63 \% \\
\end{array}$ & \begin{tabular}{|l|l|}
$64 \%$ \\
\end{tabular} & $\begin{array}{l}65 \% \\
\end{array}$ & \begin{tabular}{|l|l|}
$61 \%$ \\
\end{tabular} & \begin{tabular}{ll|}
$63 \%$ \\
\end{tabular} & \begin{tabular}{ll|}
$64 \%$ \\
\end{tabular} & $66 \%$ & \begin{tabular}{|l|l|}
$62 \%$ \\
\end{tabular} & \begin{tabular}{|l|}
$69 \%$ \\
\end{tabular} & $\begin{array}{l}62 \% \\
\end{array}$ \\
\hline Currency Subcategory & 1107 & 3509 & 4896 & 2744 & 2306 & 3269 & 3075 & 7869 & 4856 & 3836 & 1242 & 4469 & 3141 & 5424 & 6345 & 10365 & 1354 & 1070 & 1407 & 1886 & & 74170 \\
\hline Negative & $19 \%$ & $22 \%$ & $21 \%$ & $17 \%$ & $23 \%$ & $21 \%$ & $25 \%$ & $23 \%$ & $20 \%$ & $18 \%$ & $25 \%$ & $23 \%$ & $22 \%$ & $23 \%$ & $20 \%$ & $23 \%$ & $13 \%$ & $39 \%$ & $23 \%$ & $26 \%$ & & $22 \%$ \\
\hline Neutral & $18 \%$ & $17 \%$ & $17 \%$ & $16 \%$ & $16 \%$ & $18 \%$ & $21 \%$ & $19 \%$ & $18 \%$ & $17 \%$ & $16 \%$ & $15 \%$ & $14 \%$ & $12 \%$ & $13 \%$ & $14 \%$ & $11 \%$ & $11 \%$ & $10 \%$ & $9 \%$ & & $15 \%$ \\
\hline Positive & $63 \%$ & $61 \%$ & $61 \%$ & $67 \%$ & $61 \%$ & \begin{tabular}{|l|l|}
$61 \%$ & \\
\end{tabular} & $54 \%$ & $58 \%$ & $62 \%$ & $65 \%$ & $59 \%$ & $62 \%$ & $65 \%$ & $65 \%$ & $67 \%$ & $63 \%$ & $76 \%$ & $50 \%$ & $67 \%$ & $64 \%$ & & $63 \%$ \\
\hline Financial Subcategory & & 1431 & & & & & 1651 & 3297 & 3909 & 3079 & 3968 & 3692 & 1494 & & 4150 & 6809 & 2798 & 2376 & 1194 & 835 & & 40683 \\
\hline Negative & & $20 \%$ & & & & & $21 \%$ & $27 \%$ & $24 \%$ & $23 \%$ & $21 \%$ & $23 \%$ & $29 \%$ & & $26 \%$ & $25 \%$ & $27 \%$ & $22 \%$ & $14 \%$ & $23 \%$ & & $24 \%$ \\
\hline Neutral & & $19 \%$ & & & & & $20 \%$ & $20 \%$ & $21 \%$ & $14 \%$ & $15 \%$ & $16 \%$ & $12 \%$ & & $14 \%$ & $16 \%$ & $12 \%$ & $10 \%$ & $8 \%$ & $7 \%$ & & $15 \%$ \\
\hline Positive & & $61 \%$ & & & & & $60 \%$ & $53 \%$ & $56 \%$ & $62 \%$ & $64 \%$ & $61 \%$ & $59 \%$ & & $60 \%$ & $60 \%$ & $62 \%$ & $69 \%$ & $78 \%$ & $70 \%$ & & $61 \%$ \\
\hline Price Subcategory & & 2460 & 2096 & & & 1673 & & & & 4046 & & & & 1347 & 13140 & 8019 & 5760 & 2697 & 2735 & 2319 & 1190 & 47482 \\
\hline Negative & & $27 \%$ & $23 \%$ & & & $19 \%$ & & & & $27 \%$ & & & & $27 \%$ & $21 \%$ & $26 \%$ & $26 \%$ & $22 \%$ & $27 \%$ & $29 \%$ & $23 \%$ & $24 \%$ \\
\hline Neutral & & $22 \%$ & $18 \%$ & & & $21 \%$ & & & & $17 \%$ & & & & $13 \%$ & $13 \%$ & $13 \%$ & $13 \%$ & $12 \%$ & $12 \%$ & $13 \%$ & $8 \%$ & $14 \%$ \\
\hline Positive & & $52 \%$ & $59 \%$ & & & $59 \%$ & & & & $55 \%$ & & & & $60 \%$ & $66 \%$ & $61 \%$ & $61 \%$ & $66 \%$ & $61 \%$ & $57 \%$ & $69 \%$ & $61 \%$ \\
\hline Regulations & 321 & 1806 & 4325 & 918 & 728 & 1706 & 1545 & 7190 & 2200 & 3957 & & & & 2593 & 5963 & 5143 & 2438 & 1598 & 2759 & 2413 & 1634 & 49237 \\
\hline Negative & $31 \%$ & $36 \%$ & $25 \%$ & $37 \%$ & $29 \%$ & $37 \%$ & $34 \%$ & $34 \%$ & $42 \%$ & $35 \%$ & & & & $27 \%$ & $34 \%$ & $33 \%$ & $27 \%$ & $32 \%$ & $37 \%$ & $32 \%$ & $40 \%$ & $33 \%$ \\
\hline Neutral & $15 \%$ & $15 \%$ & $25 \%$ & $12 \%$ & $17 \%$ & $15 \%$ & $17 \%$ & $23 \%$ & $16 \%$ & $16 \%$ & & & & $11 \%$ & $13 \%$ & $13 \%$ & $12 \%$ & $10 \%$ & $9 \%$ & $9 \%$ & $10 \%$ & $16 \%$ \\
\hline Positive & $54 \%$ & $49 \%$ & $49 \%$ & $52 \%$ & $54 \%$ & $48 \%$ & $49 \%$ & $42 \%$ & $42 \%$ & $49 \%$ & & & & $62 \%$ & $53 \%$ & $54 \%$ & $61 \%$ & $58 \%$ & $54 \%$ & $59 \%$ & $50 \%$ & $51 \%$ \\
\hline Technical & 1001 & \begin{tabular}{|l|}
6058 \\
\end{tabular} & 3775 & 2591 & 2320 & 2958 & 4550 & 3414 & 4111 & \begin{tabular}{|l|l|}
4737 \\
\end{tabular} & 5649 & 4882 & 3280 & 6180 & \begin{tabular}{|l|}
13267 \\
\end{tabular} & 4181 & 4248 & 2588 & 1608 & 1149 & 1031 & 83578 \\
\hline Negative & $26 \%$ & $29 \%$ & $22 \%$ & $24 \%$ & $22 \%$ & $23 \%$ & $22 \%$ & $24 \%$ & $22 \%$ & $27 \%$ & $27 \%$ & $27 \%$ & $28 \%$ & $34 \%$ & $27 \%$ & $25 \%$ & $26 \%$ & $24 \%$ & $23 \%$ & $24 \%$ & $26 \%$ & $26 \%$ \\
\hline Neutral & $18 \%$ & $20 \%$ & $21 \%$ & $18 \%$ & $20 \%$ & $20 \%$ & $20 \%$ & $19 \%$ & $19 \%$ & $16 \%$ & $18 \%$ & $15 \%$ & $13 \%$ & $12 \%$ & $13 \%$ & $13 \%$ & $13 \%$ & $13 \%$ & $11 \%$ & $11 \%$ & $10 \%$ & $16 \%$ \\
\hline Positive & $56 \%$ & $52 \%$ & $57 \%$ & $57 \%$ & $58 \%$ & $57 \%$ & $58 \%$ & $56 \%$ & $59 \%$ & $57 \%$ & $55 \%$ & $58 \%$ & $59 \%$ & $54 \%$ & $60 \%$ & $62 \%$ & $60 \%$ & $63 \%$ & $66 \%$ & $65 \%$ & $65 \%$ & $58 \%$ \\
\hline Others Subcategory & 603 & & & 638 & \begin{tabular}{|l|}
520 \\
\end{tabular} & & \begin{tabular}{|l|l|}
1362 \\
\end{tabular} & & \begin{tabular}{|l|}
1899 \\
\end{tabular} & \begin{tabular}{|l|}
2876 \\
\end{tabular} & 3865 & \begin{tabular}{|l|l|}
2856 \\
\end{tabular} & 1872 & \begin{tabular}{|l|l|}
4196 \\
\end{tabular} & \begin{tabular}{|l|l|}
4539 \\
\end{tabular} & & 2577 & & & & 415 & \begin{tabular}{|l|l|}
28218 \\
\end{tabular} \\
\hline Negative & $27 \%$ & & & $28 \%$ & $27 \%$ & & $20 \%$ & & $21 \%$ & $28 \%$ & $30 \%$ & $31 \%$ & $34 \%$ & $39 \%$ & $31 \%$ & & $25 \%$ & & & & $22 \%$ & $30 \%$ \\
\hline Neutral & $18 \%$ & & & $15 \%$ & \begin{tabular}{|l|}
$17 \%$ \\
\end{tabular} & & $19 \%$ & & $20 \%$ & \begin{tabular}{|l|}
$16 \%$ \\
\end{tabular} & \begin{tabular}{|l|}
$19 \%$ \\
\end{tabular} & $15 \%$ & $13 \%$ & $12 \%$ & $\begin{array}{l}13 \% \\
\end{array}$ & & $13 \%$ & & & & $8 \%$ & $15 \%$ \\
\hline Positive & $55 \%$ & & & $57 \%$ & $56 \%$ & & $60 \%$ & & $58 \%$ & $56 \%$ & $50 \%$ & $54 \%$ & $53 \%$ & $49 \%$ & $56 \%$ & & $62 \%$ & & & & $70 \%$ & $55 \%$ \\
\hline Wallet Subcategory & 398 & 6058 & 3775 & 1953 & 1800 & 2958 & 3188 & 3414 & 2212 & 1861 & 1784 & 2026 & 1408 & 1984 & 8728 & 4181 & 1671 & 2588 & 1608 & 1149 & 616 & 55360 \\
\hline Negative & $24 \%$ & $29 \%$ & $22 \%$ & $23 \%$ & $21 \%$ & $23 \%$ & $23 \%$ & $24 \%$ & $23 \%$ & $27 \%$ & $20 \%$ & $22 \%$ & $20 \%$ & $22 \%$ & $24 \%$ & $25 \%$ & $27 \%$ & $24 \%$ & $23 \%$ & $24 \%$ & $28 \%$ & $24 \%$ \\
\hline Neutral & $18 \%$ & $20 \%$ & $21 \%$ & $20 \%$ & $20 \%$ & $20 \%$ & $20 \%$ & $\begin{array}{l}19 \% \\
\end{array}$ & \begin{tabular}{|l|}
$18 \%$ \\
\end{tabular} & $16 \%$ & $\begin{array}{l}16 \% \\
\end{array}$ & $15 \%$ & $\begin{array}{l}13 \% \\
\end{array}$ & \begin{tabular}{ll|}
$12 \%$ \\
\end{tabular} & \begin{tabular}{|l|}
$13 \%$ \\
\end{tabular} & $\begin{array}{l}13 \% \\
\end{array}$ & $\begin{array}{l}15 \% \\
\end{array}$ & $\begin{array}{l}13 \% \\
\end{array}$ & $11 \%$ & \begin{tabular}{|l|l|}
$11 \%$ \\
\end{tabular} & $\begin{array}{l}11 \% \\
\end{array}$ & $\begin{array}{l}16 \% \\
\end{array}$ \\
\hline Positive & $58 \%$ & $52 \%$ & $57 \%$ & $57 \%$ & $59 \%$ & $57 \%$ & $57 \%$ & $56 \%$ & $59 \%$ & $57 \%$ & $64 \%$ & $63 \%$ & $66 \%$ & $66 \%$ & $63 \%$ & $62 \%$ & $58 \%$ & $63 \%$ & $66 \%$ & $65 \%$ & $62 \%$ & $60 \%$ \\
\hline Off Topic & \begin{tabular}{|l|l|}
1147 \\
\end{tabular} & \begin{tabular}{|l|l|}
5826 \\
\end{tabular} & 6777 & 3315 & \begin{tabular}{|l|l}
1761 \\
\end{tabular} & \begin{tabular}{|l|}
5332 \\
\end{tabular} & \begin{tabular}{|l|l|}
4868 \\
\end{tabular} & \begin{tabular}{|l|}
11347 \\
\end{tabular} & 6702 & \begin{tabular}{|l|}
3024 \\
\end{tabular} & 4028 & \begin{tabular}{|l|l|}
4271 \\
\end{tabular} & 2252 & 3746 & \begin{tabular}{|l|l|}
7508 \\
\end{tabular} & 3021 & \begin{tabular}{|l|l|}
1707 \\
\end{tabular} & 1082 & \begin{tabular}{|l|}
2109 \\
\end{tabular} & & \begin{tabular}{|l|}
497 \\
\end{tabular} & \begin{tabular}{|l|}
80320 \\
\end{tabular} \\
\hline Negative & $15 \%$ & $\begin{array}{l}19 \% \\
\end{array}$ & $21 \%$ & $17 \%$ & $26 \%$ & $18 \%$ & $18 \%$ & $19 \%$ & $20 \%$ & $21 \%$ & $\begin{array}{l}19 \% \\
\end{array}$ & $20 \%$ & $21 \%$ & $33 \%$ & $27 \%$ & $20 \%$ & $30 \%$ & $18 \%$ & $20 \%$ & & $16 \%$ & $21 \%$ \\
\hline Neutral & $20 \%$ & $27 \%$ & $\begin{array}{l}18 \% \\
\end{array}$ & \begin{tabular}{|l|l|}
$19 \%$ \\
\end{tabular} & $\begin{array}{l}16 \% \\
\end{array}$ & $20 \%$ & $17 \%$ & $\begin{array}{l}19 \% \\
\end{array}$ & \begin{tabular}{|l|}
$17 \%$ \\
\end{tabular} & $25 \%$ & \begin{tabular}{|l|}
$14 \%$ \\
\end{tabular} & \begin{tabular}{|l|}
$14 \%$ \\
\end{tabular} & \begin{tabular}{ll|}
$11 \%$ \\
\end{tabular} & $11 \%$ & \begin{tabular}{|l|}
$12 \%$ \\
\end{tabular} & $\begin{array}{l}13 \% \\
\end{array}$ & $12 \%$ & $8 \%$ & $9 \%$ & & \begin{tabular}{|l|l|}
$11 \%$ \\
\end{tabular} & $\begin{array}{l}17 \% \\
\end{array}$ \\
\hline Positive & $65 \%$ & \begin{tabular}{|l|l}
$54 \%$ \\
\end{tabular} & $\begin{array}{ll}61 \% \\
\end{array}$ & \begin{tabular}{ll|}
$64 \%$ \\
\end{tabular} & \begin{tabular}{|l|l|}
$58 \%$ \\
\end{tabular} & \begin{tabular}{|l|}
$62 \%$ \\
\end{tabular} & \begin{tabular}{|l|l|}
$64 \%$ \\
\end{tabular} & \begin{tabular}{|l|}
$62 \%$ \\
\end{tabular} & \begin{tabular}{|l|l|}
$64 \%$ \\
\end{tabular} & \begin{tabular}{|l|l|}
$54 \%$ \\
\end{tabular} & $\begin{array}{l}67 \% \\
\end{array}$ & $65 \%$ & $\begin{array}{l}68 \% \\
\end{array}$ & $56 \%$ & \begin{tabular}{|l|l|}
$61 \%$ \\
\end{tabular} & \begin{tabular}{|l|}
$67 \%$ \\
\end{tabular} & $58 \%$ & $74 \%$ & $71 \%$ & & \begin{tabular}{|l|}
$72 \%$ \\
\end{tabular} & $62 \%$ \\
\hline
\end{tabular}

Table 10: Distribution of positive/negative posts in Future category topics over the time.

\begin{tabular}{|c|c|c|c|c|c|c|c|c|c|c|c|c|c|c|c|c|c|c|c|c|c|c|}
\hline Category/Topic & $2010 \mathrm{~b}$ & 2011a & $2011 \mathrm{~b}$ & $2012 \mathrm{a}$ & $2012 \mathrm{~b}$ & 2013a & $2013 \mathrm{~b}$ & 2014a & $2014 \mathrm{~b}$ & $2015 \mathrm{a}$ & $2015 \mathrm{~b}$ & $2016 \mathrm{a}$ & $2016 \mathrm{~b}$ & $2017 \mathrm{a}$ & $2017 \mathrm{~b}$ & $2018 \mathrm{a}$ & $2018 \mathrm{~b}$ & $2019 \mathrm{a}$ & $2019 \mathrm{~b}$ & $2020 \mathrm{a}$ & $2020 \mathrm{~b}$ & Total \\
\hline \# Posts & & 3361 & 1218 & 933 & 1350 & & 1180 & 2218 & 1489 & & 2256 & & 1143 & 2014 & & 2500 & 1582 & 970 & 889 & 879 & 1343 & 25325 \\
\hline Cryptocurrency Adoption & & & & & & & & & & & & & & & & & & 970 & 889 & & 440 & 2299 \\
\hline Negative & & & & & & & & & & & & & & & & & & $19 \%$ & $18 \%$ & & $19 \%$ & $18 \%$ \\
\hline Neutral & & & & & & & & & & & & & & & & & & $14 \%$ & $8 \%$ & & $9 \%$ & $11 \%$ \\
\hline Positive & & & & & & & & & & & & & & & & & & $67 \%$ & $74 \%$ & & $72 \%$ & $71 \%$ \\
\hline Future of Bitcoin & & 1676 & & & 706 & & 1180 & & & & & & & 952 & & & & & & & 355 & 4869 \\
\hline Negative & & $24 \%$ & & & $26 \%$ & & $23 \%$ & & & & & & & $26 \%$ & & & & & & & $30 \%$ & $25 \%$ \\
\hline Neutral & & $25 \%$ & & & $16 \%$ & & $21 \%$ & & & & & & & $16 \%$ & & & & & & & $12 \%$ & $20 \%$ \\
\hline Positive & & $51 \%$ & & & $58 \%$ & & $56 \%$ & & & & & & & $58 \%$ & & & & & & & $59 \%$ & $55 \%$ \\
\hline Future Role of Bitcoin & & 1685 & 1218 & 933 & 644 & & & 2218 & 1489 & & 2256 & & 1143 & 1062 & & 2500 & 1582 & & & 879 & 548 & 18157 \\
\hline Negative & & $28 \%$ & $24 \%$ & $19 \%$ & $20 \%$ & & & $22 \%$ & $23 \%$ & & $34 \%$ & & $21 \%$ & $21 \%$ & & $12 \%$ & $14 \%$ & & & $16 \%$ & $17 \%$ & $21 \%$ \\
\hline Neutral & & $17 \%$ & $15 \%$ & $15 \%$ & $19 \%$ & & & $17 \%$ & $18 \%$ & & $15 \%$ & & $20 \%$ & $11 \%$ & & $10 \%$ & $9 \%$ & & & $8 \%$ & $14 \%$ & $14 \%$ \\
\hline Positive & & $55 \%$ & $61 \%$ & $66 \%$ & $61 \%$ & & & $61 \%$ & $59 \%$ & & $51 \%$ & & $59 \%$ & $68 \%$ & & $78 \%$ & $77 \%$ & & & $77 \%$ & $69 \%$ & $64 \%$ \\
\hline
\end{tabular}


Table 11: Distribution of positive/negative posts in General category topics over the time.

\begin{tabular}{|c|c|c|c|c|c|c|c|c|c|c|c|c|c|c|c|c|c|c|c|c|c|c|}
\hline Category/Topic & $2010 \mathrm{~b}$ & 2011a & $2011 \mathrm{~b}$ & $2012 \mathrm{a}$ & $2012 \mathrm{~b}$ & $2013 \mathrm{a}$ & $2013 \mathrm{~b}$ & 2014a & $2014 \mathrm{~b}$ & $2015 \mathrm{a}$ & $2015 \mathrm{~b}$ & $2016 \mathrm{a}$ & $2016 \mathrm{~b}$ & $2017 \mathrm{a}$ & $2017 \mathrm{~b}$ & $2018 \mathrm{a}$ & $2018 \mathrm{~b}$ & $2019 \mathrm{a}$ & $2019 \mathrm{~b}$ & $2020 \mathrm{a}$ & $2020 \mathrm{~b}$ & Total \\
\hline \# Posts & 627 & 4205 & 2417 & 1290 & 2191 & 4501 & 4448 & 5314 & 6496 & 3563 & 5454 & 4147 & 3503 & 7240 & 6343 & 10235 & 2715 & 4777 & 4520 & 4171 & 1673 & 89830 \\
\hline Bitcoin Transaction & & & & & & 1131 & & & 1842 & 1723 & & 1433 & 1347 & 1940 & & 2867 & 1157 & 774 & 1122 & 910 & & 16246 \\
\hline Negative & & & & & & $24 \%$ & & & $26 \%$ & $30 \%$ & & $25 \%$ & $29 \%$ & $24 \%$ & & $29 \%$ & $25 \%$ & $15 \%$ & $28 \%$ & $16 \%$ & & $26 \%$ \\
\hline Neutral & & & & & & $19 \%$ & & & $23 \%$ & $18 \%$ & & $18 \%$ & $13 \%$ & $14 \%$ & & $14 \%$ & $14 \%$ & $8 \%$ & $10 \%$ & $9 \%$ & & $15 \%$ \\
\hline Positive & & & & & & $57 \%$ & & & $51 \%$ & $52 \%$ & & $57 \%$ & $59 \%$ & $62 \%$ & & $57 \%$ & $61 \%$ & $76 \%$ & $62 \%$ & $74 \%$ & & $59 \%$ \\
\hline Bitcoin Foundation & & & & & & 1340 & & 2147 & 1737 & & & & & & & & & & & & & 5224 \\
\hline Negative & & & & & & $18 \%$ & & $18 \%$ & $17 \%$ & & & & & & & & & & & & & $17 \%$ \\
\hline Neutral & & & & & & $16 \%$ & & $16 \%$ & $17 \%$ & & & & & & & & & & & & & $16 \%$ \\
\hline Positive & & & & & & $66 \%$ & & $67 \%$ & $66 \%$ & & & & & & & & & & & & & $66 \%$ \\
\hline Bitcoin Transaction Cost/Time & 277 & 1690 & & & & & 1545 & & & & & & & 2881 & & & & 1050 & & 1070 & 670 & 9183 \\
\hline Negative & $19 \%$ & $40 \%$ & & & & & $23 \%$ & & & & & & & $37 \%$ & & & & $26 \%$ & & $20 \%$ & $27 \%$ & $31 \%$ \\
\hline Neutral & $15 \%$ & $18 \%$ & & & & & $16 \%$ & & & & & & & $14 \%$ & & & & $13 \%$ & & $10 \%$ & $13 \%$ & $14 \%$ \\
\hline Positive & $65 \%$ & $42 \%$ & & & & & $61 \%$ & & & & & & & $49 \%$ & & & & $61 \%$ & & $69 \%$ & $61 \%$ & $55 \%$ \\
\hline Bitcoin User Experience & & & & & & 2030 & & & & & & & & & & & & & & & & 2030 \\
\hline Negative & & & & & & $14 \%$ & & & & & & & & & & & & & & & & $14 \%$ \\
\hline Neutral & & & & & & $33 \%$ & & & & & & & & & & & & & & & & $33 \%$ \\
\hline Positive & & & & & & $53 \%$ & & & & & & & & & & & & & & & & $53 \%$ \\
\hline Blockchain Technology & & & & & & & & & & & 1599 & & 972 & & 3318 & 3399 & 1558 & 1262 & 895 & & 518 & 13521 \\
\hline Negative & & & & & & & & & & & $19 \%$ & & $23 \%$ & & $14 \%$ & $13 \%$ & $12 \%$ & $12 \%$ & $20 \%$ & & $13 \%$ & $15 \%$ \\
\hline Neutral & & & & & & & & & & & $14 \%$ & & $11 \%$ & & $10 \%$ & $11 \%$ & $11 \%$ & $11 \%$ & $10 \%$ & & $7 \%$ & $11 \%$ \\
\hline Positive & & & & & & & & & & & $67 \%$ & & $66 \%$ & & $76 \%$ & $76 \%$ & $76 \%$ & $77 \%$ & $71 \%$ & & $80 \%$ & $74 \%$ \\
\hline Effects of Covid on Bitcoin & & & & & & & & & & & & & & & & & & & & 847 & & 847 \\
\hline Negative & & & & & & & & & & & & & & & & & & & & $30 \%$ & & $30 \%$ \\
\hline Neutral & & & & & & & & & & & & & & & & & & & & $10 \%$ & & $10 \%$ \\
\hline Positive & & & & & & & & & & & & & & & & & & & & $60 \%$ & & $60 \%$ \\
\hline General Bitcoin Discussion & & & & & 655 & & 996 & & 2917 & 1840 & 1796 & 1281 & & & 3025 & & & 1691 & 1338 & 1344 & 485 & 17368 \\
\hline Negative & & & & & $20 \%$ & & $26 \%$ & & $18 \%$ & $14 \%$ & $27 \%$ & $35 \%$ & & & $27 \%$ & & & $23 \%$ & $29 \%$ & $35 \%$ & $28 \%$ & $25 \%$ \\
\hline Neutral & & & & & $17 \%$ & & $18 \%$ & & $30 \%$ & $15 \%$ & $18 \%$ & $18 \%$ & & & $15 \%$ & & & $10 \%$ & $13 \%$ & $10 \%$ & $14 \%$ & $17 \%$ \\
\hline Positive & & & & & $63 \%$ & & $56 \%$ & & $52 \%$ & $71 \%$ & $55 \%$ & $47 \%$ & & & $58 \%$ & & & $67 \%$ & $58 \%$ & $55 \%$ & $57 \%$ & $58 \%$ \\
\hline Project Development & 350 & 2515 & 2417 & 1290 & 1536 & & 1907 & 3167 & & & 2059 & 1433 & 1184 & 2419 & & 3969 & & & 1165 & & & 25411 \\
\hline Negative & $14 \%$ & $18 \%$ & $15 \%$ & $12 \%$ & $14 \%$ & & $20 \%$ & $30 \%$ & & & $23 \%$ & $27 \%$ & $15 \%$ & $14 \%$ & & $35 \%$ & & & $22 \%$ & & & $22 \%$ \\
\hline Neutral & $16 \%$ & $24 \%$ & $21 \%$ & $20 \%$ & $26 \%$ & & $20 \%$ & $17 \%$ & & & $13 \%$ & $15 \%$ & $11 \%$ & $13 \%$ & & $12 \%$ & & & $13 \%$ & & & $17 \%$ \\
\hline Positive & $70 \%$ & $58 \%$ & $63 \%$ & $69 \%$ & $59 \%$ & & $60 \%$ & $52 \%$ & & & $64 \%$ & $58 \%$ & $74 \%$ & $73 \%$ & & $53 \%$ & & & \begin{tabular}{|l|l}
$65 \%$ \\
\end{tabular} & & & $61 \%$ \\
\hline
\end{tabular}

Table 12: Distribution of positive/negative posts in Mining category topics over the time.

\begin{tabular}{|l|l|l|l|l|l|l|l|l|l|l|l|l|l|l|l|l|l|l|l|l|l|l|}
\hline Category/Topic & $2010 \mathrm{~b}$ & $2011 \mathrm{a}$ & $2011 \mathrm{~b}$ & $2012 \mathrm{a}$ & $2012 \mathrm{~b}$ & $2013 \mathrm{a}$ & $2013 \mathrm{~b}$ & $2014 \mathrm{a}$ & $2014 \mathrm{~b}$ & $2015 \mathrm{a}$ & $2015 \mathrm{~b}$ & $2016 \mathrm{a}$ & $2016 \mathrm{~b}$ & $2017 \mathrm{a}$ & $2017 \mathrm{~b}$ & $2018 \mathrm{a}$ & $2018 \mathrm{~b}$ & $2019 \mathrm{a}$ & $2019 \mathrm{~b}$ & $2020 \mathrm{a}$ & $2020 \mathrm{~b}$ & Total \\
\hline \# Posts & 1010 & 2088 & 1837 & 2245 & 1476 & 4305 & 2492 & 2987 & 1861 & 2159 & 4643 & 3791 & 2470 & 1561 & 6967 & 4633 & 1492 & 1053 & 1294 & 1807 & 567 & 52738 \\
\hline Mining : Transactions & 341 & & 1837 & 1393 & 843 & 1701 & 2492 & 2987 & 1861 & 2159 & 2192 & 2606 & 1134 & 1561 & 6967 & 4633 & & & & 734 & 567 & 36008 \\
\hline Negative & $52 \%$ & & $35 \%$ & $28 \%$ & $27 \%$ & $38 \%$ & $27 \%$ & $36 \%$ & $36 \%$ & $36 \%$ & $44 \%$ & $36 \%$ & $31 \%$ & $35 \%$ & $32 \%$ & $30 \%$ & & & & $22 \%$ & $33 \%$ & $33 \%$ \\
\hline Neutral & $14 \%$ & & $17 \%$ & $31 \%$ & $21 \%$ & $17 \%$ & $32 \%$ & $19 \%$ & $17 \%$ & $16 \%$ & $15 \%$ & $21 \%$ & $16 \%$ & $12 \%$ & $18 \%$ & $12 \%$ & & & & $14 \%$ & $9 \%$ & $18 \%$ \\
\hline Positive & $34 \%$ & & $48 \%$ & $41 \%$ & $52 \%$ & $46 \%$ & $41 \%$ & $45 \%$ & $47 \%$ & $48 \%$ & $41 \%$ & $42 \%$ & $54 \%$ & $52 \%$ & $49 \%$ & $58 \%$ & & & & $63 \%$ & $58 \%$ & $48 \%$ \\
\hline Mining: Requirement & 669 & 2088 & & 852 & 633 & 2604 & & & & & 2451 & 1185 & 1336 & & & & 1492 & 1053 & 1294 & 1073 & & 16730 \\
\hline Negative & $23 \%$ & $34 \%$ & & $24 \%$ & $24 \%$ & $24 \%$ & & & & & $28 \%$ & $24 \%$ & $21 \%$ & & & & $29 \%$ & $33 \%$ & $31 \%$ & $32 \%$ & & $28 \%$ \\
\hline Neutral & $18 \%$ & $18 \%$ & & $19 \%$ & $21 \%$ & $22 \%$ & & & & & $18 \%$ & $15 \%$ & $14 \%$ & & & & $10 \%$ & $11 \%$ & $11 \%$ & $8 \%$ & & $16 \%$ \\
\hline Positive & $58 \%$ & $48 \%$ & & $57 \%$ & $55 \%$ & $54 \%$ & & & & & $55 \%$ & $61 \%$ & $65 \%$ & & & & $61 \%$ & $56 \%$ & $58 \%$ & $59 \%$ & & $56 \%$ \\
\hline
\end{tabular}


Table 13: Distribution of positive/negative posts in Monetary category topics over the time.

\begin{tabular}{|c|c|c|c|c|c|c|c|c|c|c|c|c|c|c|c|c|c|c|c|c|c|c|}
\hline Category/Topic & $2010 \mathrm{~b}$ & 2011a & \begin{tabular}{|l|l|}
$2011 \mathrm{~b}$ \\
\end{tabular} & 2012a & 2012b & $2013 \mathrm{a}$ & $2013 \mathrm{~b}$ & $2014 a$ & $2014 \mathrm{~b}$ & $2015 \mathrm{a}$ & $2015 \mathrm{~b}$ & $2016 \mathrm{a}$ & \begin{tabular}{|l|l|}
$2016 \mathrm{~b}$ \\
\end{tabular} & $2017 \mathrm{a}$ & $2017 \mathrm{~b}$ & $2018 \mathrm{a}$ & \begin{tabular}{|l|l|}
$2018 \mathrm{~b}$ \\
\end{tabular} & $2019 a$ & 2019b & $2020 \mathrm{a}$ & $2020 \mathrm{~b}$ & \begin{tabular}{|l|} 
Total \\
\end{tabular} \\
\hline \# Posts & 1107 & 7400 & 6992 & 2744 & 2306 & 4942 & 4726 & 11166 & 8765 & 10961 & 5210 & 8161 & 4635 & 6771 & 23635 & 25193 & 9912 & 6143 & 5336 & 5040 & 1190 & 162335 \\
\hline Currency Subcategory & 1107 & 3509 & 4896 & 2744 & 2306 & 3269 & 3075 & 7869 & 4856 & 3836 & 1242 & 4469 & 3141 & 5424 & 6345 & 10365 & 1354 & 1070 & 1407 & 1886 & 0 & 74170 \\
\hline Bitcoin Alternatives & 287 & & & & & & & 2104 & & & & & 1882 & 3068 & 2732 & 3321 & & 1070 & & 859 & & 15323 \\
\hline Negative & $26 \%$ & & & & & & & $27 \%$ & & & & & $26 \%$ & $25 \%$ & $20 \%$ & $29 \%$ & & $39 \%$ & & $27 \%$ & & $26 \%$ \\
\hline Neutral & $18 \%$ & & & & & & & $24 \%$ & & & & & $14 \%$ & $12 \%$ & $12 \%$ & $13 \%$ & & $11 \%$ & & $11 \%$ & & $14 \%$ \\
\hline Positive & $56 \%$ & & & & & & & $49 \%$ & & & & & $60 \%$ & $63 \%$ & $68 \%$ & $59 \%$ & & $50 \%$ & & $61 \%$ & & $60 \%$ \\
\hline Bitcoin Exchange Rate & 444 & 1786 & 1511 & 964 & 887 & 1615 & 1580 & 2720 & 2385 & & 1242 & 2343 & & & 3613 & 3288 & 1354 & & 1407 & 1027 & & 28166 \\
\hline Negative & $16 \%$ & $24 \%$ & $18 \%$ & $16 \%$ & $24 \%$ & $21 \%$ & $20 \%$ & $28 \%$ & $25 \%$ & & $25 \%$ & $29 \%$ & & & $20 \%$ & $20 \%$ & $13 \%$ & & $23 \%$ & $26 \%$ & & $22 \%$ \\
\hline Neutral & $27 \%$ & $16 \%$ & $17 \%$ & $16 \%$ & $15 \%$ & $19 \%$ & $21 \%$ & $16 \%$ & $19 \%$ & & $16 \%$ & $15 \%$ & & & $14 \%$ & $13 \%$ & $11 \%$ & & $10 \%$ & $8 \%$ & & $15 \%$ \\
\hline Positive & $57 \%$ & $60 \%$ & $65 \%$ & $68 \%$ & $61 \%$ & $60 \%$ & $59 \%$ & $55 \%$ & $56 \%$ & & $59 \%$ & $56 \%$ & & & $65 \%$ & $67 \%$ & $76 \%$ & & $67 \%$ & $67 \%$ & & $62 \%$ \\
\hline Payment with Bitcoin & 376 & 1723 & 3385 & 1780 & 1419 & 1654 & 1495 & 3045 & 2471 & 3836 & & 2126 & 1259 & 2356 & & 3756 & & & & & & 30681 \\
\hline Negative & $16 \%$ & $20 \%$ & $23 \%$ & $18 \%$ & $22 \%$ & $20 \%$ & $31 \%$ & $17 \%$ & $15 \%$ & $18 \%$ & & $16 \%$ & $16 \%$ & $20 \%$ & & $20 \%$ & & & & & & $19 \%$ \\
\hline Neutral & $9 \%$ & $18 \%$ & $17 \%$ & $16 \%$ & $16 \%$ & $17 \%$ & $20 \%$ & $17 \%$ & $16 \%$ & $17 \%$ & & $15 \%$ & $13 \%$ & $12 \%$ & & $17 \%$ & & & & & & $16 \%$ \\
\hline Positive & $75 \%$ & $62 \%$ & $60 \%$ & $66 \%$ & $62 \%$ & $62 \%$ & $49 \%$ & $67 \%$ & $68 \%$ & $65 \%$ & & $69 \%$ & $71 \%$ & $68 \%$ & & $63 \%$ & & & & & & $64 \%$ \\
\hline Financial Subcategory & & 1431 & & & & & 1651 & 3297 & 3909 & 3079 & 3968 & 3692 & 1494 & & 4150 & 6809 & 2798 & 2376 & 1194 & 835 & & 40683 \\
\hline Bitcoin Trading & & & & & & & & & & & & & & & & & & 1148 & 1194 & 835 & & 3177 \\
\hline Negative & & & & & & & & & & & & & & & & & & $19 \%$ & $14 \%$ & $23 \%$ & & $18 \%$ \\
\hline Neutral & & & & & & & & & & & & & & & & & & $8 \%$ & $8 \%$ & $7 \%$ & & $8 \%$ \\
\hline Positive & & & & & & & & & & & & & & & & & & $73 \%$ & $78 \%$ & $70 \%$ & & $74 \%$ \\
\hline Effect of Bitcoin on Banking & & & & & & & & & 1767 & 1464 & 1950 & 1748 & 1494 & & & 2983 & 1355 & 1228 & & & & 13989 \\
\hline Negative & & & & & & & & & $29 \%$ & $28 \%$ & $18 \%$ & $22 \%$ & $29 \%$ & & & $28 \%$ & $26 \%$ & $24 \%$ & & & & $25 \%$ \\
\hline Neutral & & & & & & & & & $18 \%$ & $14 \%$ & $15 \%$ & $16 \%$ & $12 \%$ & & & $15 \%$ & $13 \%$ & $11 \%$ & & & & $14 \%$ \\
\hline Positive & & & & & & & & & $53 \%$ & $59 \%$ & $67 \%$ & $62 \%$ & $59 \%$ & & & $57 \%$ & $61 \%$ & $65 \%$ & & & & $60 \%$ \\
\hline Financial Return & & 1431 & & & & & 1651 & 3297 & 2142 & 1615 & 2018 & 1944 & & & 4150 & 3826 & 1443 & & & & & 23517 \\
\hline Negative & & $20 \%$ & & & & & $21 \%$ & $27 \%$ & $20 \%$ & $20 \%$ & $23 \%$ & $23 \%$ & & & $26 \%$ & $22 \%$ & $27 \%$ & & & & & $24 \%$ \\
\hline Neutral & & $19 \%$ & & & & & $20 \%$ & $20 \%$ & $23 \%$ & $15 \%$ & $16 \%$ & $16 \%$ & & & $14 \%$ & $16 \%$ & $10 \%$ & & & & & $17 \%$ \\
\hline Positive & & $61 \%$ & & & & & $60 \%$ & $53 \%$ & $57 \%$ & $65 \%$ & $60 \%$ & $60 \%$ & & & $60 \%$ & $62 \%$ & $62 \%$ & & & & & $60 \%$ \\
\hline Price Subcategory & & 2460 & 2096 & & & 1673 & & & & 4046 & & & & 1347 & \begin{tabular}{|c|c|c|}
13140 & \\
\end{tabular} & 8019 & 5760 & 2697 & 2735 & 2319 & 1190 & 47482 \\
\hline Bitcoin Future Price Discussion & & & & & & & & & & 1592 & & & & 1347 & 3619 & 2665 & 1259 & 822 & 757 & 734 & 454 & 13249 \\
\hline Negative & & & & & & & & & & $24 \%$ & & & & $27 \%$ & $16 \%$ & $22 \%$ & $22 \%$ & $18 \%$ & $29 \%$ & $26 \%$ & $22 \%$ & $22 \%$ \\
\hline Neutral & & & & & & & & & & $20 \%$ & & & & $13 \%$ & $11 \%$ & $14 \%$ & $16 \%$ & $13 \%$ & $12 \%$ & $16 \%$ & $6 \%$ & $14 \%$ \\
\hline Positive & & & & & & & & & & $56 \%$ & & & & $60 \%$ & $73 \%$ & $63 \%$ & $62 \%$ & $69 \%$ & $59 \%$ & $58 \%$ & $72 \%$ & $65 \%$ \\
\hline Bitcoin Price Discussion & & 2460 & 2096 & & & 1673 & & & & 2454 & & & & & 9521 & 5354 & 4501 & 1875 & 1978 & 1585 & & 33497 \\
\hline Negative & & $27 \%$ & $23 \%$ & & & $19 \%$ & & & & $29 \%$ & & & & & $23 \%$ & $27 \%$ & $28 \%$ & $24 \%$ & $27 \%$ & $31 \%$ & & $26 \%$ \\
\hline Neutral & & $22 \%$ & $18 \%$ & & & $21 \%$ & & & & $16 \%$ & & & & & $14 \%$ & $13 \%$ & $12 \%$ & $11 \%$ & $12 \%$ & $13 \%$ & & $15 \%$ \\
\hline Positive & & $52 \%$ & $59 \%$ & & & $59 \%$ & & & & $55 \%$ & & & & & $63 \%$ & $60 \%$ & $60 \%$ & $65 \%$ & $61 \%$ & $57 \%$ & & $60 \%$ \\
\hline Bitcoin Price Discussion (Covid) & & & & & & & & & & & & & & & & & & & & & 736 & 736 \\
\hline Negative & & & & & & & & & & & & & & & & & & & & & $24 \%$ & $24 \%$ \\
\hline Neutral & & & & & & & & & & & & & & & & & & & & & $9 \%$ & $9 \%$ \\
\hline Positive & & & & & & & & & & & & & & & & & & & & & $67 \%$ & $67 \%$ \\
\hline
\end{tabular}

Table 14: Distribution of positive/negative posts in Regulations category topics over the time.

\begin{tabular}{|l|l|l|l|l|l|l|l|l|l|l|l|l|l|l|l|l|l|l|l|l|l|l|}
\hline Category/Topic & $2010 \mathrm{~b}$ & $2011 \mathrm{a}$ & $2011 \mathrm{~b}$ & $2012 \mathrm{a}$ & $2012 \mathrm{~b}$ & $2013 \mathrm{a}$ & $2013 \mathrm{~b}$ & $2014 \mathrm{a}$ & $2014 \mathrm{~b}$ & $2015 \mathrm{a}$ & $2015 \mathrm{~b}$ & $2016 \mathrm{a}$ & $2016 \mathrm{~b}$ & $2017 \mathrm{a}$ & $2017 \mathrm{~b}$ & $2018 \mathrm{a}$ & $2018 \mathrm{~b}$ & $2019 \mathrm{a}$ & $2019 \mathrm{~b}$ & $2020 \mathrm{a}$ & $2020 \mathrm{~b}$ & Total \\
\hline \# Posts & 321 & 1806 & 4325 & 918 & 728 & 1706 & 1545 & 7190 & 2200 & 3957 & & & & 2593 & 5963 & 5143 & 2438 & 1598 & 2759 & 2413 & 1634 & 49237 \\
\hline Bitcoin in Illegal Activity & & & 1693 & & & & & 4397 & & 2339 & & & & & & & & & & & 407 & 8836 \\
\hline Negative & & & $29 \%$ & & & & & $35 \%$ & & $37 \%$ & & & & & & & & & & & $43 \%$ & $35 \%$ \\
\hline Neutral & & & $19 \%$ & & & & & $28 \%$ & & $16 \%$ & & & & & & & & & & & $9 \%$ & $22 \%$ \\
\hline Positive & & & $52 \%$ & & & & & $37 \%$ & & $48 \%$ & & & & & & & & & & & $48 \%$ & $43 \%$ \\
\hline Bitcoin Regulatory & 321 & 1806 & 2632 & 918 & 728 & 1706 & 1545 & 2793 & 2200 & 1618 & & & & 2593 & 5963 & 5143 & 2438 & 1598 & 1630 & 1177 & 606 & 37415 \\
\hline Negative & $31 \%$ & $36 \%$ & $23 \%$ & $37 \%$ & $29 \%$ & $37 \%$ & $34 \%$ & $34 \%$ & $42 \%$ & $33 \%$ & & & & $27 \%$ & $34 \%$ & $33 \%$ & $27 \%$ & $32 \%$ & $32 \%$ & $32 \%$ & $28 \%$ & $32 \%$ \\
\hline Neutral & $15 \%$ & $15 \%$ & $29 \%$ & $12 \%$ & $17 \%$ & $15 \%$ & $17 \%$ & $16 \%$ & $16 \%$ & $16 \%$ & & & & $11 \%$ & $13 \%$ & $13 \%$ & $12 \%$ & $10 \%$ & $9 \%$ & $10 \%$ & $9 \%$ & $14 \%$ \\
\hline Positive & $54 \%$ & $49 \%$ & $47 \%$ & $52 \%$ & $54 \%$ & $48 \%$ & $49 \%$ & $50 \%$ & $42 \%$ & $51 \%$ & & & & $62 \%$ & $53 \%$ & $54 \%$ & $61 \%$ & $58 \%$ & $59 \%$ & $58 \%$ & $63 \%$ & $53 \%$ \\
\hline Bitcoin Scam & & & & & & & & & & & & & & & & & & & 1129 & 1236 & 621 & 2986 \\
\hline Negative & & & & & & & & & & & & & & & & & & & $43 \%$ & $32 \%$ & $49 \%$ & $40 \%$ \\
\hline Neutral & & & & & & & & & & & & & & & & & & & $9 \%$ & $9 \%$ & $12 \%$ & $10 \%$ \\
\hline Positive & & & & & & & & & & & & & & & & & & & $47 \%$ & $59 \%$ & $38 \%$ & $50 \%$ \\
\hline
\end{tabular}


Table 15: Distribution of positive/negative posts in Technical category topics over the time.

\begin{tabular}{|c|c|c|c|c|c|c|c|c|c|c|c|c|c|c|c|c|c|c|c|c|c|c|}
\hline Category/Topic & $2010 \mathrm{~b}$ & 2011a & 2011b & $2012 \mathrm{a}$ & $2012 \mathrm{~b}$ & 2013a & $2013 \mathrm{~b}$ & 2014a & 2014b & $2015 \mathrm{a}$ & $2015 \mathrm{~b}$ & 2016a & $2016 \mathrm{~b}$ & $2017 \mathrm{a}$ & $2017 \mathrm{~b}$ & 2018a & $2018 \mathrm{~b}$ & 2019a & $2019 \mathrm{~b}$ & 2020a & \begin{tabular}{|l|l|}
$2020 \mathrm{~b}$ \\
\end{tabular} & Total \\
\hline \# Posts & 1001 & 6058 & 3775 & 2591 & 2320 & 2958 & 4550 & 3414 & 4111 & 4737 & 5649 & 4882 & 3280 & 6180 & 13267 & 4181 & 4248 & 2588 & 1608 & 1149 & 1031 & 83578 \\
\hline Others Subcategory & 603 & & & 638 & 520 & & 1362 & & 1899 & 2876 & 3865 & 2856 & 1872 & 4196 & 4539 & & 2577 & & & & 415 & 28218 \\
\hline Fork & & & & & & & & & & 1549 & 2542 & 1521 & 1872 & 4196 & 4539 & & 1418 & & & & & 17637 \\
\hline Negative & & & & & & & & & & $32 \%$ & $28 \%$ & $29 \%$ & $34 \%$ & $39 \%$ & $31 \%$ & & $29 \%$ & & & & & $33 \%$ \\
\hline Neutral & & & & & & & & & & $15 \%$ & $21 \%$ & $16 \%$ & $13 \%$ & $12 \%$ & $13 \%$ & & $12 \%$ & & & & & $14 \%$ \\
\hline Positive & & & & & & & & & & $53 \%$ & $51 \%$ & $55 \%$ & $53 \%$ & $49 \%$ & $56 \%$ & & $58 \%$ & & & & & $53 \%$ \\
\hline General Network Discussion & & & & & & & 1362 & & & 1327 & & & & & & & & & & & & 2689 \\
\hline Negative & & & & & & & $20 \%$ & & & $23 \%$ & & & & & & & & & & & & $21 \%$ \\
\hline Neutral & & & & & & & $19 \%$ & & & $17 \%$ & & & & & & & & & & & & $18 \%$ \\
\hline Positive & & & & & & & $60 \%$ & & & $60 \%$ & & & & & & & & & & & & $60 \%$ \\
\hline \begin{tabular}{|l|} 
Network Security \\
\end{tabular} & 292 & & & & 520 & & & & & & & & & & & & & & & & & 812 \\
\hline Negative & $27 \%$ & & & & $27 \%$ & & & & & & & & & & & & & & & & & $27 \%$ \\
\hline Neutral & $23 \%$ & & & & $17 \%$ & & & & & & & & & & & & & & & & & $19 \%$ \\
\hline Positive & $50 \%$ & & & & $56 \%$ & & & & & & & & & & & & & & & & & $53 \%$ \\
\hline Technical Issue & 311 & & & 638 & & & & & 1899 & & 1323 & 1335 & & & & & 1159 & & & & 415 & 7080 \\
\hline Negative & $26 \%$ & & & $28 \%$ & & & & & $21 \%$ & & $35 \%$ & $33 \%$ & & & & & $21 \%$ & & & & $22 \%$ & $27 \%$ \\
\hline Neutral & $14 \%$ & & & $15 \%$ & & & & & $20 \%$ & & $16 \%$ & $14 \%$ & & & & & $13 \%$ & & & & $8 \%$ & $16 \%$ \\
\hline Positive & $60 \%$ & & & $57 \%$ & & & & & $58 \%$ & & $49 \%$ & $53 \%$ & & & & & $66 \%$ & & & & $70 \%$ & $57 \%$ \\
\hline Wallet Subcategory & 398 & 6058 & 3775 & 1953 & 1800 & 2958 & 3188 & 3414 & 2212 & 1861 & 1784 & 2026 & 1408 & 1984 & 8728 & 4181 & 1671 & 2588 & 1608 & 1149 & 616 & 55360 \\
\hline User Security & 398 & 3661 & 2042 & 853 & 1800 & 2958 & 1807 & 3414 & 2212 & 1861 & 1784 & 2026 & 1408 & 1984 & 4781 & 4181 & 1671 & 1161 & 1608 & 1149 & & 42759 \\
\hline Negative & $24 \%$ & $25 \%$ & $22 \%$ & $24 \%$ & $21 \%$ & $23 \%$ & $20 \%$ & $24 \%$ & $23 \%$ & $27 \%$ & $20 \%$ & $22 \%$ & $20 \%$ & $22 \%$ & $23 \%$ & $25 \%$ & $27 \%$ & $22 \%$ & $23 \%$ & $24 \%$ & & $23 \%$ \\
\hline Neutral & $18 \%$ & $18 \%$ & $21 \%$ & $18 \%$ & $20 \%$ & $20 \%$ & $17 \%$ & $19 \%$ & $18 \%$ & $16 \%$ & $16 \%$ & $15 \%$ & $13 \%$ & $12 \%$ & $12 \%$ & $13 \%$ & $15 \%$ & $13 \%$ & $11 \%$ & $11 \%$ & & $16 \%$ \\
\hline Positive & $58 \%$ & $57 \%$ & $57 \%$ & $58 \%$ & $59 \%$ & $57 \%$ & $62 \%$ & $56 \%$ & $59 \%$ & $57 \%$ & $64 \%$ & $63 \%$ & $66 \%$ & $66 \%$ & $65 \%$ & $62 \%$ & $58 \%$ & $65 \%$ & $66 \%$ & $65 \%$ & & $61 \%$ \\
\hline Wallet Apps & & 2397 & 1733 & 1100 & & & 1381 & & & & & & & & 3947 & & & 1427 & & & 616 & 12601 \\
\hline Negative & & $34 \%$ & $22 \%$ & $23 \%$ & & & $26 \%$ & & & & & & & & $26 \%$ & & & $25 \%$ & & & $28 \%$ & $27 \%$ \\
\hline Neutral & & $22 \%$ & $21 \%$ & $21 \%$ & & & $23 \%$ & & & & & & & & $14 \%$ & & & $13 \%$ & & & $11 \%$ & $18 \%$ \\
\hline Positive & & $44 \%$ & $58 \%$ & $57 \%$ & & & $51 \%$ & & & & & & & & $60 \%$ & & & $62 \%$ & & & $62 \%$ & $56 \%$ \\
\hline
\end{tabular}

